\title{
Asymptotics for Hankel Determinants Associated to a Hermite Weight with a Varying Discontinuity
}

Christophe CHARLIER ${ }^{\dagger}$ and Alfredo DEAÑO ${ }^{\ddagger}$

$\dagger$ Department of Mathematics, KTH Royal Institute of Technology, Lindstedtsvägen 25, SE-114 28 Stockholm, Sweden

E-mail: cchar@kth.se

$\ddagger$ School of Mathematics, Statistics and Actuarial Science, University of Kent, Canterbury CT2 $7 F S$, UK

E-mail: A.Deano-Cabrera@kent.ac.uk

Received November 02, 2017, in final form February 27, 2018; Published online March 07, 2018 https://doi.org/10.3842/SIGMA.2018.018

\begin{abstract}
We study $n \times n$ Hankel determinants constructed with moments of a Hermite weight with a Fisher-Hartwig singularity on the real line. We consider the case when the singularity is in the bulk and is both of root-type and jump-type. We obtain large $n$ asymptotics for these Hankel determinants, and we observe a critical transition when the size of the jumps varies with $n$. These determinants arise in the thinning of the generalised Gaussian unitary ensembles and in the construction of special function solutions of the Painlevé IV equation.
\end{abstract}

Key words: asymptotic analysis; Riemann-Hilbert problems; Hankel determinants; random matrix theory; Painlevé equations

2010 Mathematics Subject Classification: 30E15; 35Q15; 15B52; 33E17

\section{Introduction and motivation}

We consider the Hankel determinant

$$
H_{n}(v, s, \alpha)=\operatorname{det}\left(\int_{\mathbb{R}} x^{j+k} w(x ; v, s, \alpha) \mathrm{d} x\right)_{j, k=0}^{n-1}, \quad n \in \mathbb{N},
$$

with a Gaussian weight on the real line of the form

$$
w(x ; v, s, \alpha)=e^{-x^{2}}|x-v|^{\alpha} \begin{cases}s, & \text { if } x<v, \\ 1, & \text { if } x>v,\end{cases}
$$

where $v \in \mathbb{R}, s \in[0,1]$ and $\alpha \in(-1, \infty)$. There is a root-type Fisher-Hartwig (FH) singularity if $\alpha \neq 0$. The piecewise constant factor in (1.2) is a jump-type FH singularity only if $s \neq 0$ and $s \neq 1$. If $s=1$ there is no jump, and if $s=0$ the weight is supported on the interval $[v, \infty)$. By Heine's formula, $H_{n}(v, s, \alpha)$ admits the following $n$-fold integral representation:

$$
H_{n}(v, s, \alpha)=\frac{1}{n !} \int_{\mathbb{R}^{n}} \Delta(x)^{2} \prod_{i=1}^{n} w\left(x_{i} ; v, s, \alpha\right) \mathrm{d} x_{i}, \quad \Delta(x)=\prod_{1 \leq i<j \leq n}\left(x_{j}-x_{i}\right) .
$$

In this paper we are interested in large $n$ asymptotics for $H_{n}(v, s, \alpha)$, uniformly in $s \in[0,1]$. An analogous case was analysed in [6] for Fredholm determinant associated with the sine kernel

This paper is a contribution to the Special Issue on Orthogonal Polynomials, Special Functions and Applications (OPSFA14). The full collection is available at https://www.emis.de/journals/SIGMA/OPSFA2017.html 
and in $[10,11]$ for Toeplitz determinants with a weight defined on the unit circle. We briefly summarize here some known results for particular values of the parameters and we present some applications.

The Hankel determinant $H_{n}(v, s, \alpha)$ arises in random matrix theory. Consider the set of $n \times n$ Hermitian matrices $M$ endowed with the probability measure

$$
\frac{1}{\widehat{Z}_{n}(v, \alpha)}|\operatorname{det}(M)-v I|^{\alpha} e^{-\operatorname{Tr} M^{2}} \mathrm{~d} M, \quad \mathrm{~d} M=\prod_{i=1}^{n} \mathrm{~d} M_{i i} \prod_{1 \leq i<j \leq n} \mathrm{~d} \Re M_{i j} \mathrm{~d} \Im M_{i j},
$$

where $\widehat{Z}_{n}(v, \alpha)$ is the normalisation constant. We will refer to this random matrix ensemble as the generalised Gaussian unitary ensemble, which we denote by $\operatorname{GUE}(v, \alpha)$. Such a measure of matrices $M$ induces a probability measure on the eigenvalues $x_{1}, \ldots, x_{n}$ of $M$ which is of the form

$$
\frac{1}{n ! Z_{n}(v, \alpha)} \Delta(x)^{2} \prod_{i=1}^{n} w\left(x_{i} ; v, 1, \alpha\right) \mathrm{d} x_{i},
$$

where $Z_{n}(v, \alpha)$ is called the partition function of $\operatorname{GUE}(v, \alpha)$. From (1.4) and the integral representation for Hankel determinants given by (1.3), we have the relation

$$
Z_{n}(v, \alpha)=H_{n}(v, 1, \alpha) \text {. }
$$

The special case of $\operatorname{GUE}(0,0)$ (note that if $\alpha=0$, the parameter $v$ is irrelevant) is called the Gaussian unitary ensemble (GUE), and has already been widely studied (see, e.g., [32]). The partition function of the GUE is a Selberg integral and is explicitly known (see, e.g., [32]). Its exact expression and its large $n$ asymptotics are given by

$$
\begin{aligned}
\log H_{n}(0,1,0) & =-\frac{n^{2}}{2} \log 2+\frac{n}{2} \log (2 \pi)+\sum_{j=1}^{n-1} \log (j !) \\
& =\frac{n^{2}}{2} \log \left(\frac{n}{2}\right)-\frac{3}{4} n^{2}+n \log (2 \pi)-\frac{\log n}{12}+\zeta^{\prime}(-1)+\mathcal{O}\left(n^{-1}\right), \quad \text { as } n \rightarrow \infty
\end{aligned}
$$

where $\zeta$ is Riemann's zeta-function.

When $\alpha \neq 0$ but $v=0$, the partition function of $\operatorname{GUE}(0, \alpha)$ is again a Selberg integral and is also known exactly for finite $n$, see [33] or [15, equation (A.8)]. This is not true for $v \neq 0$. In [29], Krasovsky obtained via the Riemann-Hilbert method that large $n$ asymptotics of $H_{n}(\sqrt{2 n} t, 1, \alpha)$, when $t$ is in a compact subset of $(-1,1)$, are given by

$$
\begin{aligned}
\log \frac{H_{n}(\sqrt{2 n} t, 1, \alpha)}{H_{n}(0,1,0)}= & \frac{\alpha}{2} n \log n-\frac{\alpha}{2}\left(1-2 t^{2}+\log 2\right) n+\frac{\alpha^{2}}{4} \log n+\frac{\alpha^{2}}{4} \log \left(2 \sqrt{1-t^{2}}\right) \\
& +\log \frac{G\left(1+\frac{\alpha}{2}\right)^{2}}{G(1+\alpha)}+\mathcal{O}\left(\frac{\log n}{n}\right)
\end{aligned}
$$

where $G$ is Barnes' $G$-function.

Let us denote by $x_{\min }^{(v, \alpha)}$ and $x_{\max }^{(v, \alpha)}$ the smallest and largest eigenvalue in $\operatorname{GUE}(v, \alpha)$, respectively. The probability of observing no eigenvalues in $(-\infty, v)$, denoted by $\mathbb{P}\left(x_{\min }^{(v, \alpha)} \geq v\right)$, can be expressed as a ratio of Hankel determinants given by (1.1) with $s=0$ and $s=1$. From a direct integration of (1.4), and from the symmetry $w(x ; v, 1, \alpha)=w(-x ;-v, 1, \alpha)$, we have

$$
\mathbb{P}\left(x_{\min }^{(v, \alpha)} \geq v\right)=\frac{H_{n}(v, 0, \alpha)}{H_{n}(v, 1, \alpha)}=\mathbb{P}\left(x_{\max }^{(-v, \alpha)} \leq-v\right) .
$$


It is well-known that the empirical spectral distribution of the eigenvalues (after proper rescaling) in the GUE converges weakly almost surely to the Wigner semi-circle distribution, i.e.,

$$
\frac{1}{n} \sum_{i=1}^{n} \delta_{\frac{x_{i}}{\sqrt{2 n}}} \rightarrow \frac{2}{\pi} \sqrt{1-x^{2}} \mathrm{~d} x,
$$

see for instance [1, Chapter 2]. It is also known that the smallest eigenvalue $x_{\min }^{(0,0)}$ is usually located near $-\sqrt{2 n}$ and the properly rescaled fluctuations of $x_{\min }^{(0,0)}$ around $-\sqrt{2 n}$ follow the Tracy-Widom distribution. The ratio (1.7) with $v=-\sqrt{2 n}\left(1+\frac{w}{2 n^{2 / 3}}\right)$ and $w$ in a compact subset of $\mathbb{R}$, i.e., when $v$ is near the edge of the spectrum, has been recently studied for $\alpha \neq 0$ in [41] (including general $s>0$ ).

In this paper we investigate the probability of a large deviation of $x_{\min }^{(v, \alpha)}$, i.e., the probability (1.7) when $v$ is sufficiently far from $-\sqrt{2 n}$. In the particular case of $v=0,(1.7)$ is the probability that a matrix $M$ drawn from the $\operatorname{GUE}(0, \alpha)$ is positive definite. Large $n$ asymptotics for this probability have been obtained in [15]:

$$
\log \frac{H_{n}(0,0, \alpha)}{H_{n}(0,1, \alpha)}=-\frac{\log 3}{2} n^{2}-\frac{\alpha \log 3}{2} n+\left(\frac{\alpha^{2}}{4}-\frac{1}{12}\right) \log n+c_{0}+\mathcal{O}\left(n^{-1}\right),
$$

where

$$
c_{0}=\frac{\alpha}{2} \log (2 \pi)+\left(\frac{\alpha^{2}}{4}-\frac{1}{6}\right) \log 2+\left(\frac{1}{8}-\frac{\alpha^{2}}{2}\right) \log 3+\zeta^{\prime}(-1)-\log \left[G\left(1+\frac{\alpha}{2}\right)^{2}\right] .
$$

Note that the denominator $H_{n}(0,1, \alpha)$ can be obtained from (1.5) and (1.6) with $t=0$. One of the goals of the present paper is to generalize this result for $v \neq 0$, i.e., to obtain strong large $n$ asymptotics of $H_{n}(\sqrt{2 n} t, 0, \alpha)$, when $t$ is in a compact subset of $(-1, \infty)$. In particular, it is possible to deduce from our result the probability (1.7).

Assume we thin the eigenvalues $x_{1}, \ldots, x_{n}$ from (1.4) by removing each of them independently with a certain probability $s \in[0,1]$. The resulting point process is called the thinned $\operatorname{GUE}(v, \alpha)$, whose spectrum is denoted by $y_{1}, \ldots, y_{m}$, where $m$ is itself a random variable following the Binomial distribution $\operatorname{Bin}(n, 1-s)$. Thinning was introduced in random matrix theory by Bohigas and Pato [5], and we refer to [9, 11] for analogous situations and an overview of the theory of thinning. Let us denote by $y_{\mathrm{min}}^{(v, s, \alpha)}$ for the smallest thinned eigenvalue (note that we always have $\left.y_{\min }^{(v, s, \alpha)} \geq x_{\min }^{(v, \alpha)}=y_{\min }^{(v, 0, \alpha)}\right)$. The ratio $\frac{H_{n}(v, s, \alpha)}{H_{n}(v, 1, \alpha)}$ is a one-parameter generalisation of (1.7) and represents the probability of observing no eigenvalue of the thinned spectrum in $(-\infty, v)$, i.e., we have

$$
\mathbb{P}\left(y_{\min }^{(v, s, \alpha)} \geq v\right)=\frac{H_{n}(v, s, \alpha)}{H_{n}(v, 1, \alpha)} .
$$

In the regime when $s$ is in a compact subset of $(0,1]$ and $v=\sqrt{2 n} t$ with $t$ in a compact subset of $(-1,1)$, asymptotics of this probability have been obtained rigorously in [27] for $\alpha=0$ and in [9] for $\alpha \neq 0$. Note that asymptotics for integer $\alpha$ were obtained previously in [25], based on the work [7]. As $n \rightarrow \infty$, they are given by

$$
\begin{gathered}
\log \frac{H_{n}(\sqrt{2 n} t, s, \alpha)}{H_{n}(\sqrt{2 n} t, 1, \alpha)}=n \int_{-1}^{t} \frac{2}{\pi} \sqrt{1-x^{2}} \mathrm{~d} x \log s+\frac{(\log s)^{2}}{4 \pi^{2}} \log n+\tilde{c}_{0}+\mathcal{O}\left(\frac{\log n}{n}\right) \\
=\left[2 \arcsin t+2 t \sqrt{1-t^{2}}+\pi\right] \frac{\log s}{2 \pi} n+\frac{(\log s)^{2}}{4 \pi^{2}} \log n+\tilde{c}_{0}+\mathcal{O}\left(\frac{\log n}{n}\right)
\end{gathered}
$$


where the constant $\tilde{c}_{0}=\tilde{c}_{0}(t, s, \alpha)$ is explicit:

$$
\tilde{c}_{0}=\frac{3(\log s)^{2}}{4 \pi^{2}} \log \left(2 \sqrt{1-t^{2}}\right)+\alpha \frac{\log s}{2 \pi} \arcsin t+\log \frac{G\left(1+\frac{\alpha}{2}+\frac{\log s}{2 \pi i}\right) G\left(1+\frac{\alpha}{2}-\frac{\log s}{2 \pi i}\right)}{G\left(1+\frac{\alpha}{2}\right)^{2}} .
$$

The second goal of the present paper is to obtain large $n$ asymptotics of $H_{n}(\sqrt{2 n} t, s, \alpha)$ when $s=s(n) \rightarrow 0$ as $n \rightarrow \infty$, and to observe a transition in the large $n$ asymptotics between (1.10), where $s$ is bounded away from 0 , and the case $H_{n}(\sqrt{2 n} t, 0, \alpha)$.

Another motivation for the study of the Hankel determinant (1.1) comes from solutions of the Painlevé IV differential equation $\left(\mathrm{P}_{\mathrm{IV}}\right)$. The $\mathrm{P}_{\mathrm{IV}}$ equation finds interesting applications in many different areas of physics, such as non-linear optics, dispersive long-wave equations, fluid dynamics and plasma physics (see, e.g., [13, Section 10.1], [40]). This equation depends on two parameters $A, B \in \mathbb{C}$ and is given by

$$
q^{\prime \prime}(z)=\frac{1}{2 q(z)} q^{\prime}(z)^{2}+\frac{3}{2} q(z)^{3}+4 z q(z)^{2}+2\left(z^{2}-A\right) q(z)+\frac{B}{q(z)} .
$$

In this paper, we focus on special function solutions of $\mathrm{P}_{\mathrm{IV}}$ when the parameters $A$ and $B$ satisfy suitable constraints. It is known (see, e.g., [14, Theorem 3.4] and [26, Theorem 25.2]) that $\mathrm{P}_{\mathrm{IV}}$ has solutions expressible in terms of parabolic cylinder functions $U(a, z)$ (these functions are defined in [36, Chapter 12]) if and only if either

$$
B=-2(2 n+1+\varepsilon A)^{2} \quad \text { or } \quad B=-2 n^{2},
$$

with $\varepsilon= \pm 1$ and $n \in \mathbb{Z}$. In this situation, we are considering the so-called special function solutions of $\mathrm{P}_{\mathrm{IV}}$.

In general, the standard method to derive special function solutions is by considering associated Riccati equations. We refer the reader to [13, Section 7] for the general theory or [36, Section 32.10] for a summary of special function solutions. For $n=0$ in (1.11), the Riccati equation of $\mathrm{P}_{\mathrm{IV}}$ is

$$
q^{\prime}(z)=\varepsilon q(z)^{2}+2 \varepsilon z q(z)+2 \nu,
$$

with $\nu=-(1+\varepsilon A)$. If we set $q(z)=-\varepsilon \varphi_{\nu}^{\prime}(z) / \varphi_{\nu}(z)$ to linearise this equation, then $\varphi_{\nu}$ satisfies

$$
\varphi_{\nu}^{\prime \prime}(z)-2 \varepsilon z \varphi_{\nu}^{\prime}(z)+2 \varepsilon \nu \varphi_{\nu}(z)=0
$$

whose general solutions can be written in terms of the parabolic cylinder function $U(a, z)$, see $\left[36\right.$, Section 12.2]. If $\nu \notin \mathbb{Z}$, then $\varphi_{\nu}(z)=\varphi_{\nu}(z ; \varepsilon)$ can be written in the form

$$
\varphi_{\nu}(z ; \varepsilon)= \begin{cases}\left\{C_{1} U\left(-\nu-\frac{1}{2}, \sqrt{2} z\right)+C_{2} U\left(-\nu-\frac{1}{2},-\sqrt{2} z\right)\right\} \exp \left(\frac{1}{2} z^{2}\right), & \text { if } \varepsilon=1, \\ \left\{C_{1} U\left(\nu+\frac{1}{2}, \sqrt{2} z\right)+C_{2} U\left(\nu+\frac{1}{2},-\sqrt{2} z\right)\right\} \exp \left(-\frac{1}{2} z^{2}\right), & \text { if } \varepsilon=-1,\end{cases}
$$

where $C_{1}, C_{2} \in \mathbb{C}$. We comment on the case $\nu \in \mathbb{Z}$ at the end of this section. For general $n \in \mathbb{N}$, it is a remarkable fact that the special function solutions can be constructed explicitly in terms of the following Wronskian determinants (see [23, 35] and [14, Theorem 3.5]):

$$
\tau_{n, \nu}(z ; \varepsilon)=\mathcal{W}\left(\varphi_{\nu}(z ; \varepsilon), \frac{\mathrm{d} \varphi_{\nu}(z ; \varepsilon)}{\mathrm{d} z}, \ldots, \frac{\mathrm{d}^{n-1} \varphi_{\nu}(z ; \varepsilon)}{\mathrm{d} z^{n-1}}\right), \quad n \geq 1
$$

with $\tau_{0, \nu}(z ; \varepsilon)=1$. This Wronskian determinant can in turn be written in terms of the Hankel determinant $H_{n}(v, s, \alpha)$, we obtain for $n \geq 0$

$$
H_{n}(v, s, \alpha)=\Gamma(1+\alpha)^{n} 2^{-n^{2}-\frac{n(\alpha-1)}{2}} \tau_{n, \alpha}(v,-1)
$$




$$
=e^{-n v^{2}} \Gamma(1+\alpha)^{n} 2^{-n^{2}-\frac{n(\alpha-1)}{2}} \tau_{n,-\alpha-1}(v, 1),
$$

where $C_{1}=1$ and $C_{2}=s$. This relation relies on the integral representation for the parabolic cylinder function [36, equation (12.5.1)]:

$$
U(a, z)=\frac{e^{-\frac{z^{2}}{4}}}{\Gamma\left(a+\frac{1}{2}\right)} \int_{0}^{\infty} x^{a-\frac{1}{2}} e^{-\frac{x^{2}}{2}-z x} \mathrm{~d} x, \quad \Re a>-\frac{1}{2} .
$$

By a change of variables, we can write the moment of order 0 of the weight function $w(x)=$ $w(x ; v, s, \alpha)$ given by (1.2) in terms of $U$ as follows:

$$
\begin{aligned}
\int_{-\infty}^{\infty} w(x) \mathrm{d} x & =2^{-\frac{1+\alpha}{2}} e^{-v^{2}}\left[s \int_{0}^{\infty} x^{\alpha} e^{-\frac{x^{2}}{2}+\sqrt{2} v x} \mathrm{~d} x+\int_{0}^{\infty} x^{\alpha} e^{-\frac{x^{2}}{2}-\sqrt{2} v x} \mathrm{~d} x\right] \\
& =2^{-\frac{1+\alpha}{2}} e^{-\frac{v^{2}}{2}} \Gamma(1+\alpha)\left[s U\left(\alpha+\frac{1}{2},-\sqrt{2} v\right)+U\left(\alpha+\frac{1}{2}, \sqrt{2} v\right)\right] .
\end{aligned}
$$

Therefore, from (1.13) with $C_{1}=1$ and $C_{2}=s$, we have

$$
\int_{-\infty}^{\infty} w(x) \mathrm{d} x=2^{-\frac{1+\alpha}{2}} \Gamma(1+\alpha) \varphi_{\alpha}(v ;-1)=2^{-\frac{1+\alpha}{2}} e^{-v^{2}} \Gamma(\alpha+1) \varphi_{-\alpha-1}(v ; 1) .
$$

Differentiating $j$ times (1.16) with respect to $v$, we get

$$
\begin{aligned}
& \partial_{v}^{j} \varphi_{\alpha}(v ;-1)=\frac{2^{\frac{1+\alpha}{2}}}{\Gamma(1+\alpha)} \partial_{v}^{j}\left(\int_{-\infty}^{\infty} w(x) \mathrm{d} x\right)=\frac{(-1)^{j} 2^{j} 2^{\frac{1+\alpha}{2}}}{\Gamma(1+\alpha)} \int_{-\infty}^{\infty} x^{j} w(x) \mathrm{d} x, \\
& \partial_{v}^{j} \varphi_{-\alpha-1}(v ; 1)=\frac{2^{\frac{1+\alpha}{2}}}{\Gamma(1+\alpha)} \partial_{v}^{j}\left(e^{v^{2}} \int_{-\infty}^{\infty} w(x) \mathrm{d} x\right)=\frac{(-1)^{j} 2^{j} 2^{\frac{1+\alpha}{2}}}{\Gamma(1+\alpha)} e^{v^{2}} \int_{-\infty}^{\infty}(x-v)^{j} w(x) \mathrm{d} x .
\end{aligned}
$$

After taking the determinant, this establishes the formula (1.14). Thus, the results presented in Section 2 imply large $n$ asymptotics for special solutions of $\mathrm{P}_{\mathrm{IV}}$ expressed in terms of $\tau_{n, \nu}$, uniformly for $C_{2}$ small.

As explained in [14], when the parameter $\nu=m$ is an integer, we need to take a different combination of independent solutions of equation (1.12), since both parabolic cylinder functions in the seed function become Hermite polynomials. This leads to the so-called generalised Hermite polynomials, which are rational solutions of $\mathrm{P}_{\mathrm{IV}}$, obtained as particular cases of the special function solutions, see the parameter plane of $\mathrm{P}_{\mathrm{IV}}$ in [13, Section 5.5] or [26]. We refer the reader to the work of Kawijara and Ohta [28, Definition 3.1] for more information on the rational solutions of $\mathrm{P}_{\mathrm{IV}}$.

We also note that in the previous discussion, since $\nu=\alpha$, the integral representation (1.15) imposes the restriction $\alpha>-1$. This restriction can be lifted at the price of taking complex integration and a complex weight function. This defines an associated family of orthogonal polynomials only formally. We do not pursue this route in this paper, but we note that it has been used in the literature, for example in the analysis of the asymptotic behavior and pole structure of rational solutions of $\mathrm{P}_{\mathrm{II}}$ by Bertola and Bothner [3] and more recently of rational solutions of $\mathrm{P}_{\mathrm{IV}}$ by Buckingham [8].

\section{Main results}

Observe that if we naively take the limit $s \rightarrow 0$ in (1.10), the asymptotics on the right-hand side blow up, due to the presence of $\log s$ terms. Therefore, a critical transition is expected when $n \rightarrow \infty$ and simultaneously $s \rightarrow 0$ in a suitable double scaling limit. As mentioned earlier, the contribution of this paper is to analyse 1) the case $s=0$ (which is known only for $v=0$, see (1.8)), and 2) the transition between the situation when $s=0$ and the situation when $s$ is in a compact subset of $(0,1]$, given by $(1.10)$. We obtain the following results. 
Theorem 2.1. Let $\alpha \in(-1, \infty)$ and $t \in(-1, \infty)$. As $n \rightarrow \infty$, we have

$$
\log \frac{H_{n}(\sqrt{2 n} t, 0, \alpha)}{H_{n}(0,0, \alpha)}=C_{1}(t) n^{2}+C_{2}(t, \alpha) n+C_{3}(t, \alpha)+\mathcal{O}\left(n^{-1}\right),
$$

where the coefficients are given by

$$
\begin{aligned}
C_{1}(t)=- & \frac{2 t^{3}}{27}\left(\sqrt{3+t^{2}}-t\right)-\left(\frac{4}{3} t^{2}+\frac{5}{9} t \sqrt{3+t^{2}}\right)-\log \left(\frac{t+\sqrt{3+t^{2}}}{\sqrt{3}}\right), \\
C_{2}(t, \alpha)= & \frac{\alpha t}{3}\left(t-\sqrt{3+t^{2}}\right)-\alpha \log \left(\frac{t+\sqrt{3+t^{2}}}{\sqrt{3}}\right), \\
C_{3}(t, \alpha)= & \frac{1-3 \alpha^{2}}{6} \log \left(\frac{t+\sqrt{3+t^{2}}}{\sqrt{3}}\right)-\frac{1}{48} \log \left(\frac{3+t^{2}}{3}\right) \\
& -\frac{1}{16}\left(1-4 \alpha^{2}\right) \log \left(\frac{3+5 t^{2}+4 t \sqrt{3+t^{2}}}{3}\right) .
\end{aligned}
$$

Furthermore, the error term $\mathcal{O}\left(n^{-1}\right)$ is uniform for $t$ in a compact subset of $(-1, \infty)$.

Note that there is no critical transition in (2.1) as $t \rightarrow 1$.

Remark 2.2. The probability (1.7) with $v=\sqrt{2 n} t$ can be rewritten as

$$
\mathbb{P}\left(x_{\min }^{(\sqrt{2 n} t, \alpha)} \geq \sqrt{2 n} t\right)=\frac{H_{n}(\sqrt{2 n} t, 0, \alpha)}{H_{n}(0,0, \alpha)} \frac{H_{n}(0,0, \alpha)}{H_{n}(0,1, \alpha)} \frac{H_{n}(0,1, \alpha)}{H_{n}(\sqrt{2 n} t, 1, \alpha)} .
$$

Large $n$ asymptotics of these three ratios are given up to the constant term by Theorem 2.1 (for $t>-1$ ), (1.8) and (1.6) (for $t \in(-1,1)$ ), respectively. Putting these asymptotics together, we obtain as $n \rightarrow \infty$ and for $t \in(-1,1)$,

$$
\begin{aligned}
& \log \mathbb{P}\left(x_{\min }^{(\sqrt{2 n} t, \alpha)} \geq \sqrt{2 n} t\right)=\left(C_{1}(t)-\frac{\log 3}{2}\right) n^{2}+\left(C_{2}(t, \alpha)-\frac{\alpha \log 3}{2}-\alpha t^{2}\right) n \\
&+\left(\frac{\alpha^{2}}{4}-\frac{1}{12}\right) \log n+C_{3}(t, \alpha)+c_{0}-\frac{\alpha^{2}}{8} \log \left(1-t^{2}\right)+\mathcal{O}\left(\frac{\log n}{n}\right) .
\end{aligned}
$$

It can be checked from $(2.2)$ that $C_{1}(-1)=\frac{\log 3}{2}$ and

$$
C_{1}^{\prime}(t)=-\frac{8}{27}\left(\sqrt{3+t^{2}}-t\right)\left(3+5 t^{2}+4 t \sqrt{3+t^{2}}\right)<0, \quad \text { for } \quad t>-1,
$$

which shows that the leading term in (2.5) is negative. This implies that the above probability decays super exponentially fast as $n \rightarrow \infty$ for $t \in(-1,1)$.

To observe a transition in the large $n$ asymptotics of $H_{n}(\sqrt{2 n} t, s, \alpha)$ when $s=0$ and when $s$ is in a compact subset of $(0,1]$, we couple the parameter $s$ with $n$ in the form

$$
s=e^{-\lambda n}, \quad \lambda \geq 0 .
$$

Large $n$ asymptotics of $H_{n}\left(\sqrt{2 n} t, e^{-\lambda n}, \alpha\right)$ will depend on whether $\lambda$ is greater or smaller than a critical value $\lambda_{c}(t)$, which is explicit and given by

$$
\begin{aligned}
\lambda_{c}(t)= & \frac{2 t}{\sqrt{3}} \sqrt{3+t^{2}+2 t \sqrt{3+t^{2}}} \\
& +2 \log \left(2+t^{2}+t \sqrt{3+t^{2}}+\frac{\sqrt{3+t^{2}}+t}{\sqrt{3}} \sqrt{3+t^{2}+2 t \sqrt{3+t^{2}}}\right) .
\end{aligned}
$$


Theorem 2.3. Let $s=e^{-\lambda n}$ with $\lambda \in[0, \infty)$, we have the following asymptotic results.

(1) If $t \in(-1,1)$ and $\lambda \geq \lambda_{c}(t)$, then

$$
\log \frac{H_{n}\left(\sqrt{2 n} t, e^{-\lambda n}, \alpha\right)}{H_{n}(\sqrt{2 n} t, 0, \alpha)}=\mathcal{O}\left(n^{-1 / 2} e^{-n\left(\lambda-\lambda_{c}(t)\right)}\right), \quad \text { as } \quad n \rightarrow \infty,
$$

and large $n$ asymptotics for $\log H_{n}(\sqrt{2 n} t, 0, \alpha)$ are given by Theorem 2.1. Furthermore, the $\mathcal{O}$ term in $(2.7)$ is uniform for $t$ in a compact subset of $(-1,1)$ and for $\lambda \geq \lambda_{c}(t)$.

(2) If $t \in(-1,1)$ and $0 \leq \lambda \leq \lambda_{c}(t)$ are fixed, then

$$
\lim _{n \rightarrow \infty} \frac{1}{n^{2}} \log \frac{H_{n}\left(\sqrt{2 n} t, e^{-\lambda n}, \alpha\right)}{H_{n}(\sqrt{2 n} t, 1, \alpha)}=-\int_{0}^{\lambda} \Omega(t, \tilde{\lambda}) \mathrm{d} \tilde{\lambda}
$$

where

$$
\Omega(t, \lambda)=\int_{a}^{b} \rho(x ; t, \lambda) \mathrm{d} x, \quad \rho(x ; t, \lambda)=\frac{2}{\pi} \sqrt{c-x} \sqrt{\frac{x-b}{x-t}} \sqrt{x-a},
$$

and $a<b<t<c$, with $a, b$ and $c$ depending on $\lambda$ and $t$, are uniquely determined by the following equations:

$$
\begin{aligned}
& t=a+b+c, \\
& 2=a^{2}+b^{2}+c^{2}-t^{2}, \\
& \lambda=4 \int_{b}^{t} \frac{\sqrt{c-x}}{\sqrt{t-x}} \sqrt{x-b} \sqrt{x-a} \mathrm{~d} x .
\end{aligned}
$$

Remark 2.4. In Theorem 2.3, we restrict ourselves to the case $t \in(-1,1)$. With increasing effort, this result can be extended for $t \in(-1, \infty)$. If $t \geq 1$, a new region appears in the $(t, \lambda)$ plane which deserves a separate analysis (which we expect to be straightforward but long). Therefore, we decided not to proceed in this direction.

Remark 2.5. Note that the denominators on the left hand sides of (2.7) and (2.8) are different. We can use Theorem 2.3 to obtain information about the large deviation of the smallest thinned $\operatorname{GUE}(\sqrt{2 n} t, \alpha)$ eigenvalue as follows. By (1.9) and (1.7) with $v=\sqrt{2 n} t$ and $s=e^{-\lambda n}$, we have

$$
\mathbb{P}\left(y_{\min }^{\left(\sqrt{2 n} t, e^{-\lambda n}, \alpha\right)} \geq \sqrt{2 n} t\right)=\frac{H_{n}\left(\sqrt{2 n} t, e^{-\lambda n}, \alpha\right)}{H_{n}(\sqrt{2 n} t, 0, \alpha)} \mathbb{P}\left(x_{\min }^{(\sqrt{2 n} t, \alpha)} \geq \sqrt{2 n} t\right) .
$$

Therefore, for $t \in(-1,1)$ and $\lambda \geq \lambda_{c}(t)$, by $(2.7)$, as $n \rightarrow \infty$ we have

$$
\log \mathbb{P}\left(y_{\min }^{\left(\sqrt{2 n} t, e^{-\lambda n}, \alpha\right)} \geq \sqrt{2 n} t\right)=\log \mathbb{P}\left(x_{\min }^{(\sqrt{2 n} t, \alpha)} \geq \sqrt{2 n} t\right)+\mathcal{O}\left(n^{-1 / 2} e^{-n\left(\lambda-\lambda_{c}(t)\right)}\right),
$$

and large $n$ asymptotics of $\log \mathbb{P}\left(x_{\min }^{(\sqrt{2 n} t, \alpha)} \geq \sqrt{2 n} t\right)$ are given by $(2.5)$. In the regime $t \in(-1,1)$ and $0 \leq \lambda \leq \lambda_{c}(t),(2.8)$ implies

$$
\log \mathbb{P}\left(y_{\min }^{\left(\sqrt{2 n} t, e^{-\lambda n}, \alpha\right)} \geq \sqrt{2 n} t\right)=\left(-\int_{0}^{\lambda} \Omega(t, \tilde{\lambda}) \mathrm{d} \tilde{\lambda}\right) n^{2}+o\left(n^{2}\right) .
$$

Since (2.9) and (2.10) are both valid for $\lambda=\lambda_{c}(t)$, by equalling the leading term, we have

$$
-\int_{0}^{\lambda_{c}(t)} \Omega(t, \lambda) \mathrm{d} \lambda=C_{1}(t)-\frac{\log 3}{2} .
$$

We will give an independent and more direct proof of this formula at the end of Section 7 . 
Remark 2.6. Note that the limit (2.8) is independent of $\alpha$. The subleading terms in the large $n$ asymptotics of $\frac{H_{n}\left(\sqrt{2 n} t, e^{-\lambda n}, \alpha\right)}{H_{n}(\sqrt{2 n} t, 1, \alpha)}$ are expected to depend on $\alpha$ and to be oscillatory and described in terms of elliptic $\theta$-functions. These functions appear in our analysis (see, e.g., (6.12)). This heuristic is also supported by the analogy of our situation with [6], where the authors obtained $\theta$-functions in the subleading terms.

\section{Outline}

The orthogonal polynomials (OPs) with respect to the weight (1.2) play a central role in our analysis. In Section 3, we obtain identities for $\partial_{v} H_{n}(v, 0, \alpha)$ and for $\partial_{s} H_{n}(v, s, \alpha)$ in terms of these OPs. The Riemann-Hilbert $(\mathrm{RH})$ problem which characterizes these OPs is presented in Section 4. We obtain large $n$ asymptotics for the OPs via a Deift-Zhou steepest descent method on this RH problem. The first steps of the steepest descent method are the same regardless of the value of the parameter $s \in[0,1)$, and are also presented in Section 4 . For the last steps, the analysis will then depend on the speed of convergence of $s$ to 0 . By writing $s=e^{-\lambda n}, \lambda \in(0, \infty]$, we distinguish two different regimes in $\lambda$ which are separated by the critical value $\lambda_{c}(t)>0$. We study the situation $\lambda \geq \lambda_{c}(t)$ in Section 5 (the case $s=0$ corresponds to the special case of $\lambda=+\infty)$, and the situation $0<\lambda<\lambda_{c}(t)$ in Section 6. We integrate the differential identities and prove Theorem 2.1 and Theorem 2.3 in Section 7.

\section{Orthogonal polynomials and differential identities}

\subsection{Orthogonal polynomials}

We consider the family of orthonormal polynomials $p_{j}$ of degree $j$ with respect to $w$ defined in (1.2), characterized by the orthogonality conditions

$$
\int_{\mathbb{R}} p_{j}(x) p_{k}(x) w(x) \mathrm{d} x=\delta_{j k}, \quad j, k=0,1,2, \ldots,
$$

and $\kappa_{j}>0$ is the leading coefficient of $p_{j}$, that is $\pi_{j}(x)=\kappa_{j}^{-1} p_{j}(x)$ is the monic orthogonal polynomial of degree $j$ which satisfies

$$
\int_{\mathbb{R}} \pi_{j}(x) \pi_{k}(x) w(x) \mathrm{d} x=h_{j} \delta_{j k}, \quad j, k=0,1,2, \ldots,
$$

where $h_{j}$ is the squared norm of $\pi_{j}$. From (3.1), we have $h_{j}=\kappa_{j}^{-2}$. It is well-known (see, e.g., [38]) that these OPs satisfy the recurrence relation

$$
x \pi_{j}(x)=\pi_{j+1}(x)+\beta_{j} \pi_{j}(x)+\gamma_{j}^{2} \pi_{j-1}(x), \quad j \geq 0,
$$

with $\pi_{-1}(x):=0$. Note that if we write $\pi_{j}(x)=x^{j}+\sigma_{j} x^{j-1}+\cdots$, then from (3.3) we get the relation

$$
\sigma_{j}-\sigma_{j+1}=\beta_{j}, \quad j \geq 0, \quad \text { where } \quad \sigma_{0}:=0 .
$$

\subsection{Differential identity in $v$ for $s=0$}

From the determinantal representation for OPs (see, e.g., [38]) and (1.3), the Hankel determinant $H_{n}(v, 0, \alpha)$ can be written in terms of the norms of the OPs, one has

$$
H_{n}(v, 0, \alpha)=\prod_{j=0}^{n-1} h_{j} .
$$


If we differentiate with respect to $v$ the relation (3.2) with $k=j$, we obtain

$$
\partial_{v} h_{j}=\partial_{v}\left(\int_{v}^{\infty} \pi_{j}^{2}(x) w(x) \mathrm{d} x\right)=\partial_{v}\left(\int_{0}^{\infty} \pi_{j}^{2}(x+v) w(x+v) \mathrm{d} x\right) .
$$

Since $w(x+v)=x^{\alpha} e^{-(x+v)^{2}}$, we get

$$
\begin{aligned}
\partial_{v} h_{j} & =2 \int_{0}^{\infty} \pi_{j}(x+v) \partial_{v}\left(\pi_{j}(x+v)\right) w(x+v) \mathrm{d} x-2 \int_{0}^{\infty}(x+v) \pi_{j}^{2}(x+v) w(x+v) \mathrm{d} x \\
& =-2 \int_{v}^{\infty} x \pi_{j}^{2}(x) w(x) \mathrm{d} x
\end{aligned}
$$

where we have used the orthogonality (3.2) and the fact that $\partial_{v}\left(\pi_{j}(x+v)\right)$ is a polynomial of degree at most $j-1$. From the recurrence relation (3.3), we obtain

$$
\partial_{v} h_{j}=-2 \beta_{j} h_{j}
$$

As a consequence of this, by taking the $\log$ in (3.5) and differentiating it with respect to $v$, we have

$$
\partial_{v} \log H_{n}(v, 0, \alpha)=\sum_{j=0}^{n-1} \partial_{v} \log h_{j}=-2 \sum_{j=0}^{n-1} \beta_{j},
$$

This can be simplified by using (3.4), and gives

$$
\partial_{v} \log H_{n}(v, 0, \alpha)=2 \sigma_{n}(v),
$$

where $\sigma_{n}$ is the subleading coefficient of the polynomial $\pi_{n}(x)$, defined after (3.3), and we have explicitly written the dependence of $\sigma_{n}$ on $v$.

\subsection{Differential identity in $s$}

Suppose that the thinned eigenvalues $y_{1}, \ldots, y_{m}$ are observed and that $\sharp\left\{y_{i}: y_{i}<v\right\}=0$. From Bayes' formula, using (1.4) and (1.9), the distribution of the whole spectrum $x_{1}, \ldots, x_{n}$ conditionally on this event is given by

$$
\frac{1}{n ! H_{n}(v, s, \alpha)} \Delta(x)^{2} \prod_{i=1}^{n} w\left(x_{i} ; v, s, \alpha\right) \mathrm{d} x_{i} .
$$

Such point processes are called conditional, and were first considered in [11] on the unit circle and then on the real line in [9]. This point process is determinantal [16], and its correlation kernel is given by

$$
K_{n}(x, y)= \begin{cases}\sqrt{w(x) w(y)} \frac{\kappa_{n-1}}{\kappa_{n}} \frac{p_{n-1}(y) p_{n}(x)-p_{n-1}(x) p_{n}(y)}{x-y}, & \text { if } x \neq y \\ w(x) \frac{\kappa_{n-1}}{\kappa_{n}}\left(p_{n}^{\prime}(x) p_{n-1}(x)-p_{n}(x) p_{n-1}^{\prime}(x)\right), & \text { if } x=y\end{cases}
$$

where the OPs $p_{j}$ are orthonormal with respect to $w(x ; v, s, \alpha)$ and are defined in (3.1). The expected number of points on $(-\infty, v)$ in this point process is denoted by $\mathcal{E}_{n}(v, s, \alpha)$. It is also known [16] that $\mathcal{E}_{n}(v, s, \alpha)$ can be expressed in terms of the one-point correlation function $K_{n}(x, x)$, we have

$$
\mathcal{E}_{n}(v, s, \alpha)=\int_{-\infty}^{v} K_{n}(x, x) \mathrm{d} x .
$$


The quantity $\mathcal{E}_{n}(v, s, \alpha)$ can also be expressed in terms of the logarithmic derivative of $H_{n}(v, s, \alpha)$ with respect to $s$. Consider the following partition of $\mathbb{R}^{n}$ :

$$
A_{k}=\left\{\left(x_{1}, \ldots, x_{n}\right) \in \mathbb{R}^{n}: \sharp\left\{x_{i}: x_{i}<v\right\}=k\right\}, \quad \bigsqcup_{k=0}^{n} A_{k}=\mathbb{R}^{n} .
$$

By definition of $\mathcal{E}_{n}(v, s, \alpha)$ we have

$$
\begin{aligned}
\mathcal{E}_{n}(v, s, \alpha) & =\sum_{k=0}^{n} \frac{k}{n ! H_{n}(v, s, \alpha)} \int_{A_{k}} \Delta(x)^{2} \prod_{i=1}^{n} w\left(x_{i} ; v, s, \alpha\right) \mathrm{d} x_{i} \\
& =\sum_{k=0}^{n} \frac{k s^{k}}{n ! H_{n}(v, s, \alpha)} \int_{A_{k}} \Delta(x)^{2} \prod_{i=1}^{n}\left|x_{i}-v\right|^{\alpha} e^{-x_{i}^{2}} \mathrm{~d} x_{i} .
\end{aligned}
$$

Note that the $n$-fold integral (1.3) can be rewritten as

$$
H_{n}(v, s, \alpha)=\sum_{k=0}^{n} \frac{s^{k}}{n !} \int_{A_{k}} \Delta(x)^{2} \prod_{i=1}^{n}\left|x_{i}-v\right|^{\alpha} e^{-x_{i}^{2}} \mathrm{~d} x_{i},
$$

and thus we have $\mathcal{E}_{n}(v, s, \alpha)=s \partial_{s} \log H_{n}(v, s, \alpha)$. Putting this together with (3.8), we obtain the differential identity

$$
s \partial_{s} \log H_{n}(v, s, \alpha)=\int_{-\infty}^{v} K_{n}(x, x) \mathrm{d} x .
$$

We will also use later the well-known (see, e.g., [16]) formula for reproducing kernels

$$
\int_{-\infty}^{\infty} K_{n}(x, x) \mathrm{d} x=n
$$

\section{A Riemann-Hilbert problem and renormalization of the problem}

We will perform the Deift-Zhou [20, 21] steepest descent method on a Riemann-Hilbert problem to get the large $n$ asymptotics for $p_{n}$. Consider the matrix valued function $Y$, defined by

$$
Y(z)=\left(\begin{array}{cr}
\kappa_{n}^{-1} p_{n}(z) & \frac{\kappa_{n}^{-1}}{2 \pi i} \int_{\mathbb{R}} \frac{p_{n}(x) w(x)}{x-z} \mathrm{~d} x \\
-2 \pi i \kappa_{n-1} p_{n-1}(z) & -\kappa_{n-1} \int_{\mathbb{R}} \frac{p_{n-1}(x) w(x)}{x-z} \mathrm{~d} x
\end{array}\right) .
$$

It is well-known [22] that $Y$ is the unique solution of the following $\mathrm{RH}$ problem.

\section{RH problem for $Y$}

(a) $Y: \mathbb{C} \backslash \mathbb{R} \rightarrow \mathbb{C}^{2 \times 2}$ is analytic.

(b) The limits of $Y(x \pm i \epsilon)$ as $\epsilon>0$ approaches 0 exist, are continuous on $\mathbb{R} \backslash\{v\}$ and are denoted by $Y_{+}$and $Y_{-}$respectively. Furthermore they are related by

$$
Y_{+}(x)=Y_{-}(x)\left(\begin{array}{cc}
1 & w(x) \\
0 & 1
\end{array}\right), \quad \text { for } \quad x \in \mathbb{R} \backslash\{v\} .
$$

(c) As $z \rightarrow \infty$, we have $Y(z)=\left(I+Y_{1} z^{-1}+\mathcal{O}\left(z^{-2}\right)\right) z^{n \sigma_{3}}$, where $\sigma_{3}=\left(\begin{array}{cc}1 & 0 \\ 0 & -1\end{array}\right)$. 
(d) As $z$ tends to $v$, the behaviour of $Y$ is

$$
\begin{aligned}
Y(z) & =\left(\begin{array}{ll}
\mathcal{O}(1) & \mathcal{O}(\log (z-v)) \\
\mathcal{O}(1) & \mathcal{O}(\log (z-v))
\end{array}\right), \quad \text { if } \quad \alpha=0 \\
Y(z) & =\left(\begin{array}{ll}
\mathcal{O}(1) & \mathcal{O}(1)+\mathcal{O}\left((z-v)^{\alpha}\right) \\
\mathcal{O}(1) & \mathcal{O}(1)+\mathcal{O}\left((z-v)^{\alpha}\right)
\end{array}\right), \quad \text { if } \alpha \neq 0 .
\end{aligned}
$$

If $s=0$, from condition (b) $Y$ has no jump along $(-\infty, v)$ and thus $Y$ is analytic in $\mathbb{C} \backslash[v, \infty)$. Note also that $Y_{11}(z)=\kappa_{n}^{-1} p_{n}(z)=\pi_{n}(z)$, and thus

$$
Y_{1,11}=\sigma_{n}(v)
$$

where $Y_{1,11}$ denotes the $(1,1)$ entry of the matrix $Y_{1}$.

\subsection{Normalization of the RH problem}

We define $t=\frac{v}{\sqrt{2 n}}$, and we normalize the RH problem for $Y$ with the following transformation

$$
U(z)=(2 n)^{-\left(\frac{\alpha}{4}+\frac{n}{2}\right) \sigma_{3}} Y(\sqrt{2 n} z)(2 n)^{\frac{\alpha}{4} \sigma_{3}} .
$$

The matrix $U$ satisfies the following $\mathrm{RH}$ problem.

\section{RH problem for $U$}

(a) $U: \mathbb{C} \backslash \mathbb{R} \rightarrow \mathbb{C}^{2 \times 2}$ is analytic.

(b) $U$ has the following jumps:

$$
U_{+}(x)=U_{-}(x)\left(\begin{array}{cc}
1 & \widetilde{w}(x) \\
0 & 1
\end{array}\right), \quad \text { for } \quad x \in \mathbb{R} \backslash\{t\}
$$

where

$$
\widetilde{w}(x)=(2 n)^{-\frac{\alpha}{2}} w(\sqrt{2 n} x)=|x-t|^{\alpha} e^{-2 n x^{2}} \begin{cases}s, & \text { if } x<t \\ 1, & \text { if } x>t\end{cases}
$$

(c) As $z \rightarrow \infty$, we have $U(z)=\left(I+U_{1} z^{-1}+\mathcal{O}\left(z^{-2}\right)\right) z^{n \sigma_{3}}$.

(d) As $z$ tends to $t$, the behaviour of $U$ is

$$
\begin{aligned}
U(z) & =\left(\begin{array}{ll}
\mathcal{O}(1) & \mathcal{O}(\log (z-t)) \\
\mathcal{O}(1) & \mathcal{O}(\log (z-t))
\end{array}\right), \quad \text { if } \quad \alpha=0 \\
U(z) & =\left(\begin{array}{ll}
\mathcal{O}(1) & \mathcal{O}(1)+\mathcal{O}\left((z-t)^{\alpha}\right) \\
\mathcal{O}(1) & \mathcal{O}(1)+\mathcal{O}\left((z-t)^{\alpha}\right)
\end{array}\right), \quad \text { if } \quad \alpha \neq 0 .
\end{aligned}
$$

The following lemma translates the differential identities (3.6) and (3.9) in terms of $U$.

Lemma 4.1. We have the following differential identities

$$
\begin{aligned}
& \partial_{t} \log H_{n}(\sqrt{2 n} t, 0, \alpha)=4 n U_{1,11} \\
& s \partial_{s} \log H_{n}(\sqrt{2 n} t, s, \alpha)=\int_{-\infty}^{t} \frac{\widetilde{w}(x)}{2 \pi i}\left[U^{-1}(x) U^{\prime}(x)\right]_{21} \mathrm{~d} x .
\end{aligned}
$$


Proof. The differential identity (4.5) is obtained by substituting (4.4) and (4.3) into (3.6). Similarly, using (3.7) and (4.1), the differential identity (3.9) can be rewritten as

$$
s \partial_{s} \log H_{n}(\sqrt{2 n} t, s, \alpha)=\int_{-\infty}^{\sqrt{2 n} t} \frac{w(x)}{2 \pi i}\left[Y^{-1}(x) Y^{\prime}(x)\right]_{21} \mathrm{~d} x,
$$

which gives (4.6) after using (4.4) and a change of variables.

Remark 4.2. Note that $\left[Y^{-1}(z) Y^{\prime}(z)\right]_{21}$ only involves the first column of $Y$, which is entire (see (4.1) or equivalently (4.2)). Thus $\left[Y_{+}^{-1}(x) Y_{+}^{\prime}(x)\right]_{21}=\left[Y_{-}^{-1}(x) Y_{-}^{\prime}(x)\right]_{21}$ for $x \in \mathbb{R}$, and we simply denote it by $\left[Y^{-1}(x) Y^{\prime}(x)\right]_{21}$ without ambiguity. The same remark holds for $U$.

\subsection{Equilibrium measure}

We introduce a new parameter $\lambda \in[0,+\infty]$, defined through $s=e^{-\lambda n}$, which characterizes the speed of convergence of $s$ to 0 as $n \rightarrow \infty$. An essential tool in the RH analysis is the so-called equilibrium measure. In our case, the equilibrium measure $\mu_{V}$ is the unique minimizer of the functional

$$
\iint_{\mathbb{R}^{2}} \log |x-y|^{-1} \mathrm{~d} \mu(x) \mathrm{d} \mu(y)+\int_{\mathbb{R}} V(x) \mathrm{d} \mu(x),
$$

among all Borel probability measures $\mu$ on $\mathbb{R}$, where the potential $V$ is defined by

$$
V(x)= \begin{cases}2 x^{2}+\lambda, & \text { if } x<t, \\ 2 x^{2}, & \text { if } x \geq t\end{cases}
$$

and where the parameter $t$ has been defined above (4.4). The equilibrium measure is absolutely continuous with respect to the Lebesgue measure and its density will be denoted by $\rho(x)$. The equilibrium measure and its support, denoted by $\mathcal{S}$, are completely determined by the following Euler-Lagrange variational conditions [37]:

$$
\begin{aligned}
& 2 \int_{\mathcal{S}} \log |x-y| \rho(y) \mathrm{d} y=V(x)-\ell, \quad \text { for } \quad x \in \mathcal{S}, \\
& 2 \int_{\mathcal{S}} \log |x-y| \rho(y) \mathrm{d} y \leq V(x)-\ell, \quad \text { for } \quad x \in \mathbb{R} \backslash \mathcal{S},
\end{aligned}
$$

where $\ell$ is a constant. Proposition 4.3 below shows that the equilibrium measure depends crucially on whether $\lambda \geq \lambda_{c}(t)$ or $0<\lambda<\lambda_{c}(t)$. If $\lambda=0$, the potential is simply $V(x)=2 x^{2}$ and the equilibrium measure is the semicircle law supported on $(-1,1)$ (see, e.g., [37]). For convenience we also include it in Proposition 4.3 (see case (3)), but without giving a proof of it.

\section{Proposition 4.3.}

(1) If $t \in(-1, \infty)$ and $\lambda \geq \lambda_{c}(t)$, the density of the equilibrium measure $\rho(x)=\rho(x ; t)$ is independent of $\lambda$ and is given by

$$
\rho(x)=\frac{2}{\pi}(x-\bar{b}) \frac{\sqrt{\bar{c}-x}}{\sqrt{x-t}},
$$

supported on $\mathcal{S}=[t, \bar{c}]$, with

$$
\bar{b}=\bar{b}(t)=\frac{t-\sqrt{3+t^{2}}}{3}, \quad \bar{c}=\bar{c}(t)=\frac{t+2 \sqrt{3+t^{2}}}{3} .
$$


The constant $\ell=\ell(t)$ in the variational conditions (4.7) and (4.8) is given by

$$
\ell=1+\frac{2}{3} t\left(\sqrt{3+t^{2}}+2 t\right)+2 \log \left(2\left(t+\sqrt{3+t^{2}}\right)\right) .
$$

Furthermore, the variational inequality (4.8) is strict for all $x \in \mathbb{R} \backslash \mathcal{S}$ if $\lambda>\lambda_{c}$, and if $\lambda=\lambda_{c}$ then (4.8) is strict for all $x \in \mathbb{R} \backslash(\mathcal{S} \cup\{\bar{b}\})$ and (4.8) is an equality at $x=\bar{b}$.

(2) If $t \in(-1,1)$ and $0<\lambda<\lambda_{c}(t)$, the density of the equilibrium measure $\rho(x)=\rho(x ; t, \lambda)$ is given by

$$
\rho(x)=\frac{2}{\pi} \sqrt{c-x} \sqrt{\frac{x-b}{x-t}} \sqrt{x-a},
$$

supported on two disjoint intervals

$$
\mathcal{S}=[a, b] \cup[t, c], \quad a<b<t<c,
$$

and $a, b, c$, depending on $\lambda$ and $t$, are uniquely determined by the following equations:

$$
\begin{aligned}
& t=a+b+c \\
& 2=a^{2}+b^{2}+c^{2}-t^{2}, \\
& \lambda=4 \int_{b}^{t} \frac{\sqrt{c-x}}{\sqrt{t-x}} \sqrt{x-b} \sqrt{x-a} \mathrm{~d} x .
\end{aligned}
$$

Furthermore, for a fixed $t$, the function $\lambda \mapsto b=b(a(\lambda), c(\lambda), \lambda)$ given by the system (4.12)-(4.14) is strictly decreasing from $\lambda \in\left(0, \lambda_{c}(t)\right)$ to $b \in(\bar{b}, t)$. The constant $\ell=\ell(t, \lambda)$ is given by

$$
\begin{aligned}
\ell & =-2 \int_{S} \log |x-t| \rho(x) \mathrm{d} x+2 t^{2} \\
& =-2 \log |c|+2 c^{2}+\int_{c}^{\infty}\left(4 x-\frac{2}{x}-4 \frac{\sqrt{x-c}}{\sqrt{x-t}} \sqrt{x-b} \sqrt{x-a}\right) \mathrm{d} x
\end{aligned}
$$

and (4.8) is strict.

(3) If $t \in(-1,1)$ and $\lambda=0$, then we have the semi-circle law

$$
\rho(x)=\frac{2}{\pi} \sqrt{1-x^{2}}, \quad \mathcal{S}=[-1,1], \quad \ell=1+\log 4,
$$

and (4.8) is strict for $x \in \mathbb{R} \backslash \mathcal{S}$.

Proof. We will start by proving case (2). From (4.12) and (4.13), we can express $a$ and $c$ in terms of $b$ and $t$ as follows

$$
a=-\frac{b}{2}+\frac{t}{2}-\frac{\sqrt{4-3 b^{2}+2 t b+t^{2}}}{2}, \quad c=-\frac{b}{2}+\frac{t}{2}+\frac{\sqrt{4-3 b^{2}+2 t b+t^{2}}}{2} .
$$

For $t \in(-1,1)$ fixed and $b \in(\bar{b}, t)$, a direct check from (4.17) shows that $a<b<t<c, \partial_{b} c<0$ and $-1<\partial_{b} a$. This implies from (4.14) that

$$
\partial_{b} \lambda=4 \int_{b}^{t} \frac{\sqrt{c-x}}{\sqrt{t-x}} \sqrt{x-b} \sqrt{x-a}\left(\frac{\partial_{b} c}{2(c-x)}-\frac{1}{2(x-b)}-\frac{\partial_{b} a}{2(x-a)}\right) \mathrm{d} x<0 .
$$


If $b \nearrow t$, equation (4.14) implies $\lambda \rightarrow 0$. On the other hand, if $b \searrow \bar{b}$, equations (4.12) and (4.13) imply $a \rightarrow \bar{b}$ and $c \rightarrow \bar{c}$. Again from (4.14), we thus have

$$
\lambda \rightarrow 4 \int_{\bar{b}}^{t} \frac{\sqrt{\bar{c}-x}}{\sqrt{t-x}}(x-\bar{b}) \mathrm{d} x=\lambda_{c}(t), \quad \text { as } \quad b \rightarrow \bar{b},
$$

where $\lambda_{c}(t)$ is given by (2.6). This proves that the function $\lambda \mapsto b(a(\lambda), c(\lambda), \lambda)$ is a decreasing bijection from $\lambda \in\left(0, \lambda_{c}(t)\right)$ to $b \in(\bar{b}, t)$. In particular, given $t \in(-1,1)$ and $0<\lambda<\lambda_{c}(t)$, $a, b$ and $c$ are uniquely determined by the equations (4.12)-(4.14). Equations (4.12) and (4.13) imply also that $\rho$ is a density. Indeed, with a contour deformation and a residue calculation at $\infty$, we obtain

$$
\int_{\mathcal{S}} \rho(x) \mathrm{d} x=\frac{a^{2}+b^{2}+c^{2}-2 a b-2 a c-2 b c+2(a+b+c) t-3 t^{2}}{4}=1 .
$$

Now, we define

$$
f(x)=2 \int_{\mathcal{S}} \log |x-y| \rho(y) \mathrm{d} y-2 x^{2},
$$

where $\rho$ is given by (4.11). Its derivative $f^{\prime}(x)$ can be explicitly evaluated. Consider the function $\tilde{\rho}(z)=\frac{2}{\pi} \sqrt{z-c} \frac{\sqrt{z-b}}{\sqrt{z-t}} \sqrt{z-a}$, such that $\tilde{\rho}_{ \pm}(x)= \pm i \rho(x)$ for $x \in \mathcal{S}$. From (4.2), we have that

$$
f^{\prime}(x)=\frac{1}{2 \pi i} \int_{\Sigma} \frac{-2 \pi \tilde{\rho}(w)}{x-w} \mathrm{~d} w-4 x
$$

where $\Sigma$ consists of two circles surrounding $\mathcal{S}$ in the counter-clockwise direction, and if $x \notin \mathcal{S}$, then $x$ does not lie in the interior region of $\Sigma$. Also, note that

$$
\operatorname{Res}\left(\frac{-2 \pi \tilde{\rho}(w)}{x-w}, w=\infty\right)=-2(a+b+c-t-2 x)=4 x,
$$

where we have used (4.12). Therefore, $f^{\prime}(x)=0$ for $x \in \mathcal{S}$ and from a contour deformation and a residue calculation, we obtain

$$
f^{\prime}(x)= \begin{cases}4 \frac{\sqrt{c-x}}{\sqrt{t-x}} \sqrt{b-x} \sqrt{a-x}, & x<a, \\ 0, & a \leq x \leq b, \\ -4 \frac{\sqrt{c-x}}{\sqrt{t-x}} \sqrt{x-b} \sqrt{x-a}, & b<x<t, \\ 0, & t \leq x \leq c, \\ -4 \frac{\sqrt{x-c}}{\sqrt{x-t}} \sqrt{x-b} \sqrt{x-a}, & c<x .\end{cases}
$$

Note furthermore that from (4.7), we have

$$
\lambda=-\int_{b}^{t} f^{\prime}(x) \mathrm{d} x=-(f(t)-f(b))
$$

This implies that (4.7) and (4.8) are satisfied, with a strict inequality in (4.8). From (4.7), we have $-\ell=f(x)$, for any $x \in[t, c]$. In particular we have

$$
\ell=-f(t)=-2 \int_{\mathcal{S}} \log |x-t| \rho(x) \mathrm{d} x+2 t^{2},
$$


which is (4.15). To prove the other expression for $\ell$ in (4.15), we note that

$$
f(x)=2 \log |x|-2 x^{2}+\tilde{f}(x),
$$

where $\tilde{f}(x)=2 \int_{\mathcal{S}} \log \left|1-\frac{y}{x}\right| \rho(y) \mathrm{d} y$. Using the fact that $\lim _{x \rightarrow \infty} \tilde{f}(x)=0$ and the equations (4.19) and (4.21), we obtain

$$
0=\tilde{f}(c)+\int_{c}^{\infty} \tilde{f}^{\prime}(x) \mathrm{d} x=-\ell-2 \log |c|+2 c^{2}+\int_{c}^{\infty}\left(4 x-\frac{2}{x}+f^{\prime}(x)\right) \mathrm{d} x,
$$

from which we find the second expression in (4.15). This finishes the proof of case (2).

The case (1) is similar and simpler. In this case, we define a function $f$ as in (4.2), where $\rho(x)=\frac{2}{\pi}(x-\bar{b}) \frac{\sqrt{c}-x}{\sqrt{x-t}}$. The derivative of $f$ is equal to

$$
f^{\prime}(x)= \begin{cases}-4 \frac{\sqrt{\bar{c}-x}}{\sqrt{t-x}}(x-\bar{b}), & x<t, \\ 0, & t \leq x \leq \bar{c}, \\ -4 \frac{\sqrt{x-\bar{c}}}{\sqrt{x-t}}(x-\bar{b}), & \bar{c}<x .\end{cases}
$$

The Euler-Lagrange constant can also be written as in (4.20), but in case (1) the integral can be explicitly evaluated with a primitive and gives (4.10). Since $\lambda \geq \lambda_{c}(t)$, from (4.22) and by the formula for $\lambda_{c}(t)$ given by (4.18), (4.8) is strictly satisfied in case (1) except at $x=\bar{b}$ if $\lambda=\lambda_{c}$. This finishes the proof of Proposition 4.3.

Remark 4.4. Here we just comment briefly on what happens for $t \geq 1$ in parts (2) and (3) of Proposition 4.3, even though we will only focus on $t \in(-1,1)$ in the present paper, as mentioned in Remark 2.4. If $t>1$, a critical situation occurs if the endpoints $t$ and $c$ merge together. From equations (4.12) and (4.13), in this case we have $a=-1$ and $b=1$. From (4.14), this corresponds to

$$
\lambda=4 \int_{1}^{t} \sqrt{x^{2}-1} \mathrm{~d} x=2 t \sqrt{t^{2}-1}-2 \log \left(t+\sqrt{t^{2}-1}\right)=: \epsilon(t) .
$$

With increasing effort (by adapting the above proof), it is possible to show that part (2) of Proposition 4.3 remains valid for $t \geq 1$, provided that $\lambda \in\left(\epsilon(t), \lambda_{c}(t)\right)$, and that part (3) remains valid for $t \geq 1$, provided that $\lambda \in[0, \epsilon(t)]$.

Remark 4.5. We will prove part 2 of Theorem 2.3 (that is, (2.8)) by using the differential identity (4.6) (after the change of variables $s=e^{-\lambda n}$ ). We a priori need large $n$ asymptotics of $\partial_{\lambda} \log H_{n}\left(\sqrt{2 n} t, e^{-\lambda n}, \alpha\right)$ uniformly in $\lambda \in\left[0, \lambda_{c}(t)\right]$. As it can be seen from Proposition 4.3, in this case the support of the equilibrium measure consists of two intervals. The region where $\lambda_{c}(t)-\lambda>0$ is small corresponds to the "birth of a cut", which was studied in [4, 12,34], and when $\lambda_{c}(t)-\lambda>0$ becomes larger, $\theta$-functions appear in the analysis. It is a technical task to obtain uniform asymptotics in these regions as $\lambda$ approaches $\lambda_{c}(t)$. Nevertheless, following [11], we will only need pointwise convergence in $\lambda \in\left(0, \lambda_{c}(t)\right)$ and apply Lebesgue's dominated convergence theorem.

\subsection{First transformation: $U \mapsto T$}

The first step of the steepest descent analysis consists of normalizing the $\mathrm{RH}$ problem at $\infty$, which can be done by using a so-called $g$-function. We define it by

$$
g(z)=\int_{\mathcal{S}} \log (z-y) \rho(y) \mathrm{d} y
$$


where the principal branch is chosen for the logarithm. The density $\rho$ and its support $\mathcal{S}$ are defined in Proposition 4.3. The $g$-function is analytic in $\mathbb{C} \backslash(-\infty, \sup \mathcal{S}]$ and possesses the following properties

$$
\begin{aligned}
& g_{+}(x)+g_{-}(x)=2 \int_{S} \log |x-y| \rho(y) \mathrm{d} y, \quad x \in \mathbb{R}, \\
& g_{+}(x)-g_{-}(x)=2 \pi i, \quad x<\inf \mathcal{S}, \\
& g_{+}(x)-g_{-}(x)=2 \pi i \int_{x}^{\sup \mathcal{S}} \rho(y) \mathrm{d} y, \quad x \in[\inf \mathcal{S}, \sup \mathcal{S}], \\
& g_{+}(x)-g_{-}(x)=0, \quad \sup \mathcal{S}<x,
\end{aligned}
$$

Furthermore, by expanding the $g$-function as $z \rightarrow \infty$ in (4.23), we have

$$
e^{n g(z)}=z^{n}\left(1-\frac{n}{z} \int_{\mathcal{S}} x \rho(x) \mathrm{d} x+\mathcal{O}\left(z^{-2}\right)\right), \quad z \rightarrow \infty .
$$

We apply a first transformation on $U$ by

$$
T(z)=e^{\frac{n \ell}{2} \sigma_{3}} U(z) e^{-n g(z) \sigma_{3}} e^{-\frac{n \ell}{2} \sigma_{3}} .
$$

$T$ satisfies the following RH problem.

\section{RH problem for $T$}

(a) $T: \mathbb{C} \backslash \mathbb{R} \rightarrow \mathbb{C}^{2 \times 2}$ is analytic.

(b) $T$ has the following jumps:

$$
T_{+}(x)=T_{-}(x) J_{T}(x), \quad \text { for } \quad x \in \mathbb{R} \backslash\{t\},
$$

where

$$
J_{T}(x)=\left(\begin{array}{cc}
e^{-n\left(g_{+}(x)-g_{-}(x)\right)} & |x-t|^{\alpha} e^{n\left(g_{+}(x)+g_{-}(x)+\ell-V(x)\right)} \\
0 & e^{n\left(g_{+}(x)-g_{-}(x)\right)}
\end{array}\right), \quad \text { for } \quad x \in \mathbb{R} \backslash\{t\} .
$$

(c) As $z \rightarrow \infty$, we have $T(z)=I+\mathcal{O}\left(z^{-1}\right)$.

(d) As $z$ tends to $t$, the behaviour of $T$ is

$$
\begin{aligned}
T(z) & =\left(\begin{array}{ll}
\mathcal{O}(1) & \mathcal{O}(\log (z-t)) \\
\mathcal{O}(1) & \mathcal{O}(\log (z-t))
\end{array}\right), \quad \text { if } \quad \alpha=0, \\
T(z) & =\left(\begin{array}{ll}
\mathcal{O}(1) & \mathcal{O}(1)+\mathcal{O}\left((z-t)^{\alpha}\right) \\
\mathcal{O}(1) & \mathcal{O}(1)+\mathcal{O}\left((z-t)^{\alpha}\right)
\end{array}\right), \quad \text { if } \quad \alpha \neq 0 .
\end{aligned}
$$

From now on, we will separate the analysis into two parts, depending on whether $\lambda \geq \lambda_{c}(t)$ or $0<\lambda<\lambda_{c}(t)$.

\section{RH analysis for $\lambda \geq \lambda_{c}(t)$}

In this section, $t$ lies in a compact subset of $(-1, \infty)$ and $\lambda$ lies in $\left[\lambda_{c}(t), \infty\right]$ as $n \rightarrow \infty$. The parameter $\lambda$ is not necessarily bounded, and the case $\lambda=+\infty$ (i.e., $s=0$ ) is also included in this analysis. First, we express the jumps for $T$ in terms of $\xi(z)$, which is defined by

$$
\xi(z)=-\pi \int_{\bar{c}}^{z} \tilde{\rho}(w) \mathrm{d} w
$$


where the path of integration lies in $\mathbb{C} \backslash(-\infty, \bar{c})$ and $\tilde{\rho}$ is given by

$$
\tilde{\rho}(z)=\frac{2}{\pi}(z-\bar{b}) \frac{\sqrt{z-\bar{c}}}{\sqrt{z-t}}
$$

where in the above expression the principal branch is chosen for each square root. The function $\xi$ is analytic in $\mathbb{C} \backslash(-\infty, \bar{c}]$, and since $\tilde{\rho}_{ \pm}(x)= \pm i \rho(x)$ for $x \in \mathcal{S}=[t, \bar{c}]$, we have

$$
2 \xi_{ \pm}(x)= \pm\left(g_{+}(x)-g_{-}(x)\right)=2 g_{ \pm}(x)+\ell-2 x^{2}, \quad x \in \mathcal{S},
$$

where we have used (4.7), (4.24) and (4.26). Thus the function $\xi(z)-g(z)$ has no jump along $\mathcal{S}$ and can be analytically continued on $\mathbb{C}$. This implies the following relation

$$
\xi(z)=g(z)+\frac{\ell}{2}-z^{2}, \quad \text { for all } \quad z \in \mathbb{C} \backslash(-\infty, \bar{c}) .
$$

The jump matrix $J_{T}$ can be rewritten in terms of $\xi$ as follows:

$$
J_{T}(x)= \begin{cases}\left(\begin{array}{cc}
1 & |x-t|^{\alpha} e^{n\left(\xi_{+}(x)+\xi_{-}(x)-\lambda\right)} \\
0 & 1
\end{array}\right), & \text { if } x<t, \\
\left(\begin{array}{cc}
e^{-2 n \xi_{+}(x)} & |x-t|^{\alpha} \\
0 & e^{2 n \xi+(x)}
\end{array}\right), & \text { if } t<x<\bar{c}, \\
\left(\begin{array}{cc}
1 & |x-t|^{\alpha} e^{2 n \xi(x)} \\
0 & 1
\end{array}\right), & \text { if } \bar{c}<x .\end{cases}
$$

As $\xi(z)$ appears in the jumps for $T$ (and in the subsequent transformations), it will be useful for us to make the following observations. From (4.8) together with Proposition 4.3, and from (4.24) and (5.4), we have that

$$
\begin{aligned}
& \xi(x)<0, \quad \text { for } \quad x>\bar{c}, \\
& \xi_{+}(x)+\xi_{-}(x)-\lambda<0, \quad \text { for } \quad x<t,
\end{aligned}
$$

except if $\lambda=\lambda_{c}(t)$ and $x=\bar{b}$, in which case (5.7) becomes an equality. Also, if $x \in(t, \bar{c})$, from the definition $(5.1)$ we have $\xi_{ \pm}(x) \in \pm i \mathbb{R}^{+}$and by Cauchy-Riemann equations we have

$$
\begin{aligned}
& \partial_{x} \Im \xi_{+}(x)=-\pi \rho(x)=-\left.\partial_{y} \Re \xi(x+i y)\right|_{y=0}, \\
& \partial_{x} \Im \xi_{-}(x)=\pi \rho(x)=\left.\partial_{y} \Re \xi(x-i y)\right|_{y=0} .
\end{aligned}
$$

In particular, this implies that there exists an open neighbourhood $W$ of $(t, \bar{c})$ such that we have

$$
\Re \xi(z)>0, \quad \text { for } \quad z \in W \backslash(t, \bar{c}) .
$$

\subsection{Second transformation: $T \mapsto S$}

We will use the following factorization of $J_{T}(x)$ for $x \in \mathcal{S}$

$$
\begin{aligned}
\left(\begin{array}{cc}
e^{-2 n \xi_{+}(x)} & |x-t|^{\alpha} \\
0 & e^{-2 n \xi_{-}(x)}
\end{array}\right)= & \left(\begin{array}{cc}
1 & 0 \\
|x-t|^{-\alpha} e^{-2 n \xi_{-}(x)} & 1
\end{array}\right) \\
& \times\left(\begin{array}{cc}
0 & |x-t|^{\alpha} \\
-|x-t|^{-\alpha} & 0
\end{array}\right)\left(\begin{array}{cc}
1 & 0 \\
|x-t|^{-\alpha} e^{-2 n \xi_{+}(x)} & 1
\end{array}\right) .
\end{aligned}
$$

We open the lenses with $\gamma_{+}$and $\gamma_{-}$around $S$ as illustrated in Fig. 1, such that $\gamma_{+} \cup \gamma_{-} \subset W$ 


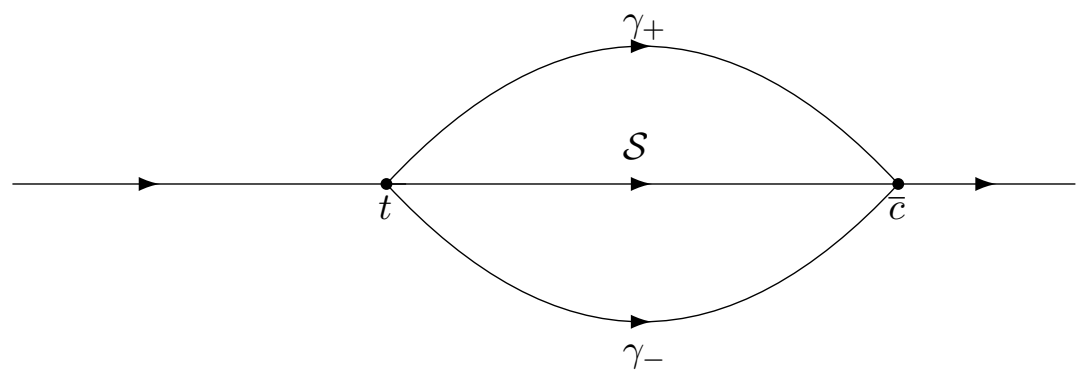

Figure 1. Jump contour for $S$.

and we define

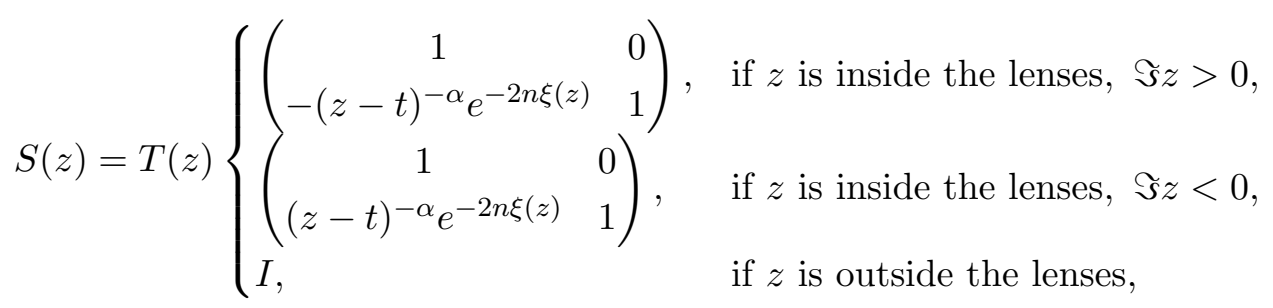

where the principal branch is taken for $(z-t)^{-\alpha} . S$ satisfies the following RH problem.

\section{RH problem for $S$}

(a) $S: \mathbb{C} \backslash\left(\mathbb{R} \cup \gamma_{+} \cup \gamma_{-}\right) \rightarrow \mathbb{C}^{2 \times 2}$ is analytic, where $\gamma_{+}$and $\gamma_{-}$are shown in Fig. 1 .

(b) $S$ has the following jumps:

$$
\begin{aligned}
& S_{+}(z)=S_{-}(z)\left(\begin{array}{cc}
1 & |z-t|^{\alpha} e^{n\left(\xi_{+}(z)+\xi_{-}(z)-\lambda\right)} \\
0 & 1
\end{array}\right), \quad \text { if } \quad z<t, \\
& S_{+}(z)=S_{-}(z)\left(\begin{array}{cc}
1 & |z-t|^{\alpha} e^{2 n \xi(z)} \\
0 & 1
\end{array}\right), \quad \text { if } \quad \bar{c}<z, \\
& S_{+}(z)=S_{-}(z)\left(\begin{array}{cc}
0 & |z-t|^{\alpha} \\
-|z-t|^{-\alpha} & 0
\end{array}\right), \quad \text { if } t<z<\bar{c}, \\
& S_{+}(z)=S_{-}(z)\left(\begin{array}{cc}
1 & 0 \\
(z-t)^{-\alpha} e^{-2 n \xi(z)} & 1
\end{array}\right), \quad \text { if } \quad z \in \gamma_{+} \cup \gamma_{-} .
\end{aligned}
$$

(c) As $z \rightarrow \infty$, we have $S(z)=I+\mathcal{O}\left(z^{-1}\right)$.

(d) As $z$ tends to $t$, we have

$$
\begin{aligned}
& S(z)=\left\{\begin{array}{ll}
\left(\begin{array}{ll}
\mathcal{O}(1) & \mathcal{O}(\log (z-t)) \\
\mathcal{O}(1) & \mathcal{O}(\log (z-t))
\end{array}\right), \quad z \text { outside the lenses, } \\
\mathcal{O}(\log (z-t)) & \mathcal{O}(\log (z-t)) \\
\mathcal{O}(\log (z-t)) & \mathcal{O}(\log (z-t))
\end{array}\right), \quad z \text { inside the lenses, } \quad \alpha=0, \\
& S(z)=\left\{\begin{array}{ll}
\left(\begin{array}{ll}
\mathcal{O}(1) & \mathcal{O}(1) \\
\mathcal{O}(1) & \mathcal{O}(1)
\end{array}\right), & z \text { outside the lenses, } \\
\mathcal{O}\left((z-t)^{-\alpha}\right) & \mathcal{O}(1) \\
\mathcal{O}\left((z-t)^{-\alpha}\right) & \mathcal{O}(1)
\end{array}\right), \quad z \text { inside the lenses, } \quad \alpha>0, \\
& S(z)=\left(\begin{array}{ll}
\mathcal{O}(1) & \mathcal{O}\left((z-t)^{\alpha}\right) \\
\mathcal{O}(1) & \mathcal{O}\left((z-t)^{\alpha}\right)
\end{array}\right), \quad \text { if } \quad \alpha<0 .
\end{aligned}
$$

As $z$ tends to $\bar{c}$, we have $S(z)=\mathcal{O}(1)$. 
From (5.6), (5.7) and (5.8), we have that the jumps for $S(z)$ on the boundary of the lenses $\gamma_{+} \cup \gamma_{-}$ tend to the identity matrix as $n \rightarrow \infty$, and that the $(1,2)$ entry of the jumps on $\mathbb{R} \backslash([t, \bar{c}] \cup\{\bar{b}\})$ tends to 0 as $n \rightarrow \infty$. This convergence is slower when $z$ approaches $t$ and $\bar{c}$, and also when $z$ approaches $\bar{b}$ if $\lambda=\lambda_{c}(t)$. The jump for $S$ on $(t, \bar{c})$ is independent of $n$ and different from the identity matrix.

\subsection{Global parametrix}

Ignoring the exponentially small terms as $n \rightarrow \infty$ in the jumps of $S$ and a small neighbourhood

of $\bar{b}, t$ and $\bar{c}$, we are left to consider the following RH problem, whose solution $P^{(\infty)}$ is a good approximation of $S$ away from a neighbourhood of $\bar{b}, t$ and $\bar{c}$.

\section{RH problem for $\boldsymbol{P}^{(\infty)}$}

(a) $P^{(\infty)}: \mathbb{C} \backslash[t, \bar{c}] \rightarrow \mathbb{C}^{2 \times 2}$ is analytic.

(b) $P^{(\infty)}$ has the following jumps:

$$
P_{+}^{(\infty)}(z)=P_{-}^{(\infty)}(z)\left(\begin{array}{cc}
0 & |z-t|^{\alpha} \\
-|z-t|^{-\alpha} & 0
\end{array}\right), \quad \text { if } \quad t<z<\bar{c} .
$$

(c) As $z \rightarrow \infty$, we have $P^{(\infty)}(z)=I+\mathcal{O}\left(z^{-1}\right)$.

(d) As $z$ tends to $t$, we have $P^{(\infty)}(z)=\mathcal{O}\left((z-t)^{-1 / 4}\right)(z-t)^{-\frac{\alpha}{2} \sigma_{3}}$.

As $z$ tends to $\bar{c}$, we have $P^{(\infty)}(z)=\mathcal{O}\left((z-\bar{c})^{-1 / 4}\right)$.

The construction of the solution of the above $\mathrm{RH}$ problem is now standard, and can be done similarly as in $[2,29,30]$. We define $\beta(z)=\sqrt[4]{\frac{z-t}{z-\bar{c}}}$, analytic on $\mathbb{C} \backslash[t, \bar{c}]$ and such that $\beta(z) \sim 1$ as $z \rightarrow \infty$. It can be checked that the unique solution of the above $\mathrm{RH}$ problem is given by

$$
\begin{aligned}
P^{(\infty)}(z)= & \frac{1}{2}\left(\frac{4}{\bar{c}-t}\right)^{-\frac{\alpha}{2} \sigma_{3}}\left(\begin{array}{cc}
\beta(z)+\beta^{-1}(z) & i\left(\beta(z)-\beta^{-1}(z)\right) \\
-i\left(\beta(z)-\beta^{-1}(z)\right) & \beta(z)+\beta^{-1}(z)
\end{array}\right) \\
& \times \varphi\left(\frac{2}{\bar{c}-t}\left(z-\frac{\bar{c}+t}{2}\right)\right)^{\frac{\alpha}{2} \sigma_{3}}(z-t)^{-\frac{\alpha}{2} \sigma_{3}},
\end{aligned}
$$

where $\varphi(z)=z+\sqrt{z^{2}-1}$ is analytic in $\mathbb{C} \backslash[-1,1]$ and such that $\varphi(z) \sim 2 z$ as $z \rightarrow \infty$. We will later need the following expansion as $z \rightarrow \infty$ :

$$
P_{11}^{(\infty)}(z)=1-\frac{\alpha(\bar{c}-t)}{4 z}+\mathcal{O}\left(z^{-2}\right)
$$

\subsection{Local parametrix near $t$}

Note that the assumption at the beginning of the section, i.e., that $t$ lies in a compact subset of $(-1, \infty)$ and $\lambda \geq \lambda_{c}(t)$ as $n \rightarrow \infty$, implies from Proposition 4.3 that there exists a constant $\delta>0$ independent of $n$ such that

$$
\delta<\min (t-\bar{b}, \bar{c}-t) .
$$

Inside a disk $D_{t}$ around $t$, of radius fixed but smaller than $\delta / 3$, we want the local parametrix $P$ to satisfy exactly the same jumps as $S$ and to have the same behaviour as $S$ near $t$. Furthermore, the local parametrix $P$ should be close to the global parametrix on the boundary of the disk. 


\section{RH problem for $P$}

(a) $P: D_{t} \backslash\left(\mathbb{R} \cup \gamma_{+} \cup \gamma_{-}\right) \rightarrow \mathbb{C}^{2 \times 2}$ is analytic.

(b) $P$ has the following jumps:

$$
\begin{aligned}
& P_{+}(z)=P_{-}(z)\left(\begin{array}{cc}
1 & |z-t|^{\alpha} e^{n\left(\xi_{+}(z)+\xi_{-}(z)-\lambda\right)} \\
0 & 1
\end{array}\right), \quad \text { if } \quad z \in(-\infty, t) \cap D_{t}, \\
& P_{+}(z)=P_{-}(z)\left(\begin{array}{cc}
0 & |z-t|^{\alpha} \\
-|z-t|^{-\alpha} & 0
\end{array}\right), \quad \text { if } \quad z \in(t, \infty) \cap D_{t}, \\
& P_{+}(z)=P_{-}(z)\left(\begin{array}{cc}
1 & 0 \\
(z-t)^{-\alpha} e^{-2 n \xi(z)} & 1
\end{array}\right), \quad \text { if } \quad z \in\left(\gamma_{+} \cup \gamma_{-}\right) \cap D_{t} .
\end{aligned}
$$

(c) As $n \rightarrow \infty$, we have $P(z)=\left(I+\mathcal{O}\left(n^{-1}\right)\right) P^{(\infty)}(z)$ uniformly for $z \in \partial D_{t}$.

(d) As $z$ tends to $t$, we have $S(z) P(z)^{-1}=\mathcal{O}(1)$.

The construction of a local parametrix associated with a FH singularity has been studied in [27] when the singularity is a pure jump, and then in [24] and [17] for the general case, and involves hypergeometric functions. On the other hand, the construction of a local parametrix associated to a pure root-type FH singularity involves Bessel functions [30]. In the present case, we are in a presence of a FH singularity of both root-type and jump-type, but the significant difference is that the parameter $s$ (which parametrizes the jump) is exponentially small as $n \rightarrow \infty$. The solution of the present local parametrix will be expressed in terms of Bessel functions, exactly as for a pure root-type singularity. Nevertheless, as the $(1,2)$ element of the jump matrix for $P$ in (5.13) is not zero (if $\lambda \neq+\infty$, i.e., $s \neq 0$ ), the construction of the solution of the above $\mathrm{RH}$ problem is not standard. It was done in [10] for the case $\alpha=0$. We generalize here the construction for a general $\alpha>-1$.

We will need a modified version of the Bessel model $\mathrm{RH}$ problem $P_{\mathrm{Be}}$, which is presented in Appendix B. We search for a matrix function $\widehat{P}_{\mathrm{Be}}$ which satisfies the same jumps as $P_{\mathrm{Be}}$, see (B.1), and an extra jump on $\mathbb{R}^{+}$given by

$$
\widehat{P}_{\mathrm{Be}}(z)_{+}=\widehat{P}_{\mathrm{Be}}(z)_{-}\left(\begin{array}{cc}
1 & e^{-\lambda n} \\
0 & 1
\end{array}\right), \quad z \in(0, \infty),
$$

where the orientation of $(0, \infty)$ is taken from 0 to $\infty$.

\section{Modified Bessel model RH problem}

We define

$$
F(z)=P_{\mathrm{Be}}(z) K(z)^{-1}\left(\begin{array}{cc}
1 & -h(z) \\
0 & 1
\end{array}\right) z^{-\frac{\alpha}{2} \sigma_{3}}
$$

where

$$
K(z)=\left\{\begin{array}{ll}
I, & |\arg z|<\frac{2 \pi}{3}, \\
\left(\begin{array}{cc}
1 & 0 \\
-e^{\pi i \alpha} & 1
\end{array}\right), & \frac{2 \pi}{3}<\arg z<\pi, \\
\left(\begin{array}{cc}
1 & 0 \\
e^{-\pi i \alpha} & 1
\end{array}\right), & -\pi<\arg z<-\frac{2 \pi}{3},
\end{array} \quad h(z)= \begin{cases}\frac{1}{2 i \sin (\pi \alpha)}, & \text { if } \alpha \notin \mathbb{N}, \\
\frac{(-1)^{\alpha}}{2 \pi i} \log z, & \text { if } \alpha \in \mathbb{N} .\end{cases}\right.
$$


From the jumps for $P_{\mathrm{Be}}$, given by (B.1), it can be checked that $F$ has no jumps at all on $\mathbb{C}$. Also, the behaviour of $P_{\mathrm{Be}}(z)$ as $z \rightarrow 0$, given by (B.3), implies that 0 is a removable singularity of $F$, and thus $F$ is an entire function. We define $\widehat{P}_{\mathrm{Be}}$ by

$$
\widehat{P}_{\mathrm{Be}}(z)=(I+A(z)) P_{\mathrm{Be}}(z),
$$

where

$$
A(z)=-e^{-\lambda n} h(-z)(-z)^{\alpha} F(z)\left(\begin{array}{cc}
0 & 1 \\
0 & 0
\end{array}\right) F^{-1}(z),
$$

and if $\alpha \notin \mathbb{Z},(-z)^{\alpha}$ is chosen with a branch cut on $[0, \infty)$ such that $(-z)^{\alpha}>0$ for $z<0$. Since $F$ is entire, $A$ is analytic on $\mathbb{C} \backslash \mathbb{R}^{+}$. Therefore, it can be checked that $\widehat{P}_{\mathrm{Be}}$ is the solution of the following $\mathrm{RH}$ problem.

\section{RH problem for $\widehat{P}_{\mathrm{Be}}$}

(a) $\widehat{P}_{\mathrm{Be}}: \mathbb{C} \backslash\left(\Sigma_{B} \cup(0, \infty)\right) \rightarrow \mathbb{C}^{2 \times 2}$ is analytic, where the orientation of $(0, \infty)$ is from 0 towards $\infty$ and $\Sigma_{B}$ is the jump contour for $P_{\text {Be }}$, shown in Fig. 7 .

(b) $\widehat{P}_{\mathrm{Be}}$ satisfies the jump conditions

$$
\begin{aligned}
& \widehat{P}_{\mathrm{Be},+}(z)=\widehat{P}_{\mathrm{Be},-}(z)\left(\begin{array}{cc}
0 & 1 \\
-1 & 0
\end{array}\right), \quad z \in \mathbb{R}^{-}, \\
& \widehat{P}_{\mathrm{Be},+}(z)=\widehat{P}_{\mathrm{Be},-}(z)\left(\begin{array}{cc}
1 & 0 \\
e^{\pi i \alpha} & 1
\end{array}\right), \quad z \in e^{\frac{2 \pi i}{3}} \mathbb{R}^{+}, \\
& \widehat{P}_{\mathrm{Be},+}(z)=\widehat{P}_{\mathrm{Be},-}(z)\left(\begin{array}{cc}
1 & 0 \\
e^{-\pi i \alpha} & 1
\end{array}\right), \quad z \in e^{-\frac{2 \pi i}{3}} \mathbb{R}^{+}, \\
& \widehat{P}_{\mathrm{Be},+}(z)=\widehat{P}_{\mathrm{Be},-}(z)\left(\begin{array}{cc}
1 & e^{-\lambda n} \\
0 & 1
\end{array}\right), \quad z \in \mathbb{R}^{+} .
\end{aligned}
$$

(c) As $z \rightarrow \infty, z \notin \Sigma_{B} \cup(0, \infty)$, we have

$$
\widehat{P}_{\mathrm{Be}}(z)=(I+A(z))\left(2 \pi z^{\frac{1}{2}}\right)^{-\frac{\sigma_{3}}{2}} N\left(I+\mathcal{O}\left(z^{-\frac{1}{2}}\right)\right) e^{2 z^{\frac{1}{2}} \sigma_{3}},
$$

where $N=\frac{1}{\sqrt{2}}\left(\begin{array}{ll}1 & i \\ i & 1\end{array}\right)$.

(d) As $z$ tends to 0 , the behaviour of $\widehat{P}_{\mathrm{Be}}(z)$ is

$$
\begin{aligned}
& P_{\mathrm{Be}}(z)^{-1} \widehat{P}_{\mathrm{Be}}(z)=\mathcal{O}(\log z), \quad \text { if } \quad \alpha \in \mathbb{N}, \\
& P_{\mathrm{Be}}(z)^{-1} \widehat{P}_{\mathrm{Be}}(z)=\mathcal{O}(1), \quad \text { if } \quad \alpha \notin \mathbb{N} .
\end{aligned}
$$

\section{Construction of the local parametrix}

We consider the function

$$
f(z)=-\frac{1}{4} \tilde{\xi}(z)^{2}, \quad \text { where } \quad \tilde{\xi}(z)= \begin{cases}\xi(z)-\xi_{+}(t), & \text { if } \Im z>0 \\ \xi(z)-\xi_{-}(t), & \text { if } \Im z<0 .\end{cases}
$$

This a conformal map from $D_{t}$ to a neighbourhood of 0 , and as $z \rightarrow t$, we have

$$
f(z)=k_{1}^{2}(z-t)(1+\mathcal{O}(z-t)), \quad \text { where } \quad k_{1}=2(t-\bar{b}) \sqrt{\bar{c}-t} .
$$


To construct the solution $P$ of the above RH problem, it is important to note that $A\left(-n^{2} f(z)\right)$ remains small as $n \rightarrow \infty$ uniformly for $z \in \partial D_{t}$. More precisely, from (5.14) and (B.2), as $n \rightarrow \infty$ we have

$$
F\left(-n^{2} f(z)\right)=\mathcal{O}(\log n) \mathcal{O}\left(n^{|\alpha|}\right) \mathcal{O}\left(P_{\mathrm{Be}}\left(-n^{2} f(z)\right)\right)=\mathcal{O}\left(e^{(d+\epsilon) n}\right),
$$

where $\epsilon>0$ can be chosen arbitrary small but fixed, and $d=\max _{z \in \partial D_{t}}|2 \sqrt{-f(z)}|$. Similarly, we have the estimate $F^{-1}\left(-n^{2} f(z)\right)=\mathcal{O}\left(e^{(d+\epsilon) n}\right)$. We choose the radius of $D_{t}$ fixed but sufficiently small such that $d<\frac{\lambda_{c}(t)}{3} \leq \frac{\lambda}{3}$. This implies from (5.15) that $A\left(-n^{2} f(z)\right)=\mathcal{O}\left(e^{-\frac{\lambda}{3} n}\right)$ as $n \rightarrow \infty$, uniformly for $z \in \partial D_{t}$. The local parametrix is given by

$$
P(z)=E(z) \sigma_{3} \widehat{P}_{\mathrm{Be}}\left(-n^{2} f(z)\right) \sigma_{3} e^{-n \xi(z) \sigma_{3}} e^{\frac{\pi i \alpha}{2} \tilde{\theta}(z) \sigma_{3}}(z-t)^{-\frac{\alpha}{2} \sigma_{3}},
$$

where

$$
\tilde{\theta}(z)= \begin{cases}+1, & \text { if } \Im z>0 \\ -1, & \text { if } \Im z<0,\end{cases}
$$

and the function $E(z)$ is defined for $z \in D_{t}$ by

$$
E(z)=(-1)^{n} P^{(\infty)}(z)(z-t)^{\frac{\alpha}{2} \sigma_{3}} e^{-\frac{\pi i \alpha}{2} \tilde{\theta}(z) \sigma_{3}} N\left(2 \pi n(-f(z))^{1 / 2}\right)^{\sigma_{3} / 2} .
$$

It can be checked directly from the jumps for $P^{(\infty)}$ (5.10) that $E$ has no jump at all in $D_{t}$. Furthermore, from the behaviour of $P^{(\infty)}(z)$ near $t$ and from (5.17), one has $E(z)=\mathcal{O}\left((z-t)^{-\frac{1}{2}}\right)$ as $z \rightarrow t$. Thus, $t$ is a removable singularity of $E$ and $E$ is analytic in the whole disk $D_{t}$. Since $A\left(-n^{2} f(z)\right)$ is exponentially small in $n$ uniformly for $z \in \partial D_{t}$, it doesn't contribute to the $n^{-1}$ term in the condition (c) of the $\mathrm{RH}$ problem for $P$. Using the large $\zeta$ asymptotics for the Bessel model RH problem given by (B.2), we obtain as $n \rightarrow \infty$ that

$$
\begin{aligned}
P(z) P^{\infty}(z)^{-1}= & I+\frac{1}{n(-f(z))^{1 / 2}} P^{(\infty)}(z)(z-t)^{\frac{\alpha}{2} \sigma_{3}} e^{-\frac{\pi i \alpha}{2} \tilde{\theta}(z) \sigma_{3}} \sigma_{3} B_{1} \sigma_{3} \\
& \times e^{\frac{\pi i \alpha}{2} \tilde{\theta}(z) \sigma_{3}}(z-t)^{-\frac{\alpha}{2} \sigma_{3}} P^{(\infty)}(z)^{-1}+\mathcal{O}\left(n^{-2}\right),
\end{aligned}
$$

uniformly for $z \in \partial D_{t}$, where $B_{1}=\frac{1}{16}\left(\begin{array}{cc}-\left(1+4 \alpha^{2}\right) & -2 i \\ -2 i & 1+4 \alpha^{2}\end{array}\right)$.

\subsection{Local parametrix near $\bar{c}$}

Inside a disk $D_{\bar{c}}$ around $\bar{c}$, of radius fixed but smaller than $\delta / 3$, we want the local parametrix $P$ to satisfy the following $\mathrm{RH}$ problem.

\section{RH problem for $P$}

(a) $P: D_{\bar{c}} \backslash\left(\mathbb{R} \cup \gamma_{+} \cup \gamma_{-}\right) \rightarrow \mathbb{C}^{2 \times 2}$ is analytic.

(b) $P$ has the following jumps:

$$
\begin{aligned}
& P_{+}(z)=P_{-}(z)\left(\begin{array}{cc}
0 & |z-t|^{\alpha} \\
-|z-t|^{-\alpha} & 0
\end{array}\right), \quad \text { if } \quad z \in(-\infty, \bar{c}) \cap D_{\bar{c}}, \\
& P_{+}(z)=P_{-}(z)\left(\begin{array}{cc}
1 & |z-t|^{\alpha} e^{2 n \xi(z)} \\
0 & 1
\end{array}\right), \quad \text { if } \quad z \in(\bar{c}, \infty) \cap D_{\bar{c}}, \\
& P_{+}(z)=P_{-}(z)\left(\begin{array}{cc}
1 & 0 \\
(z-t)^{-\alpha} e^{-2 n \xi(z)} & 1
\end{array}\right), \quad \text { if } \quad z \in\left(\gamma_{+} \cup \gamma_{-}\right) \cap D_{\bar{c}} .
\end{aligned}
$$


(c) As $n \rightarrow \infty$, we have $P(z)=\left(I+\mathcal{O}\left(n^{-1}\right)\right) P^{(\infty)}(z)$ uniformly for $z \in \partial D_{\bar{c}}$.

(d) As $z$ tends to $\bar{c}$, we have $P(z)=\mathcal{O}(1)$.

The solution $P$ of the above RH problem is standard [18] and can be constructed in terms of Airy functions and the associated Airy model RH problem, whose solution is denoted $P_{\mathrm{Ai}}$ and is presented in Appendix A. Let us first define the function

$$
f(z)=\left(-\frac{3}{2} \xi(z)\right)^{2 / 3}
$$

This is a conformal map from $D_{\bar{c}}$ to a neighbourhood of 0 , and as $z \rightarrow \bar{c}$ we have

$$
f(z)=k_{2}^{2 / 3}(z-\bar{c})\left[1+\frac{2}{5} k_{3}(z-\bar{c})+\mathcal{O}\left((z-\bar{c})^{2}\right)\right],
$$

with

$$
k_{2}=2 \frac{\bar{c}-\bar{b}}{\sqrt{\bar{c}-t}}, \quad k_{3}=\frac{1}{\bar{c}-\bar{b}}-\frac{1}{2(\bar{c}-t)} .
$$

It can be verified that

$$
P(z)=E(z) P_{\mathrm{Ai}}\left(n^{2 / 3} f(z)\right) e^{-n \xi(z) \sigma_{3}}(z-t)^{-\frac{\alpha}{2} \sigma_{3}},
$$

satisfies the above $\mathrm{RH}$ problem, where

$$
E(z)=P^{(\infty)}(z)(z-t)^{\frac{\alpha}{2} \sigma_{3}} N^{-1} f(z)^{\frac{\sigma_{3}}{4}} n^{\frac{\sigma_{3}}{6}} .
$$

Again, one can show that $E$ has no jump at all inside $D_{\bar{c}}$ and has a removable singularity at $\bar{c}$, and therefore $E$ is analytic in the whole disk $D_{\bar{c}}$. We will also need explicitly the first term in the large $n$ expansion of $P(z) P^{(\infty)}(z)^{-1}$ on $\partial D_{\bar{c}}$. As $n \rightarrow \infty$, by (A.1) we have

$$
\begin{aligned}
P(z) P^{(\infty)}(z)^{-1}= & I+\frac{1}{n f(z)^{3 / 2}} P^{(\infty)}(z)(z-t)^{\frac{\alpha}{2} \sigma_{3}} A_{1}(z-t)^{-\frac{\alpha}{2} \sigma_{3}} P^{(\infty)}(z)^{-1} \\
& +\mathcal{O}\left(n^{-2}\right) .
\end{aligned}
$$

uniformly for $z \in \partial D_{\bar{c}}$, and where $A_{1}=\frac{1}{8}\left(\begin{array}{cc}\frac{1}{6} & i \\ i & -\frac{1}{6}\end{array}\right)$,

\subsection{Local parametrix near $\bar{b}$}

The local parametrix $P$ in a fixed disk $D_{\bar{b}}$ around $\bar{b}$ can be constructed explicitly. The construction is similar to the one done in [10] and it is valid for every $\lambda \geq \lambda_{c}$ but is only needed for $\lambda$ close to $\lambda_{c}$.

\section{RH problem for $P$}

(a) $P: D_{\bar{b}} \backslash \mathbb{R} \rightarrow \mathbb{C}^{2 \times 2}$ is analytic.

(b) $P$ has the following jumps:

$$
P_{+}(z)=P_{-}(z)\left(\begin{array}{cc}
1 & |z-t|^{\alpha} e^{n\left(\xi_{+}(z)+\xi_{-}(z)-\lambda\right)} \\
0 & 1
\end{array}\right), \quad \text { if } \quad z \in \mathbb{R} \cap D_{\bar{b}} .
$$

(c) As $n \rightarrow \infty$, we have $P(z)=(I+o(1)) P^{(\infty)}(z)$ uniformly for $z \in \partial D_{\bar{b}}$. 


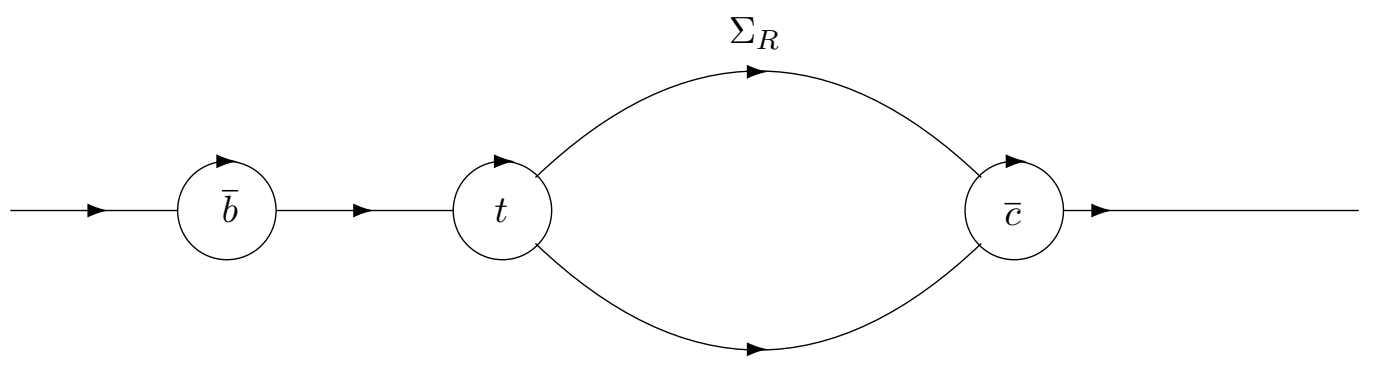

Figure 2. Jump contours for the RH problem for $R$. The circles are oriented in clockwise direction.

Note that for $z \in D_{\bar{b}}$, we have

$$
\phi(z)=\xi_{+}(z)+\xi_{-}(z)=2 \pi \int_{z}^{t} \tilde{\rho}(w) \mathrm{d} w,
$$

and $\phi$ is analytic in $D_{\bar{b}}$. The unique solution of the above RH problem is given by

$$
P(z)=P^{(\infty)}(z)\left(\begin{array}{cc}
1 & l(z) \\
0 & 1,
\end{array}\right)
$$

where

$$
l(z)=\frac{(z-t)^{\alpha} e^{-\pi i \alpha \tilde{\theta}(z)} e^{-n\left(\lambda-\lambda_{c}\right)}}{2 \pi i} \int_{\mathbb{R} \cap D_{\bar{b}}} \frac{e^{n\left(\phi(x)-\lambda_{c}\right)}}{x-z} \mathrm{~d} x .
$$

From (5.7) and (5.24), we have $\phi(\bar{b})=\lambda_{c}, \phi^{\prime}(\bar{b})=0$ and $\phi^{\prime \prime}(\bar{b})<0$. Thus, as $n \rightarrow \infty$ we have $l(z)=\mathcal{O}\left(n^{-1 / 2} e^{-n\left(\lambda-\lambda_{c}\right)}\right)$ and

$$
P(z)=\left(I+\mathcal{O}\left(n^{-1 / 2} e^{-n\left(\lambda-\lambda_{c}\right)}\right)\right) P^{(\infty)}(z), \quad \text { uniformly for } \quad z \in \partial D_{\bar{b}} .
$$

\subsection{Small norm RH problem}

The final transformation of the $\mathrm{RH}$ analysis is given by

$$
R(z)= \begin{cases}S(z) P^{(\infty)}(z)^{-1}, & z \in \mathbb{C} \backslash\left(\overline{D_{t}} \cup \overline{D_{\bar{c}}} \cup \overline{D_{\bar{b}}}\right), \\ S(z) P(z)^{-1}, & z \in D_{t} \cup D_{\bar{c}} \cup D_{\bar{b}} .\end{cases}
$$

Since $P$ has exactly the same jumps as $S$ inside $D_{t} \cup D_{\bar{c}} \cup D_{\bar{b}}$, and the same behaviour near $t$ and $\bar{c}, R$ has no jumps and is analytic inside these disks, except possibly at $\bar{b}, \bar{c}$ and $t$. From the $\mathrm{RH}$ problem for $S$, and from the local parametrices around $\bar{b}$ and $\bar{c}$, we have that $S(z)$ and $P(z)$ are bounded as $z \rightarrow \bar{b}$ and as $z \rightarrow \bar{c}$. From (5.9), (5.19) and (B.3), as $z \rightarrow t$ from outside the lenses, we have

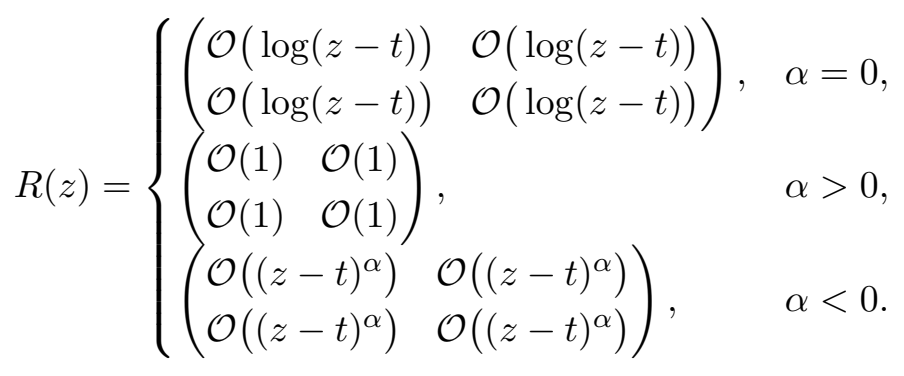

By (5.25), and since $R(z)$ is bounded as $z \rightarrow \bar{b}$ and as $z \rightarrow \bar{c}$, the three isolated singularities at $\bar{b}, \bar{c}$ and $t$ are removable. On the circles $\partial D_{t}, \partial D_{\bar{c}}$ and $\partial D_{\bar{c}}$, we choose the clockwise orientation, as shown in Fig. 2. $R$ satisfies the following $\mathrm{RH}$ problem: 


\section{RH problem for $R$}

(a) $R: \mathbb{C} \backslash \Sigma_{R} \rightarrow \mathbb{C}^{2 \times 2}$ is analytic, where the contour $\Sigma_{R}$ is shown in Fig. 2 .

(b) The jumps $J_{R}(z):=R_{-}^{-1}(z) R_{+}(z)$ satisfy the following large $n$ asymptotics for $z \in \Sigma_{R}$ :

$$
\begin{aligned}
& J_{R}(z)=I+\mathcal{O}\left(e^{-c n}\right), \quad \text { uniformly for } \quad z \in\left(\gamma_{+} \cup \gamma_{-} \cup \mathbb{R}\right) \backslash\left(\mathcal{S} \cup \overline{D_{t}} \cup \overline{D_{\bar{c}}} \cup \overline{D_{\bar{b}}}\right), \\
& J_{R}(z)=I+\mathcal{O}\left(n^{-1}\right), \quad \text { uniformly for } \quad z \in \partial D_{t} \cup \partial D_{\bar{c}}, \\
& J_{R}(z)=I+\mathcal{O}\left(n^{-1 / 2} e^{-n\left(\lambda-\lambda_{c}\right)}\right), \quad \text { uniformly for } \quad z \in \partial D_{\bar{b}},
\end{aligned}
$$

where $c>0$ is a constant.

(c) As $z \rightarrow \infty$, we have $R(z)=I+\mathcal{O}\left(z^{-1}\right)$.

From the standard theory for small-norm RH problems [18], $R$ exists for all $n$ sufficiently large and as $n \rightarrow \infty$ we have

$$
\begin{aligned}
& R(z)=I+\mathcal{O}\left(n^{-1}\right)+\mathcal{O}\left(n^{-1 / 2} e^{-n\left(\lambda-\lambda_{c}\right)}\right), \\
& R^{\prime}(z)=\mathcal{O}\left(n^{-1}\right)+\mathcal{O}\left(n^{-1 / 2} e^{-n\left(\lambda-\lambda_{c}\right)}\right),
\end{aligned}
$$

uniformly for $z \in \mathbb{C} \backslash \Sigma_{R}$, and uniformly for $t$ in compact subsets of $(-1, \infty)$ and for $\lambda \geq \lambda_{c}(t)$. In the rest of this section, we consider the case when $\lambda$ lies in a compact subset of $\left(\lambda_{c}(t), \infty\right]$ as $n \rightarrow \infty$, i.e., when $\lambda$ is bounded away from $\lambda_{c}(t)$. In this case, the jumps for $R$ on $\partial D_{\bar{b}}$ are exponentially small. On the other hand, the jumps for $R$ on $\partial D_{t} \cup \partial D_{\bar{c}}$ have a series expansion of the form

$$
J_{R}(z)=I+\sum_{j=1}^{r} J_{R}^{(j)}(z) n^{-j}+\mathcal{O}\left(n^{-r-1}\right),
$$

for any $r \in \mathbb{N}$, where $J_{R}^{(j)}(z)=\mathcal{O}(1)$ as $n \rightarrow \infty$ uniformly for $z \in \partial D_{t} \cup \partial D_{\bar{c}}$. Thus, $R$ admits a series expansion of the form

$$
R(z)=I+\sum_{j=1}^{r} R^{(j)}(z) n^{-j}+\mathcal{O}\left(n^{-r-1}\right), \quad \text { as } \quad n \rightarrow \infty
$$

for any $r \in \mathbb{N}$, where $R^{(j)}(z)=\mathcal{O}(1)$ as $n \rightarrow \infty$ uniformly for $z \in \mathbb{C} \backslash \Sigma_{R}$. By a perturbative analysis of the $\mathrm{RH}$ problem for $R$, the first term $R^{(1)}(z)$ is given by

$$
R^{(1)}(z)=\frac{1}{2 \pi i} \int_{\partial D_{t} \cup \partial D_{\bar{c}}} \frac{J_{R}^{(1)}(w)}{w-z} \mathrm{~d} w .
$$

We can evaluate this integral explicitly. When $z$ is outside the disks, we have from (5.22) and (5.23) that

$$
\begin{aligned}
& \left(\frac{4}{\bar{c}-t}\right)^{\frac{\alpha}{2} \sigma_{3}} R^{(1)}(z)\left(\frac{4}{\bar{c}-t}\right)^{-\frac{\alpha}{2} \sigma_{3}} \\
& =\frac{\sqrt{\bar{c}-t}}{z-t} \frac{4 \alpha^{2}-1}{32 k_{1}}\left(\begin{array}{cc}
1 & i \\
i & -1
\end{array}\right)+\frac{1}{(z-\bar{c})^{2}} \frac{5 \sqrt{\bar{c}-t}}{96 k_{2}}\left(\begin{array}{cc}
-1 & i \\
i & 1
\end{array}\right) \\
& +\frac{1}{z-\bar{c}} \frac{1}{64 \sqrt{\bar{c}-t} k_{2}}\left(\begin{array}{cc}
-8 \alpha^{2}+2(\bar{c}-t) k_{3}+3 & \frac{i}{3}\left(24(\alpha+2) \alpha-6(\bar{c}-t) k_{3}+19\right) \\
\frac{i}{3}\left(24(\alpha-2) \alpha-6(\bar{c}-t) k_{3}+19\right) & 8 \alpha^{2}-2(\bar{c}-t) k_{3}-3
\end{array}\right) .
\end{aligned}
$$




\section{RH analysis for $0<\lambda<\lambda_{c}(t)$}

In this section, we analyse the case when $(t, \lambda)$ is in a compact subset of

$$
\mathcal{R}=\left\{(t, \lambda): t \in(-1,1) \text { and } 0<\lambda<\lambda_{c}(t)\right\}
$$

as $n \rightarrow \infty$. We define the function

$$
\tilde{\rho}(z)=\frac{2}{\pi} \frac{\sqrt{z-c} \sqrt{z-b} \sqrt{z-a}}{\sqrt{z-t}},
$$

analytic in $\mathbb{C} \backslash([a, b] \cup[t, c])$ such that $\tilde{\rho}(z) \sim \frac{2}{\pi} z$ as $z \rightarrow+\infty$. We will adopt the same approach as in Section 5, and we will rewrite the jumps for $T$ in terms of the following two functions:

$$
\xi_{1}(z)=-\pi \int_{c}^{z} \tilde{\rho}(w) \mathrm{d} w, \quad \xi_{2}(z)=-\pi \int_{b}^{z} \tilde{\rho}(w) \mathrm{d} w .
$$

For $\xi_{1}$ the path of integration is chosen to be in $\mathbb{C} \backslash(-\infty, c)$, and for $\xi_{2}$ the path lies in $\mathbb{C} \backslash$ $((-\infty, b) \cup[t, \infty))$. Therefore, $\xi_{1}$ is analytic in $\mathbb{C} \backslash(-\infty, c)$, satisfies $\xi_{1}(z)<0$ for $z>c$ and $\xi_{2}$ is analytic in $\mathbb{C} \backslash((-\infty, b) \cup[t, \infty))$. Similarly to (5.3), note that $\xi_{1}(z)-g(z)$ satisfies

$$
\xi_{1,+}(z)-g_{+}(z)=\xi_{1,-}(z)-g_{-}(z)=\frac{\ell}{2}-z^{2}, \quad z \in(t, c) .
$$

Analytically continuing $\xi_{1}(z)-g(z)$ in $(6.2)$ for $z$ outside $(-\infty, t)$, we have

$$
\xi_{1}(z)=g(z)+\frac{\ell}{2}-z^{2}, \quad z \in \mathbb{C} \backslash(-\infty, c] .
$$

It will be useful later to notice the connection formula between $g(z), \xi_{1}(z)$ and $\xi_{2}(z)$ for $z \in(b, t)$ :

$$
g_{+}(z)+g_{-}(z)+\ell-2 z^{2}=\xi_{1,+}(z)+\xi_{1,-}(z)=2 \xi_{2}(z)+2 \pi \int_{b}^{t} \tilde{\rho}(x) \mathrm{d} x=2 \xi_{2}(z)+\lambda,
$$

where we have used (4.14), (4.2), (4.19) and (4.24). Furthermore, by (4.7) and (4.24), we have

$$
\xi_{2,+}(z)+\xi_{2,-}(z)=0=g_{+}(z)+g_{-}(z)-2 z^{2}+\ell-\lambda, \quad z \in(a, b),
$$

and by (4.19), we also have

$$
\left(\xi_{2,+}(z)+\xi_{2,-}(z)\right)^{\prime}=-2 \pi \tilde{\rho}(z)=\left(g_{+}(z)+g_{-}(z)-2 z^{2}+\ell-\lambda\right)^{\prime}, \quad z<a .
$$

Thus, we have the following identity between $\xi_{2}$ and $g$ on $(-\infty, a)$ :

$$
\xi_{2,+}(z)+\xi_{2,-}(z)=g_{+}(z)+g_{-}(z)-2 z^{2}+\ell-\lambda<0, \quad z<a .
$$

The mass of $\rho$ on the interval $(a, b)$ will play an important role later and will appear in the jumps of the subsequent RH problems, and we denote it as follows:

$$
\Omega(t, \lambda)=\int_{a}^{b} \rho(x ; t, \lambda) \mathrm{d} x .
$$

We will sometimes omit the dependence of $\Omega(t, \lambda)$ in $t$ and $\lambda$ and simply write $\Omega$ when there is no confusion. For $z \in(a, b)$, by the relation (4.26), we have this identity

$$
2 \xi_{2,+}(z)=2 \pi i \int_{z}^{b} \rho(x) \mathrm{d} x=g_{+}(z)-g_{-}(z)+2 \pi i \Omega-2 \pi i .
$$


Also, by Cauchy-Riemann equations, we can show similarly to equation (5.8) that there exists an open neighbourhood $W_{1}$ of $(t, c)$ and an open neighbourhood $W_{2}$ of $(a, b)$ such that

$$
\begin{array}{ll}
\Re \xi_{1}(z)>0, & \text { for } \quad z \in W_{1} \backslash(t, c), \\
\Re \xi_{2}(z)>0, & \text { for } \quad z \in W_{2} \backslash(a, b) .
\end{array}
$$

The jumps for $T$ can now be rewritten as

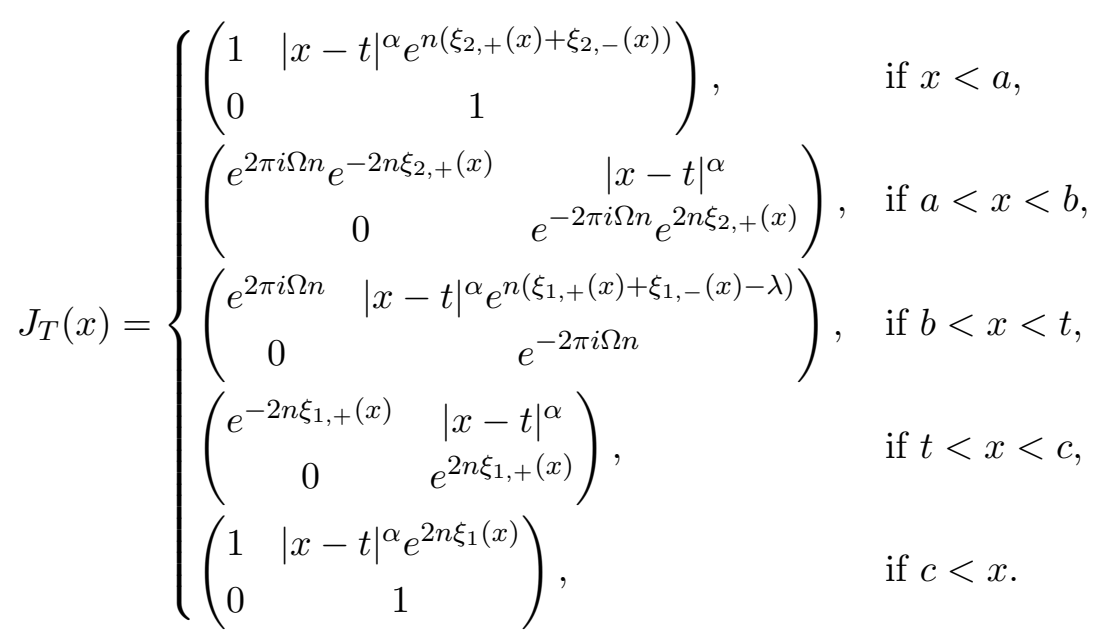

For $x \in(b, t)$, by $(6.4)$, one can also rewrite $J_{T}(x)$ as

$$
J_{T}(x)=\left(\begin{array}{cc}
e^{2 \pi i \Omega n} & |x-t|^{\alpha} e^{2 n \xi_{2}(x)} \\
0 & e^{-2 \pi i \Omega n}
\end{array}\right)
$$

\subsection{Second transformation: $T \mapsto S$}

We proceed to the opening of the lenses with $\gamma_{+}, \gamma_{-}, \tilde{\gamma}_{+}$, and $\tilde{\gamma}_{-}$as shown in Fig. 3, such that $\gamma_{+} \cup \gamma_{-} \subset W_{1}$ and $\tilde{\gamma}_{+} \cup \tilde{\gamma}_{-} \subset W_{2}$. The next transformation $S$ is defined by

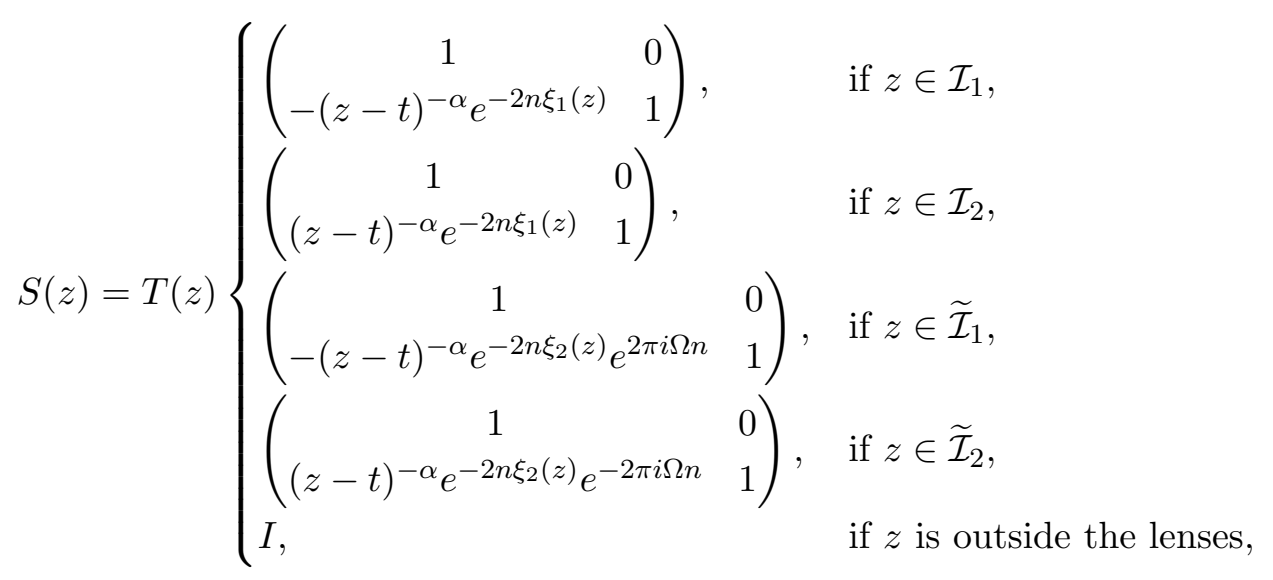

where the sectors $\mathcal{I}_{1}, \mathcal{I}_{2}, \widetilde{\mathcal{I}}_{1}$, and $\widetilde{\mathcal{I}}_{2}$ are inside the lenses and are shown in Fig. $3 . S$ satisfies the following $\mathrm{RH}$ problem.

\section{RH problem for $S$}

(a) $S: \mathbb{C} \backslash\left(\mathbb{R} \cup \gamma_{+} \cup \gamma_{-} \cup \tilde{\gamma}_{+} \cup \tilde{\gamma}_{-}\right) \rightarrow \mathbb{C}^{2 \times 2}$ is analytic, see Fig. 3 . 


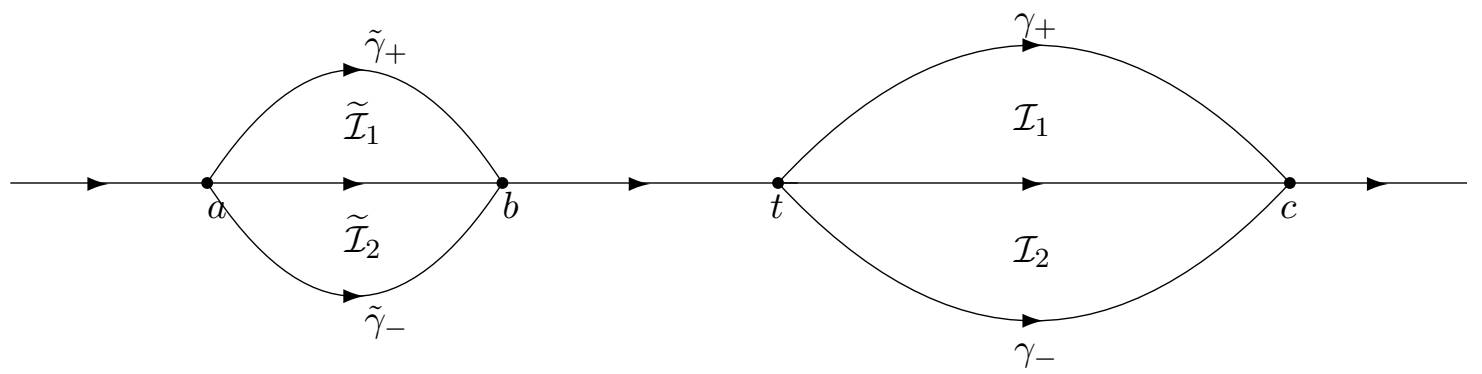

Figure 3. Jump contour for $S$.

(b) $S$ has the following jumps:

$$
\begin{aligned}
& S_{+}(z)=S_{-}(z)\left(\begin{array}{cc}
1 & |z-t|^{\alpha} e^{n\left(\xi_{2,+}(z)+\xi_{2,-}(z)\right)} \\
0 & 1
\end{array}\right), \quad \text { if } \quad z<a, \\
& S_{+}(z)=S_{-}(z)\left(\begin{array}{cc}
e^{2 \pi i \Omega n} & |z-t|^{\alpha} e^{n\left(\xi_{1,+}(z)+\xi_{1,-}(z)-\lambda\right)} \\
0 & e^{-2 \pi i \Omega n}
\end{array}\right), \quad \text { if } \quad b<z<t, \\
& S_{+}(z)=S_{-}(z)\left(\begin{array}{cc}
1 & |z-t|^{\alpha} e^{2 n \xi_{1}(z)} \\
0 & 1
\end{array}\right), \quad \text { if } \quad c<z, \\
& S_{+}(z)=S_{-}(z)\left(\begin{array}{cc}
0 & |z-t|^{\alpha} \\
-|z-t|^{-\alpha} & 0
\end{array}\right), \quad \text { if } \quad z \in \mathcal{S}, \\
& S_{+}(z)=S_{-}(z)\left(\begin{array}{cc}
1 & 0 \\
(z-t)^{-\alpha} e^{-2 n \xi_{2}(z)} e^{2 \pi i \Omega n} & 1
\end{array}\right), \quad \text { if } \quad z \in \tilde{\gamma}_{+} \text {, } \\
& S_{+}(z)=S_{-}(z)\left(\begin{array}{cc}
1 & 0 \\
(z-t)^{-\alpha} e^{-2 n \xi_{2}(z)} e^{-2 \pi i \Omega n} & 1
\end{array}\right), \quad \text { if } \quad z \in \tilde{\gamma}_{-}, \\
& S_{+}(z)=S_{-}(z)\left(\begin{array}{cc}
1 & 0 \\
(z-t)^{-\alpha} e^{-2 n \xi_{1}(z)} & 1
\end{array}\right), \quad \text { if } \quad z \in \gamma_{+} \cup \gamma_{-} .
\end{aligned}
$$

(c) As $z \rightarrow \infty$, we have $S(z)=I+\mathcal{O}\left(z^{-1}\right)$.

(d) As $z$ tends to $t$, we have

$$
\begin{aligned}
& S(z)=\left\{\begin{array}{ll}
\left(\begin{array}{ll}
\mathcal{O}(1) & \mathcal{O}(\log (z-t)) \\
\mathcal{O}(1) & \mathcal{O}(\log (z-t))
\end{array}\right), \quad \text { zoutside the lenses, } \\
\mathcal{O}(\log (z-t)) & \mathcal{O}(\log (z-t)) \\
\mathcal{O}(\log (z-t)) & \mathcal{O}(\log (z-t))
\end{array}\right), \quad z \text { inside the lenses, } \quad \alpha=0, \\
& S(z)=\left\{\begin{array}{ll}
\left(\begin{array}{ll}
\mathcal{O}(1) & \mathcal{O}(1) \\
\mathcal{O}(1) & \mathcal{O}(1)
\end{array}\right), & z \text { outside the lenses, } \\
\mathcal{O}\left((z-t)^{-\alpha}\right) & \mathcal{O}(1) \\
\mathcal{O}\left((z-t)^{-\alpha}\right) & \mathcal{O}(1)
\end{array}\right), \quad z \text { inside the lenses, } \quad \alpha>0, \\
& S(z)=\left(\begin{array}{ll}
\mathcal{O}(1) & \mathcal{O}\left((z-t)^{\alpha}\right) \\
\mathcal{O}(1) & \mathcal{O}\left((z-t)^{\alpha}\right)
\end{array}\right), \quad \text { if } \quad \alpha<0 .
\end{aligned}
$$

As $z$ tends to $a, b$ or $c$, we have $S(z)=\mathcal{O}(1)$.

From (6.5), (6.7), (6.8) and the fact that $\xi_{1}(z)<0$ for $z>c$, the jumps for $S$ on the boundary of the lenses are exponentially close to the identity matrix as $n \rightarrow \infty$ and the $(1,2)$ entries of the jumps on $\mathbb{R} \backslash \mathcal{S}$ are exponentially small as $n \rightarrow \infty$, but these convergences are not uniform 


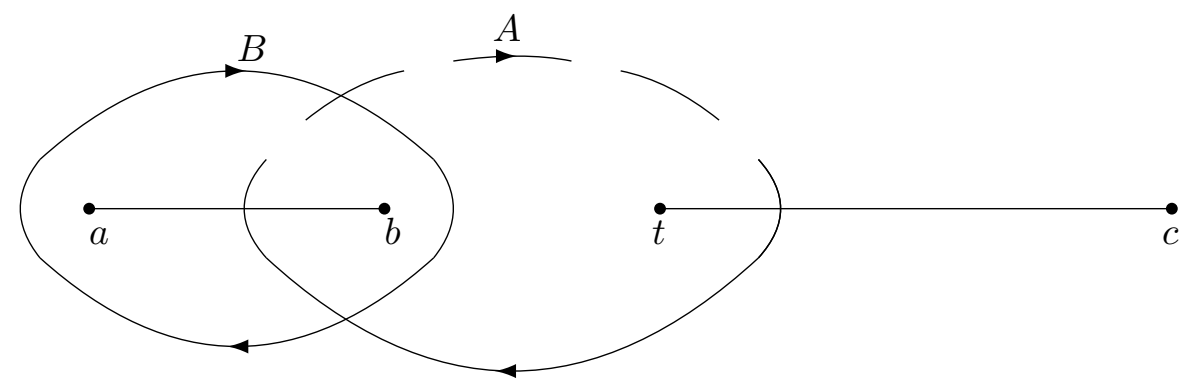

Figure 4. The cycles $A$ and $B$. The solid line of $A$ is in the first sheet and the dashed line is in the second sheet. The cycle $B$ lies on the first sheet.

for $z$ in a neighbourhood of $a, b, t$ and $c$. By ignoring the exponentially small terms in the jumps for $S$, we are left with a simpler RH problem, whose solution $P^{(\infty)}$ will be a good approximation of $S$ away from neighbourhoods of $a, b, t$ and $c$. We construct $P^{(\infty)}$ explicitly in Section 6.2.

\subsection{Global parametrix}

If we ignore the exponentially small terms as $n \rightarrow \infty$ in the jumps for $S$ and a small neighbourhood of $a, b, t$ and $c$, we are left with a simpler RH problem, whose solution $P^{(\infty)}$ is called the global parametrix, and is a good approximation of $S$ away from a neighbourhood of $a, b, t$ and $c$.

\section{RH problem for $\boldsymbol{P}^{(\infty)}$}

(a) $P^{(\infty)}: \mathbb{C} \backslash[a, c] \rightarrow \mathbb{C}^{2 \times 2}$ is analytic.

(b) $P^{(\infty)}$ has the following jumps:

$$
\begin{aligned}
& P_{+}^{(\infty)}(z)=P_{-}^{(\infty)}(z)\left(\begin{array}{cc}
0 & |z-t|^{\alpha} \\
-|z-t|^{-\alpha} & 0
\end{array}\right), \quad \text { if } \quad z \in \mathcal{S}, \\
& P_{+}^{(\infty)}(z)=P_{-}^{(\infty)}(z) e^{2 \pi i \Omega n \sigma_{3}}, \quad \text { if } \quad z \in(b, t) .
\end{aligned}
$$

(c) As $z \rightarrow \infty$, we have $P^{(\infty)}(z)=I+\mathcal{O}\left(z^{-1}\right)$.

(d) As $z$ tends to $\tilde{z} \in\{a, b, c\}$, we have $P^{(\infty)}(z)=\mathcal{O}\left((z-\tilde{z})^{-1 / 4}\right)$.

As $z$ tends to $t$, we have $P^{(\infty)}(z)=\mathcal{O}\left((z-t)^{-1 / 4}\right)(z-t)^{-\frac{\alpha}{2} \sigma_{3}}$.

The construction of $P^{(\infty)}$ has been done in similar situations in [19] for $\alpha=0$ and in [31] for $\alpha \neq 0$. It involves $\theta$-functions and quantities related to a Riemann surface. Let $X$ be the two sheeted Riemann surface of genus one associated to $\sqrt{R(z)}$, with

$$
R(z)=(z-c)(z-t)(z-b)(z-a),
$$

and we let $\sqrt{R(z)} \sim z^{2}$ as $z \rightarrow \infty$ on the first sheet. We also define cycles $A$ and $B$ such that they form a canonical homology basis of $X$. The upper part of the cycle $A$ (the dashed line in Fig. 4) lies on the second sheet, and the lower part lies on the first sheet. The cycle $B$ surrounds $(a, b)$ in the clockwise direction, and lie in the first sheet. The unique $A$-normalized holomorphic one-form $\omega$ on $X$ is given by

$$
\omega=\frac{c_{0} \mathrm{~d} z}{\sqrt{R(z)}}, \quad c_{0}=\left(\int_{A} \frac{1}{\sqrt{R(z)}} \mathrm{d} z\right)^{-1} .
$$


By construction $\int_{A} \omega=1$ and the lattice parameter is given by $\tau=\int_{B} \omega$. A direct calculation shows that

$$
c_{0}=\left(\int_{b}^{t} \frac{2}{\sqrt{|R(x)|}} \mathrm{d} x\right)^{-1} \in \mathbb{R}^{+}, \quad \tau=\int_{a}^{b} \frac{2 i c_{0}}{\sqrt{|R(x)|}} \mathrm{d} x \in i \mathbb{R}^{+} .
$$

The associated $\theta$-function of the third kind $\theta(z)=\theta(z ; \tau)$ is given by

$$
\theta(z)=\sum_{m=-\infty}^{\infty} e^{2 \pi i m z} e^{\pi i m^{2} \tau}
$$

It is an entire function which satisfies

$$
\theta(z+1)=\theta(z), \quad \theta(z+\tau)=e^{-2 \pi i z} e^{-\pi i \tau} \theta(z), \quad \text { for all } \quad z \in \mathbb{C} .
$$

We also need the function

$$
u(z)=\int_{c}^{z} \omega
$$

where the path of integration lies in $\mathbb{C} \backslash[a, c)$. Since $\int_{C} \omega=0$ for any circle $C$ winding around $\mathcal{S}$, $u$ is a single valued function for $z \in \mathbb{C} \backslash[a, c)$. For $z$ on the first sheet, it satisfies

$$
\begin{array}{ll}
u_{+}(z)+u_{-}(z)=0, & z \in(t, c), \\
u_{+}(z)+u_{-}(z)=1, & z \in(a, b), \\
u_{+}(z)-u_{-}(z)=\tau, & z \in(b, t), \\
\lim _{z \rightarrow \infty} u(z)=u_{\infty} \in \mathbb{C} . &
\end{array}
$$

We define $\beta(z)=\sqrt[4]{\frac{z-a}{z-b} \frac{z-t}{z-c}}$, such that $\beta(z) \sim 1$ as $z \rightarrow \infty$ on the first sheet. On this first sheet, it can be verified that the function $\beta(z)+\beta^{-1}(z)$ never vanishes, while $\beta(z)-\beta^{-1}(z)$ vanishes at a single point $z_{\star}$, given by

$$
z_{\star}=\frac{c-t}{c-t+b-a} b+\frac{b-a}{c-t+b-a} t \in(b, t) .
$$

This observation will be useful later, because the solution $P^{(\infty)}$ will involve functions of the form $1 / \theta(u(z) \pm d)$, where $d=-\frac{1}{2}-\frac{\tau}{2}+\int_{c}^{z_{\star}} \omega$. The function $1 / \theta(u(z)+d)$ has no pole on the first sheet, while the function $1 / \theta(u(z)-d)$ has a pole at $z_{\star}$, see [19]. Therefore, the functions $\frac{\beta(z) \pm \beta^{-1}(z)}{\theta(u(z) \pm d)}$ are analytic on the first sheet.

Before stating the solution $P^{(\infty)}$, we follow [31] and introduce a scalar Szegö function $D$ which satisfies

(a) $D: \mathbb{C} \backslash[a, c] \rightarrow \mathbb{C}$ is analytic.

(b) $D$ has the following jumps:

$$
\begin{aligned}
& D_{+}(z) D_{-}(z)=|z-t|^{\alpha}, \quad \text { if } \quad z \in \mathcal{S}, \\
& D_{+}(z)=D_{-}(z) e^{2 \pi i \tilde{\alpha}}, \quad \text { if } \quad z \in(b, t) .
\end{aligned}
$$

(c) As $z \rightarrow \infty$, we have $D(z)=D_{\infty}+\mathcal{O}\left(z^{-1}\right), D_{\infty} \neq 0$.

(d) As $z \rightarrow t, D(z)=(z-t)^{\frac{\alpha}{2}} d_{t}+o\left((z-t)^{\frac{\alpha}{2}}\right), d_{t} \neq 0$.

As $z$ tends to $\tilde{z} \in\{a, b, c\}, D(z)$ is bounded. 
The new parameter $\tilde{\alpha}$ is not arbitrary and is completely determined to ensure the existence of $D$. The solution is given by

$$
D(z)=\exp \left(\sqrt{R(z)}\left[\frac{1}{2 \pi i} \int_{\mathcal{S}} \frac{\alpha \log |x-t|}{\sqrt{R(x)}+} \frac{\mathrm{d} x}{x-z}+\tilde{\alpha} \int_{b}^{t} \frac{1}{\sqrt{R(x)}} \frac{\mathrm{d} x}{x-z}\right]\right),
$$

see [31] for a detailed proof of it, and $\tilde{\alpha}$ is chosen such that $D(z)$ remains bounded as $z \rightarrow \infty$ :

$$
\tilde{\alpha}=-\left(\int_{b}^{t} \frac{1}{\sqrt{R(x)}} \mathrm{d} x\right)^{-1} \frac{1}{2 \pi i} \int_{\mathcal{S}} \frac{\alpha \log |x-t|}{\sqrt{R(x)}} \mathrm{d} x .
$$

From (6.11), by expanding $\frac{1}{x-z}=-\frac{1}{z}\left(1+\frac{x}{z}+\mathcal{O}\left(z^{-2}\right)\right)$ as $z \rightarrow \infty$, we can evaluate $D_{\infty}$. We obtain

$$
D_{\infty}=\exp \left(-\frac{1}{2 \pi i} \int_{\mathcal{S}} \frac{\alpha x \log |x-t|}{\sqrt{R(x)}+} \mathrm{d} x-\tilde{\alpha} \int_{b}^{t} \frac{x}{\sqrt{R(x)}} \mathrm{d} x\right) .
$$

The solution $P^{(\infty)}$ is given by (see [31, Section 4], and [19, Lemma 4.3])

$$
P^{(\infty)}(z)=\frac{D_{\infty}^{\sigma_{3}}}{2}\left(\begin{array}{cc}
\beta(z)+\beta^{-1}(z) \Theta_{11}(z) & i\left(\beta(z)-\beta^{-1}(z)\right) \Theta_{12}(z) \\
-i\left(\beta(z)-\beta^{-1}(z)\right) \Theta_{21}(z) & \beta(z)+\beta^{-1}(z) \Theta_{22}(z)
\end{array}\right) D(z)^{-\sigma_{3}},
$$

where

$$
\begin{array}{ll}
\Theta_{11}(z)=\frac{\theta\left(u_{\infty}+d\right) \theta(u(z)+d-\Omega n-\tilde{\alpha})}{\theta\left(u_{\infty}+d-\Omega n-\tilde{\alpha}\right) \theta(u(z)+d)}, & \Theta_{12}(z)=\frac{\theta\left(u_{\infty}+d\right) \theta(u(z)-d+\Omega n+\tilde{\alpha})}{\theta\left(u_{\infty}+d-\Omega n-\tilde{\alpha}\right) \theta(u(z)-d)} \\
\Theta_{21}(z)=\frac{\theta\left(u_{\infty}+d\right) \theta(u(z)-d-\Omega n-\tilde{\alpha})}{\theta\left(u_{\infty}+d+\Omega n+\tilde{\alpha}\right) \theta(u(z)-d)}, & \Theta_{22}(z)=\frac{\theta\left(u_{\infty}+d\right) \theta(u(z)+d+\Omega n+\tilde{\alpha})}{\theta\left(u_{\infty}+d+\Omega n+\tilde{\alpha}\right) \theta(u(z)+d)}
\end{array}
$$

\subsection{Local parametrices}

By the assumption made at the beginning of Section 6, i.e., that $(t, \lambda)$ is in a compact subset of $\mathcal{R}$ as $n \rightarrow \infty$, by Proposition 4.3, there exists $\delta>0$ independent of $n$ such that

$$
\delta<\min \{b-a, t-b, c-t\} .
$$

We consider some disks $D_{a}, D_{b}, D_{t}, D_{c}$ around $a, b, t$ and $c$ respectively, such that the radii are smaller than $\delta / 3$ but fixed. Inside these disks, we require the local parametrices $P$ to have the same jumps as $S$, and the same behaviour near $a, b, t$ and $c$. Furthermore, uniformly for $z$ on the boundary of these disks, $P$ satisfies the matching condition

$$
P(z)=\left(I+\mathcal{O}\left(n^{-1}\right)\right) P^{(\infty)}(z), \quad \text { as } n \rightarrow \infty .
$$

The solution $P$ of the local parametrices around $a, b$ and $c$ is constructed in terms of the model Airy $\mathrm{RH}$ problem, and the local parametrix $P$ around $t$ in terms of the modified Bessel model $\mathrm{RH}$ problem. As these constructions do not present any additional technicalities other than the ones in Section 5, and as we will not use them explicitly later, we have decided not to include them in the present article.

\subsection{Small norm $\mathrm{RH}$ problem}

The final transformation is given by

$$
R(z)= \begin{cases}S(z) P^{(\infty)}(z)^{-1}, & z \in \mathbb{C} \backslash \bar{D} \\ S(z) P(z)^{-1}, & z \in D\end{cases}
$$

where $D=D_{a} \cup D_{b} \cup D_{t} \cup D_{c}$. Since $P$ has exactly the same jumps as $S$ inside $D$, and the same behaviour near $a, b, t$ and $c$, we show in a similar way as done at the beginning of Section 5.6 that $R$ is analytic inside these disks and it satisfies the following $\mathrm{RH}$ problem. 


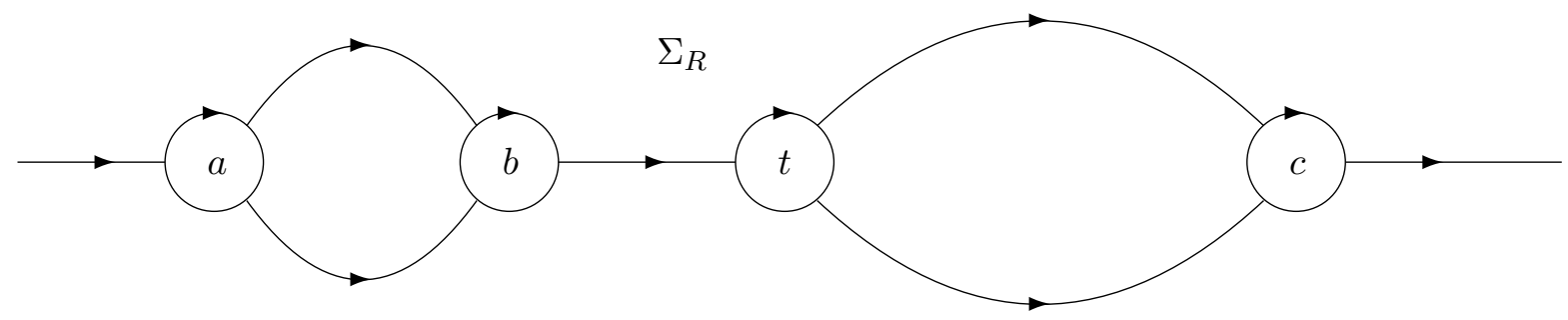

Figure 5. Jump contour for $R$. The orientation on the circles is clockwise.

\section{RH problem for $R$}

(a) $R: \mathbb{C} \backslash \Sigma_{R} \rightarrow \mathbb{C}^{2 \times 2}$ is analytic, with the contour $\Sigma_{R}$ shown in Fig. 5 .

(b) The jumps $J_{R}(z):=R_{-}^{-1}(z) R_{+}(z)$ satisfy the following large $n$ asymptotics for $z \in \Sigma_{R}$ :

$$
\begin{aligned}
& J_{R}(z)=I+\mathcal{O}\left(e^{-c n}\right), \quad \text { uniformly for } \quad z \in\left(\gamma_{+} \cup \gamma_{-} \cup \tilde{\gamma}_{+} \cup \tilde{\gamma}_{-} \cup \mathbb{R}\right) \backslash(\mathcal{S} \cup \bar{D}), \\
& J_{R}(z)=I+\mathcal{O}\left(n^{-1}\right), \quad \text { uniformly for } \quad z \in \partial D,
\end{aligned}
$$

where $c>0$ is a constant.

(c) As $z \rightarrow \infty$, we have $R(z)=I+\mathcal{O}\left(z^{-1}\right)$.

Again, from standard theory for small-norm RH problems [18], $R$ exists for sufficiently large $n$ and we have

$$
R(z)=I+\mathcal{O}\left(n^{-1}\right), \quad R^{\prime}(z)=\mathcal{O}\left(n^{-1}\right),
$$

uniformly for $z \in \mathbb{C} \backslash \Sigma_{R}$, and uniformly for $(t, \lambda)$ in a compact subset of $\mathcal{R}$.

\section{Asymptotics for the Hankel determinant $H_{n}(v, s, \alpha)$}

\subsection{Proof of Theorem 2.1}

In this section, we use the RH analysis done in Section 5 with $\lambda=+\infty$ (i.e., $s=0$ ) and the differential identity

$$
\partial_{t} \log H_{n}(\sqrt{2 n} t, 0, \alpha)=4 n U_{1,11},
$$

which was obtained in (4.5). Inverting the transformations $R \mapsto S \mapsto T \mapsto U$, we obtain for $z$ outside the disks and outside the lenses that

$$
U(z)=e^{-\frac{n \ell}{2} \sigma_{3}} R(z) P^{(\infty)}(z) e^{n g(z) \sigma_{3}} e^{\frac{n \ell}{2} \sigma_{3}} .
$$

In particular, the $(1,1)$ entry in the above expression is given by

$$
U_{11}(z)=e^{n g(z)} P_{11}^{(\infty)}(z)\left(R_{11}(z)+R_{12}(z) \frac{P_{21}^{(\infty)}(z)}{P_{11}^{(\infty)}(z)}\right) .
$$

Thus, by equations (4.28) and (5.12), we have

$$
U_{1,11}=-n \int_{\mathcal{S}} x \rho(x) \mathrm{d} x-\frac{\alpha(\bar{c}-t)}{4}+\frac{R_{1,11}^{(1)}}{n}+\mathcal{O}\left(n^{-2}\right), \quad \text { as } \quad n \rightarrow \infty,
$$


where the $\mathcal{O}\left(n^{-2}\right)$ is uniform for $t$ in compact subsets of $(-1, \infty)$, and $R_{1,11}^{(1)}$ is the coefficient of the $z^{-1}$ term in the large $z$ expansion of $R_{11}^{(1)}(z)$. From (5.27), it is given by

$$
R_{1,11}^{(1)}=\sqrt{\bar{c}-t} \frac{4 \alpha^{2}-1}{32 k_{1}}+\frac{-8 \alpha^{2}+2(\bar{c}-t) k_{3}+3}{64 \sqrt{\bar{c}-t} k_{2}} .
$$

On the other hand, by using (4.9) we can calculate explicitly the first moment of $\rho$, we have

$$
\int_{t}^{\bar{c}} x \rho(x) \mathrm{d} x=\frac{2}{27}\left(\sqrt{3+t^{2}}-t\right)\left(3+5 t^{2}+4 t \sqrt{3+t^{2}}\right) .
$$

Thus, we can rewrite the differential identity (7.1) more explicitly:

$$
\partial_{t} \log H_{n}(\sqrt{2 n} t, 0, \alpha)=u_{1}(t) n^{2}+u_{2}(t, \alpha) n+u_{3}(t, \alpha)+\mathcal{O}\left(n^{-1}\right),
$$

where

$$
\begin{aligned}
& u_{1}(t)=-\frac{8}{27}\left(\sqrt{3+t^{2}}-t\right)\left(3+5 t^{2}+4 t \sqrt{3+t^{2}}\right), \\
& u_{2}(t, \alpha)=-\frac{2 \alpha}{3}\left(\sqrt{3+t^{2}}-t\right), \\
& u_{3}(t, \alpha)=\frac{\left(\sqrt{3+t^{2}}-t\right)\left(t+\sqrt{3+t^{2}}\left(6 \alpha^{2}-1\right)\right)}{12\left(3+t^{2}\right)\left(2 t+\sqrt{3+t^{2}}\right)} .
\end{aligned}
$$

Note that for $t>-1$ we have $\int_{0}^{t} u_{1}(x) \mathrm{d} x=C_{1}(t), \int_{0}^{t} u_{2}(x, \alpha) \mathrm{d} x=C_{2}(t, \alpha)$ and $\int_{0}^{t} u_{3}(x, \alpha) \mathrm{d} x=$ $C_{3}(t, \alpha)$, where $C_{1}(t), C_{2}(t, \alpha)$ and $C_{3}(t, \alpha)$ have been defined in (2.2), (2.3) and (2.4) respectively. Since the $\mathcal{O}\left(n^{-1}\right)$ term in $(7.2)$ is uniform for $t$ in compact subsets of $(-1, \infty)$ and for $\lambda \geq \lambda_{c}(t)$, this gives the result.

\subsection{Proof of Theorem 2.3(1)}

In this section, we again use the $\mathrm{RH}$ analysis done in Section 5. In order to use the differential identity (4.6), we need to obtain large $n$ asymptotics for $U$ uniformly on $(-\infty, t)$. This can be achieved by inverting the transformations $R \mapsto S \mapsto T \mapsto U$ in different regions. For $z \in D_{\bar{b}} \backslash \mathbb{R}$, we have

$$
U(z)=e^{-\frac{n \ell}{2} \sigma_{3}} R(z) P^{(\infty)}(z)\left(\begin{array}{cc}
1 & l(z) \\
0 & 1
\end{array}\right) e^{n g(z) \sigma_{3}} e^{\frac{n \ell}{2} \sigma_{3}} .
$$

For $z$ outside the lenses and outside the disks, $z \notin \mathbb{R}$, the expression for $U$ in terms of $R$ is

$$
U(z)=e^{-\frac{n \ell}{2} \sigma_{3}} R(z) P^{(\infty)}(z) e^{n g(z) \sigma_{3}} e^{\frac{n \ell}{2} \sigma_{3}} .
$$

Thus, from (7.3) and (7.4), for all $z$ such that $z \notin \mathbb{R}$ and $\Re z<t, z \notin D_{t}$, we obtain

$$
\left[U^{-1}(z) U^{\prime}(z)\right]_{21}=e^{n \ell} e^{2 n g(z)}\left[P^{(\infty)}(z)^{-1} R(z)^{-1} R^{\prime}(z) P^{(\infty)}(z)+P^{(\infty)}(z)^{-1} P^{(\infty)}(z)^{\prime}\right]_{21} .
$$

From (5.11), as $n \rightarrow \infty$ we have the following bounds for the global parametrix:

$$
P^{(\infty)}(z)=\mathcal{O}(1), \quad P^{(\infty)}(z)^{\prime}=\mathcal{O}(1), \quad \text { uniformly for } z \text { outside the disks. }
$$

Furthermore, by using the estimate for $R$ given by (5.26), and by taking the limit $z \rightarrow x \in \mathbb{R}$ in equation (7.5) (the limits from the upper and lower half plane are the same, see Remark 4.2), we obtain

$$
\frac{\tilde{w}(x)}{2 \pi i}\left[U^{-1}(x) U^{\prime}(x)\right]_{21}=|x-t|^{\alpha} e^{n\left(g_{+}(x)+g_{-}(x)+\ell-V(x)\right)} \mathcal{O}(1), \quad \text { as } \quad n \rightarrow \infty,
$$

where the $\mathcal{O}(1)$ is uniform for $x \in(-\infty, t), x \notin D_{t}$. It is more complicated to obtain similar

asymptotics for $\frac{\tilde{w}(x)}{2 \pi i}\left[U^{-1}(x) U^{\prime}(x)\right]_{21}$, uniformly for $x \in(-\infty, t) \cap D_{t}$. We will need the following lemma. 
Lemma 7.1. As $n \rightarrow \infty$, we have

$$
\begin{aligned}
& U_{+}(x)\left(\begin{array}{l}
1 \\
0
\end{array}\right)=e^{\frac{n \ell}{2}} e^{n g_{+}(x)} e^{-\frac{n \ell}{2} \sigma_{3}}\left(\begin{array}{l}
\mathcal{O}\left(n^{\frac{1}{2}+\max (\alpha, 0)}\right) \\
\mathcal{O}\left(n^{\frac{1}{2}+\max (\alpha, 0)}\right)
\end{array}\right), \\
& U_{+}^{\prime}(x)\left(\begin{array}{l}
1 \\
0
\end{array}\right)=e^{\frac{n \ell}{2}} e^{n g_{+}(x)} e^{-\frac{n \ell}{2} \sigma_{3}}\left(\begin{array}{l}
\mathcal{O}\left(n^{\frac{5}{2}+\max (\alpha, 0)}\right) \\
\mathcal{O}\left(n^{\frac{5}{2}+\max (\alpha, 0)}\right)
\end{array}\right),
\end{aligned}
$$

uniformly for $x \in(-\infty, t) \cap D_{t}$.

Proof. For $z \in D_{t}, z$ outside the lenses, we have

$$
U(z)\left(\begin{array}{l}
1 \\
0
\end{array}\right)=e^{\frac{n \ell}{2}} e^{n g(z)} e^{-\frac{n \ell}{2} \sigma_{3}} R(z) P(z)\left(\begin{array}{l}
1 \\
0
\end{array}\right) .
$$

If furthermore, $\Im z>0$, by (5.19) and (B.4) we have

$$
P(z)\left(\begin{array}{l}
1 \\
0
\end{array}\right)=e^{\frac{\pi i \alpha}{2}}(z-t)^{-\frac{\alpha}{2}} e^{-n \xi(z)} E(z)\left(\begin{array}{c}
I_{\alpha}(2 n \sqrt{-f(z)}) \\
-2 \pi i n \sqrt{-f(z)} I_{\alpha}^{\prime}(2 n \sqrt{-f(z)})
\end{array}\right) .
$$

Let $x \in(-\infty, t) \cap D_{t}$. Note that $\tilde{\xi}(x)>0(\tilde{\xi}$ is defined in $(5.16))$ and thus $\sqrt{-f(x)}+=\frac{1}{2} \tilde{\xi}(x)$. Inserting (7.10) into (7.9), we can take the limit $z \rightarrow x$, this gives

$$
\begin{aligned}
U_{+}(x)\left(\begin{array}{l}
1 \\
0
\end{array}\right)= & (-1)^{n} e^{\frac{n \ell}{2}} e^{n g_{+}(x)} e^{-n \tilde{\xi}(x)}(t-x)^{-\frac{\alpha}{2}} e^{-\frac{n \ell}{2} \sigma_{3}} \\
& \times R(x) E(x)\left(\begin{array}{c}
I_{\alpha}(n \tilde{\xi}(x)) \\
-\pi i n \tilde{\xi}(x) I_{\alpha}^{\prime}(n \tilde{\xi}(x))
\end{array}\right) .
\end{aligned}
$$

Since $E$ is analytic in $D_{t}$, one has from (5.21) that as $n \rightarrow \infty$

$$
E(z)=\mathcal{O}(1) n^{\frac{\sigma_{3}}{2}}, \quad E^{\prime}(z)=\mathcal{O}(1) n^{\frac{\sigma_{3}}{2}}, \quad \text { uniformly for } z \in D_{t} .
$$

To obtain a uniform bound from (7.11), we distinguish three cases. Let $M>0$ be an arbitrary large but fixed constant and let $m>0$ be an arbitrary small but fixed constant.

Case (a): $n \tilde{\xi}(x) \geq M$ as $n \rightarrow \infty$. In this case we need large $\zeta$ asymptotics for $I_{\alpha}(\zeta)$ and $I_{\alpha}^{\prime}(\zeta)$. From (B.2), we have

$$
I_{\alpha}(\zeta)=\frac{e^{\zeta}}{\sqrt{2 \pi \zeta}}\left(1+\mathcal{O}\left(\zeta^{-1}\right)\right), \quad I_{\alpha}^{\prime}(\zeta)=\frac{e^{\zeta}}{\sqrt{2 \pi \zeta}}\left(1+\mathcal{O}\left(\zeta^{-1}\right)\right), \quad \text { as } \quad \zeta \rightarrow \infty
$$

If we insert (7.13) into (7.11), the result follows for Case (a) from (5.26), (7.12) and from the fact that $(t-x)^{-\frac{\alpha}{2}}=\mathcal{O}\left(n^{\max (\alpha, 0)}\right)$.

Case (b): $m \leq n \tilde{\xi}(x) \leq M$ as $n \rightarrow \infty$. In this case we have $I_{\alpha}(n \tilde{\xi}(x))=\mathcal{O}(1), n \tilde{\xi}(x) I_{\alpha}^{\prime}(n \tilde{\xi}(x))$ $=\mathcal{O}(1), e^{-n \tilde{\xi}(x)}=\mathcal{O}(1),(t-x)^{-\frac{\alpha}{2}}=\mathcal{O}\left(n^{\alpha}\right)$. Again from (5.26) and (7.12), we obtain

$$
U_{+}(x)\left(\begin{array}{l}
1 \\
0
\end{array}\right)=e^{\frac{n \ell}{2}} e^{n g_{+}(x)} e^{-\frac{n \ell}{2} \sigma_{3}}\left(\begin{array}{c}
\mathcal{O}\left(n^{\frac{1}{2}+\alpha}\right) \\
\mathcal{O}\left(n^{-\frac{1}{2}+\alpha}\right)
\end{array}\right),
$$

which is even slightly better than (7.7).

Case (c): $n \tilde{\xi}(x) \leq m$ as $n \rightarrow \infty$. From [36, formula (10.25.2)], we have

$$
I_{\alpha}(\zeta)=\left(\frac{\zeta}{2}\right)^{\alpha}\left(\frac{1}{\Gamma(1+\alpha)}+\mathcal{O}\left(\zeta^{2}\right)\right)
$$




$$
I_{\alpha}^{\prime}(\zeta)=\left(\frac{\zeta}{2}\right)^{\alpha-1}\left(\frac{\alpha}{\Gamma(1+\alpha)}+\mathcal{O}\left(\zeta^{2}\right)\right), \quad \text { as } \quad \zeta \rightarrow 0
$$

From the above expansion, we have for Case (c) that

$$
\frac{I_{\alpha}(n \tilde{\xi}(x))}{(t-x)^{\frac{\alpha}{2}}}=\mathcal{O}\left(n^{\alpha}\right), \quad \frac{n \tilde{\xi}(x) I_{\alpha}^{\prime}(n \tilde{\xi}(x))}{(t-x)^{\frac{\alpha}{2}}}=\mathcal{O}\left(n^{\alpha}\right)
$$

and $e^{-n \tilde{\xi}(x)}=\mathcal{O}(1)$. Thus, from (5.26) and (7.12) we obtain again (7.14), which finishes the proof of (7.7). We now turn to the proof of (7.8). From (7.11), we have $U_{+}^{\prime}(x)\left(\begin{array}{l}1 \\ 0\end{array}\right)=\widetilde{U}_{1}(x)+\widetilde{U}_{2}(x)$, where

$$
\begin{aligned}
& \widetilde{U}_{1}(x)= n\left(g_{+}^{\prime}(x)-\tilde{\xi}^{\prime}(x)\right) U_{+}(x)\left(\begin{array}{l}
1 \\
0
\end{array}\right)+(-1)^{n} e^{\frac{n \ell}{2}} e^{n g_{+}(x)} e^{-n \tilde{\xi}(x)}(t-x)^{-\frac{\alpha}{2}} e^{-\frac{n \ell}{2} \sigma_{3}} \\
& \times\left(R^{\prime}(x) E(x)+R(x) E^{\prime}(x)\right)\left(\begin{array}{c}
I_{\alpha}(n \tilde{\xi}(x)) \\
-\pi i n \tilde{\xi}(x) I_{\alpha}^{\prime}(n \tilde{\xi}(x))
\end{array}\right) \\
& \widetilde{U}_{2}(x)=(-1)^{n} e^{\frac{n \ell}{2}} e^{n g_{+}(x)} e^{-n \tilde{\xi}(x)} e^{-\frac{n \ell}{2} \sigma_{3}} R(x) E(x)\left(\begin{array}{c}
\left(\frac{I_{\alpha}(n \tilde{\xi}(x))}{(t-x)^{\frac{\alpha}{2}}}\right)^{\prime} \\
\left(\frac{-\pi i n \tilde{\xi}(x) I_{\alpha}^{\prime}(n \tilde{\xi}(x))}{(t-x)^{\frac{\alpha}{2}}}\right)^{\prime}
\end{array}\right) .
\end{aligned}
$$

The analysis of $\widetilde{U}_{1}(x)$ and $\widetilde{U}_{2}(x)$ can be done very similarly to the first part of the proof and we do not provide here all the details. From (5.4), one has $g_{+}^{\prime}(x)-\tilde{\xi}^{\prime}(x)=2 x$ and thus by (5.26), (7.7) and (7.12),

$$
\widetilde{U}_{1}(x)=e^{\frac{n \ell}{2}} e^{n g_{+}(x)} e^{-\frac{n \ell}{2} \sigma_{3}}\left(\begin{array}{l}
\mathcal{O}\left(n^{\frac{3}{2}+\max (\alpha, 0)}\right) \\
\mathcal{O}\left(n^{\frac{3}{2}+\max (\alpha, 0)}\right)
\end{array}\right) .
$$

Again, by splitting the analysis into the same three cases as in the first part of the proof, we obtain the estimates

$$
\begin{aligned}
& \left(\frac{I_{\alpha}(n \tilde{\xi}(x))}{(t-x)^{\frac{\alpha}{2}}}\right)^{\prime}=\mathcal{O}\left(n^{2}\left(\frac{I_{\alpha}(n \tilde{\xi}(x))}{(t-x)^{\frac{\alpha}{2}}}\right)\right), \\
& \left(\frac{-i \pi n \tilde{\xi}(x) I_{\alpha}^{\prime}(n \xi(x))}{(t-x)^{\frac{\alpha}{2}}}\right)^{\prime}=\mathcal{O}\left(n^{2}\left(\frac{-i \pi n \tilde{\xi}(x) I_{\alpha}^{\prime}(n \xi(x))}{(t-x)^{\frac{\alpha}{2}}}\right)\right),
\end{aligned}
$$

which yields $\widetilde{U}_{2}(x)=e^{\frac{n \ell}{2}} e^{n g_{+}(x)} e^{-\frac{n \ell}{2} \sigma_{3}}\left(\begin{array}{l}\mathcal{O}\left(n^{\frac{5}{2}+\max (\alpha, 0)}\right) \\ \mathcal{O}\left(n^{\frac{5}{2}+\max (\alpha, 0)}\right)\end{array}\right)$ and finishes the proof.

Note that $g_{+}(x)+g_{-}(x)-2 x^{2}+\ell$ is continuous on $\mathbb{R}$ and equal to 0 at $x=t$ by (4.7). Thus, from (4.24) and (4.8) and the fact that $V(x)$ has a jump discontinuity at $x=t$, we have

$$
\lim _{\substack{x \rightarrow t \\ x<t}} g_{+}(x)+g_{-}(x)-V(x)+\ell=-\lambda<-\lambda_{c}<0 .
$$

Therefore, by using first Lemma 7.1 and then (7.15), there exists $c \in\left(0, \lambda_{c}\right)$ such that

$$
\begin{aligned}
\frac{\tilde{w}(x)}{2 \pi i}\left[U^{-1}(x) U^{\prime}(x)\right]_{21} & =|x-t|^{\alpha} e^{n\left(g_{+}(x)+g_{-}(x)-V(x)+\ell\right)} \mathcal{O}\left(n^{3+2 \max (\alpha, 0)}\right) \\
& =|x-t|^{\alpha} \mathcal{O}\left(e^{-(\lambda-c) n}\right),
\end{aligned}
$$


as $n \rightarrow \infty$ uniformly for $x \in D_{t} \cap(-\infty, t)$. Now, we will split the integral of the differential identity (4.6) into two parts:

$$
\begin{aligned}
& s \partial_{s} \log H_{n}(\sqrt{2 n} t, s)=I_{1}(s)+I_{2}(s), \\
& I_{1}(s)=\int_{(-\infty, t) \backslash D_{t}} \frac{\widetilde{w}(x)}{2 \pi i}\left[U^{-1}(x) U^{\prime}(x)\right]_{21} \mathrm{~d} x, \\
& I_{2}(s)=\int_{(-\infty, t) \cap D_{t}} \frac{\widetilde{w}(x)}{2 \pi i}\left[U^{-1}(x) U^{\prime}(x)\right]_{21} \mathrm{~d} x .
\end{aligned}
$$

The first integral can be evaluated using (7.6). By (5.1), (5.2), (5.4) and (5.7) (see also the comment just after), we have $g_{+}(\bar{b})+g_{-}(\bar{b})+\ell-V(\bar{b})=-\left(\lambda-\lambda_{c}\right)$ and

$$
\left.\left(g_{+}(x)+g_{-}(x)+\ell-V(x)\right)^{\prime}\right|_{x=\bar{b}}=0, \quad\left(g_{+}(x)+g_{-}(x)+\ell-\left.V(x)^{\prime \prime}\right|_{x=\bar{b}}<0 .\right.
$$

Therefore, we obtain

$$
\left|I_{1}\left(s=e^{-\lambda n}\right)\right|=\mathcal{O}\left(n^{-1 / 2} e^{-n\left(\lambda-\lambda_{c}\right)}\right), \quad \text { as } \quad n \rightarrow \infty .
$$

On the other hand, from (7.16), it immediately follows that

$$
\left|I_{2}\left(s=e^{-\lambda n}\right)\right|=\mathcal{O}\left(e^{-(\lambda-c) n}\right), \quad \text { as } \quad n \rightarrow \infty,
$$

where $c \in\left(0, \lambda_{c}\right)$. Therefore, the differential identity becomes

$$
\left.\partial_{s} \log H_{n}(v, s, \alpha)\right|_{s=e^{-\lambda n}}=\mathcal{O}\left(n^{-1 / 2} e^{n \lambda_{c}}\right), \quad \text { as } \quad n \rightarrow \infty,
$$

where in the above expression the $\mathcal{O}$ term is uniform for $t$ in a compact subset of $(-1, \infty)$ and for $\lambda \geq \lambda_{c}(t)$. Thus, we can integrate it from $s=0$ to $s=e^{-\lambda n}$, and it gives

$$
\log H_{n}\left(\sqrt{2 n} t, e^{-\lambda n}, \alpha\right)=\log H_{n}(\sqrt{2 n} t, 0, \alpha)+\mathcal{O}\left(n^{-1 / 2} e^{-n\left(\lambda-\lambda_{c}(t)\right)}\right), \quad \text { as } \quad n \rightarrow \infty,
$$

which is the claim (2.7).

\subsection{Proof of Theorem 2.3(2)}

In this section we use the RH analysis done in Section 6.

Proposition 7.2. Let $\mathcal{W} \subset \mathbb{R}$ be an arbitrary small but fixed neighbourhood of the four points $\{a, b, t, c\}$. We have as $n \rightarrow \infty$

$$
\frac{\tilde{w}(x)}{2 \pi i}\left[U^{-1}(x) U^{\prime}(x)\right]_{21}-n \rho(x) \chi_{\mathcal{S}}(x)=e^{n\left(g_{+}(x)+g_{-}(x)+\ell-V(x)\right)} \mathcal{O}(1),
$$

uniformly for $x \in \mathbb{R} \backslash \mathcal{W}$.

Proof. We can assume without loss of generality that the disks of the local parametrices are sufficiently small such that $D \subset \mathcal{W}$. Let $z$ be outside the lenses and outside the disks. In this region, by inverting the transformations $R \mapsto S \mapsto T \mapsto U$, we have

$$
U(z)=e^{-\frac{n \ell}{2} \sigma_{3}} R(z) P^{(\infty)}(z) e^{n g(z) \sigma_{3}} e^{\frac{n \ell}{2} \sigma_{3}} .
$$

Since the dependence in $n$ of the global parametrix (6.12) appears only in the form $n \Omega \in \mathbb{R}$, and as an argument of the $\theta$-function, by the periodicity property (6.10), as $n \rightarrow \infty$ we have

$$
P^{(\infty)}(z)=\mathcal{O}(1), \quad P^{(\infty)}(z)^{\prime}=\mathcal{O}(1), \quad \text { uniformly for } z \text { outside the disks. }
$$


Therefore, using also the large $n$ asymptotics for $R$ (6.13), we have

$$
\left[U(z)^{-1} U^{\prime}(z)\right]_{21}=e^{n \ell} e^{2 n g(z)} \mathcal{O}(1), \quad \text { as } n \rightarrow \infty,
$$

uniformly for $z$ outside the lenses and outside the disks. For $x \in \mathbb{R} \backslash(\mathcal{S} \cup \mathcal{W})$, we can take the limit $z \rightarrow x$ in (7.17). As $n \rightarrow \infty$, we have

$$
\frac{\tilde{w}(x)}{2 \pi i}\left[U^{-1}(x) U^{\prime}(x)\right]_{21}=|x-t|^{\alpha} e^{n\left(g_{+}(x)+g_{-}(x)+\ell-V(x)\right)} \mathcal{O}(1),
$$

uniformly for $x \in \mathbb{R} \backslash(\mathcal{S} \cup \mathcal{W})$. Now, we consider the case when $z$ is still outside the disks but inside $\mathcal{I}_{1}$, see (6.9) and Fig. 3. Inverting the transformations in this region, we get

$$
U(z)=e^{-\frac{n \ell}{2} \sigma_{3}} R(z) P^{(\infty)}(z)\left(\begin{array}{cc}
1 & 0 \\
(z-t)^{-\alpha} e^{-2 n \xi_{1}(z)} & 1
\end{array}\right) e^{n g(z) \sigma_{3}} e^{\frac{n \ell}{2} \sigma_{3}} .
$$

Since $P^{(\infty)}(z)=\mathcal{O}(1)$ as $n \rightarrow \infty$ uniformly for $z$ in this region, we have

$$
\left[U^{-1}(z) U^{\prime}(z)\right]_{21}=(z-t)^{-\alpha} e^{n(2 g(z)+\ell)}\left(-2 n \xi_{1}^{\prime}(z) e^{-2 n \xi_{1}(z)}+\mathcal{O}(1)\right), \quad \text { as } \quad n \rightarrow \infty,
$$

where we have also used (6.7) and $\Re \xi_{1,+}(x)=0$ for $x \in(t, c)$. Note that from (6.1), we have $\xi_{1,+}^{\prime}(x)=-\pi i \rho(x)$ for $x \in(t, c)$. Thus, if we let $z \rightarrow x \in(t, c) \backslash \mathcal{W}$ in (7.18), from (4.7) and (6.3), we have

$$
\frac{\tilde{w}(x)}{2 \pi i}\left[U^{-1}(x) U^{\prime}(x)\right]_{21}=n \rho(x)\left(1+\mathcal{O}\left(n^{-1}\right)\right), \quad \text { as } \quad n \rightarrow \infty,
$$

where the $\mathcal{O}$ term in the above expression is uniform for $x \in(t, c) \backslash \mathcal{W}$. For $x \in(a, b) \backslash \mathcal{W}$, we can invert the transformations for $z \in \widetilde{\mathcal{I}}_{1}$ and then take the limit $z \rightarrow x$. The computations are similar and we obtain the same asymptotics as (7.19).

By (4.1) and (4.4), note that (3.10) can be rewritten as $\int_{\mathbb{R}} \frac{\tilde{w}(x)}{2 \pi i}\left[U^{-1}(x) U^{\prime}(x)\right]_{21} \mathrm{~d} x=n$. Thus, a consequence of Proposition 7.2 (by taking $\mathcal{W}$ arbitrarily small) and (4.6) is that for fixed $t \in(-1,1)$ and fixed $\lambda \in\left(0, \lambda_{c}(t)\right)$, we have

$$
\left.\lim _{n \rightarrow \infty} \frac{s}{n} \partial_{s} \log H_{n}(\sqrt{2 n} t, s, \alpha)\right|_{s=e^{-\lambda n}}=\lim _{n \rightarrow \infty} \int_{-\infty}^{t} \frac{\widetilde{w}(x)}{2 \pi i n}\left[U^{-1}(x) U^{\prime}(x)\right]_{21} \mathrm{~d} x=\Omega(t, \lambda) .
$$

A simple change of variables shows that

$$
\left.\frac{s}{n} \partial_{s} \log H_{n}(\sqrt{2 n} t, s, \alpha)\right|_{s=e^{-\lambda n}}=-\frac{1}{n^{2}} \partial_{\lambda} \log H_{n}\left(\sqrt{2 n} t, e^{-\lambda n}, \alpha\right) .
$$

By (7.20), for every $(t, \lambda)$ such that $t \in(-1,1)$ and $\lambda \in\left(0, \lambda_{c}(t)\right)$, the right-hand side of the above expression converges to $\Omega(t, \lambda)$ as $n \rightarrow \infty$. Also, by (3.8) and (3.9), we have

$$
\frac{s}{n} \partial_{s} \log H_{n}(v, s, \alpha)=\frac{\mathcal{E}_{n}(v, s, \alpha)}{n} \leq 1 .
$$

Since the constant function 1 is integrable on any bounded interval, we can apply Lebesgue's dominated convergence theorem, and we have

$$
\lim _{n \rightarrow \infty} \frac{-1}{n^{2}} \int_{0}^{\lambda} \partial_{\tilde{\lambda}} \log H_{n}\left(\sqrt{2 n} t, e^{-\tilde{\lambda} n}, \alpha\right) \mathrm{d} \tilde{\lambda}=\int_{0}^{\lambda} \Omega(t, \tilde{\lambda}) \mathrm{d} \tilde{\lambda}
$$

which finishes the proof.

Remark 7.3. As mentioned in Remark 4.5, we have indeed only used pointwise convergence for $\lambda \in\left(0, \lambda_{c}(t)\right)$ of the quantity $\left.\frac{s}{n} \partial_{s} \log H_{n}(\sqrt{2 n} t, s, \alpha)\right|_{s=e^{-\lambda n}}$ to $\Omega(t, \lambda)$ as $n \rightarrow \infty$ and Lebesgue's theorem. The technical RH analysis as $\lambda \rightarrow 0$ or $\lambda \rightarrow \lambda_{c}(t)$ was thus not needed. 


\subsection{Direct proof of formula (2.11)}

In this section we suppose that $t \in(-1,1)$ and $\lambda \in\left(0, \lambda_{c}(t)\right)$, but as we will have to integrate in $\lambda$ over the interval $\left[0, \lambda_{c}(t)\right]$, some quantities need also to be defined for $\lambda=0$ and for $\lambda=\lambda_{c}(t)$. The quantities $\rho(x ; t, \lambda)$ and $\ell(t, \lambda)$ refer to (4.11) and (4.15) if $\lambda \in\left(0, \lambda_{c}(t)\right)$, to (4.9) and (4.10) if $\lambda=\lambda_{c}(t)$, and to (4.16) if $\lambda=0$. Also, $\Omega(t, \lambda)$ is given by $(6.6)$ for $\lambda \in\left(0, \lambda_{c}(t)\right)$, and we define by continuity $\Omega\left(t, \lambda_{c}(t)\right)=0$.

Lemma 7.4. For $t \in(-1,1)$ and $\lambda \in\left(0, \lambda_{c}(t)\right)$, there holds a relation between $\Omega(t, \lambda)$, the density $\rho(x ; t, \lambda)$ given by $(4.11)$, and the Euler-Lagrange constant $\ell(t, \lambda)$ given by $(4.15)$ :

$$
\Omega(t, \lambda)=\partial_{\lambda} \ell(t, \lambda)+\partial_{\lambda} \int_{\mathcal{S}} 2 x^{2} \rho(x ; t, \lambda) \mathrm{d} x+\lambda \partial_{\lambda} \Omega(t, \lambda) .
$$

Proof. Consider the function

$$
H(x ; t, \lambda)=-2 \int_{\mathcal{S}} \log |x-y| \rho(y ; t, \lambda) \mathrm{d} y .
$$

By the Euler-Lagrange equality (4.7), we have

$$
\begin{aligned}
& H(x ; t, \lambda)=\ell(t, \lambda)-2 x^{2}, \quad x \in(t, c), \\
& H(x ; t, \lambda)=\ell(t, \lambda)-2 x^{2}-\lambda, \quad x \in(a, b) .
\end{aligned}
$$

Thus, by integrating it with respect to $\rho(x ; t, \lambda) \mathrm{d} x$, we obtain

$$
\int_{\mathcal{S}} H(x ; t, \lambda) \rho(x ; t, \lambda) \mathrm{d} x=\ell(t, \lambda)-\lambda \Omega(t, \lambda)-\int_{\mathcal{S}} 2 x^{2} \rho(x ; t, \lambda) \mathrm{d} x .
$$

We will evaluate $\partial_{\lambda} \int_{\mathcal{S}} H(x ; t, \lambda) \rho(x ; t, \lambda) \mathrm{d} x$ in two different ways. From the above expression, it gives

$$
\partial_{\lambda} \int_{\mathcal{S}} H(x ; t, \lambda) \rho(x ; t, \lambda) \mathrm{d} x=\partial_{\lambda} \ell(t, \lambda)-\Omega(t, \lambda)-\lambda \partial_{\lambda} \Omega(t, \lambda)-\partial_{\lambda} \int_{\mathcal{S}} 2 x^{2} \rho(x ; t, \lambda) \mathrm{d} x .
$$

On the other hand, by Lebesgue's dominated convergence theorem, and by the symmetry in $x$ and $y$, we have

$$
\partial_{\lambda} \int_{\mathcal{S}} \int_{\mathcal{S}} \log |x-y| \rho(y ; t, \lambda) \rho(x ; t, \lambda) \mathrm{d} y \mathrm{~d} x=2 \int_{\mathcal{S}} \int_{\mathcal{S}} \log |x-y| \partial_{\lambda}(\rho(y ; t, \lambda)) \rho(x ; t, \lambda) \mathrm{d} y \mathrm{~d} x .
$$

Therefore, by differentiating (7.22) and (7.23) with respect to $\lambda$, we obtain

$$
\partial_{\lambda} \int_{\mathcal{S}} H(x ; t, \lambda) \rho(x ; t, \lambda) \mathrm{d} x=2 \int_{\mathcal{S}} \partial_{\lambda}(H(x ; t, \lambda)) \rho(x ; t, \lambda) \mathrm{d} x=2 \partial_{\lambda} \ell(t, \lambda)-2 \Omega(t, \lambda) .
$$

Putting (7.24) and (7.25) together, we obtain (7.21).

Let us consider the function

$$
F(t, \lambda)=\ell(t, \lambda)+\int_{\mathcal{S}} 2 x^{2} \rho(x ; t, \lambda) \mathrm{d} x
$$

Lemma 7.5. For $t \in(-1,1)$ and $\lambda \in\left(0, \lambda_{c}(t)\right)$, we have the following relation

$$
\Omega(t, \lambda)=\partial_{\lambda}\left[\frac{F\left(t, \lambda_{c}(t)\right)}{2}\left(\frac{\lambda}{\lambda_{c}(t)}\right)^{2}+\int_{\frac{\lambda}{\lambda_{c}(t)}}^{1} \xi F\left(t, \frac{\lambda}{\xi}\right) \mathrm{d} \xi\right] .
$$


Proof. From a direct calculation and a change of variables, the right-hand side of (7.26) is equal to

$$
\int_{\frac{\lambda}{\lambda_{c}(t)}}^{1} \xi \partial_{\lambda} F\left(t, \frac{\lambda}{\xi}\right) \mathrm{d} \xi=\left.\int_{\frac{\lambda}{\lambda_{c}(t)}}^{1} \partial_{u} F(t, u)\right|_{u=\frac{\lambda}{\xi}} \mathrm{d} \xi=\lambda \int_{\lambda}^{\lambda_{c}(t)} \frac{\partial_{u} F(t, u)}{u^{2}} \mathrm{~d} u .
$$

By using (7.21), which can be rewritten as $\partial_{\lambda} F(t, \lambda)=\Omega(t, \lambda)-\lambda \partial_{\lambda} \Omega(t, \lambda)$, the right-hand side of (7.27) becomes

$$
\lambda \int_{\lambda}^{\lambda_{c}(t)} \frac{\Omega(t, u)-u \partial_{u} \Omega(t, u)}{u^{2}} \mathrm{~d} u=\Omega(t, \lambda),
$$

where the last equality is obtained via an integration by parts, and using the identity $\Omega\left(t, \lambda_{c}(t)\right)$ $=0$.

\section{Lemma 7.6.}

$$
-\int_{0}^{\lambda_{c}(t)} \Omega(t, \lambda) \mathrm{d} \lambda=C_{1}(t)-\frac{\log 3}{2} .
$$

Proof. From Lemma 7.5, we directly obtain that

$$
-\int_{0}^{\lambda_{c}(t)} \Omega(t, \lambda) \mathrm{d} \lambda=-\frac{1}{2}\left(F\left(t, \lambda_{c}(t)\right)-F(t, 0)\right) .
$$

By (4.9) and (4.10), we obtain

$$
\begin{aligned}
F\left(t, \lambda_{c}(t)\right)= & \frac{3}{2}+2\left(\frac{4}{3} t^{2}+\frac{5}{9} t \sqrt{3+t^{2}}\right)+\frac{4 t^{3}}{27}\left(\sqrt{3+t^{2}}-t\right) \\
& +2 \log \left(2\left(t+\sqrt{3+t^{2}}\right)\right)
\end{aligned}
$$

and by (4.16), we have

$$
F(t, 0)=\frac{3}{2}+2 \log 2 .
$$

By substituting (7.30) and (7.31) into (7.29), we obtain (7.28).

\section{A Airy model RH problem}

We consider the following $\mathrm{RH}$ problem:

(a) $P_{\mathrm{Ai}}: \mathbb{C} \backslash \Sigma_{A} \rightarrow \mathbb{C}^{2 \times 2}$ is analytic, where $\Sigma_{A}$ is shown in Fig. 6 .

(b) $P_{\mathrm{Ai}}$ has the jump relations

$$
\begin{aligned}
& P_{\mathrm{Ai},+}(\zeta)=P_{\mathrm{Ai},-}(\zeta)\left(\begin{array}{cc}
0 & 1 \\
-1 & 0
\end{array}\right), \quad \text { on } \quad \mathbb{R}^{-}, \\
& P_{\mathrm{Ai},+}(\zeta)=P_{\mathrm{Ai},-}(\zeta)\left(\begin{array}{ll}
1 & 1 \\
0 & 1
\end{array}\right), \quad \text { on } \quad \mathbb{R}^{+}, \\
& P_{\mathrm{Ai},+}(\zeta)=P_{\mathrm{Ai},-}(\zeta)\left(\begin{array}{ll}
1 & 0 \\
1 & 1
\end{array}\right), \quad \text { on } \quad e^{\frac{2 \pi i}{3}} \mathbb{R}^{+} \cup e^{-\frac{2 \pi i}{3}} \mathbb{R}^{+} .
\end{aligned}
$$




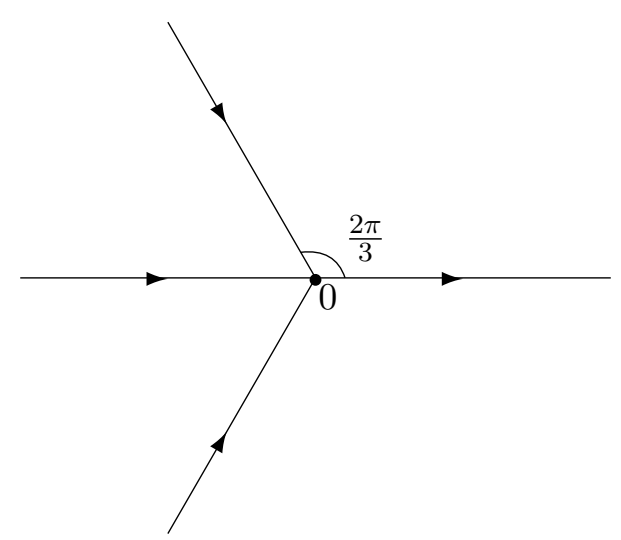

Figure 6. The jump contour $\Sigma_{A}$ for $P_{\mathrm{Ai}}(\zeta)$.

(c) As $\zeta \rightarrow \infty, z \notin \Sigma_{A}$, we have

$$
P_{\mathrm{Ai}}(\zeta)=\zeta^{-\frac{\sigma_{3}}{4}} N\left(I+\sum_{k=1}^{\infty} A_{k} \zeta^{-3 k / 2}\right) e^{-\frac{2}{3} \zeta^{3 / 2} \sigma_{3}},
$$

where $N=\frac{1}{\sqrt{2}}\left(\begin{array}{ll}1 & i \\ i & 1\end{array}\right)$ and $A_{1}=\frac{1}{8}\left(\begin{array}{cc}\frac{1}{6} & i \\ i & -\frac{1}{6}\end{array}\right)$.

This model RH problem was introduced for the first time and solved in [18], and is now wellknown. The unique solution of the above RH problem is given in terms of Airy functions, we have

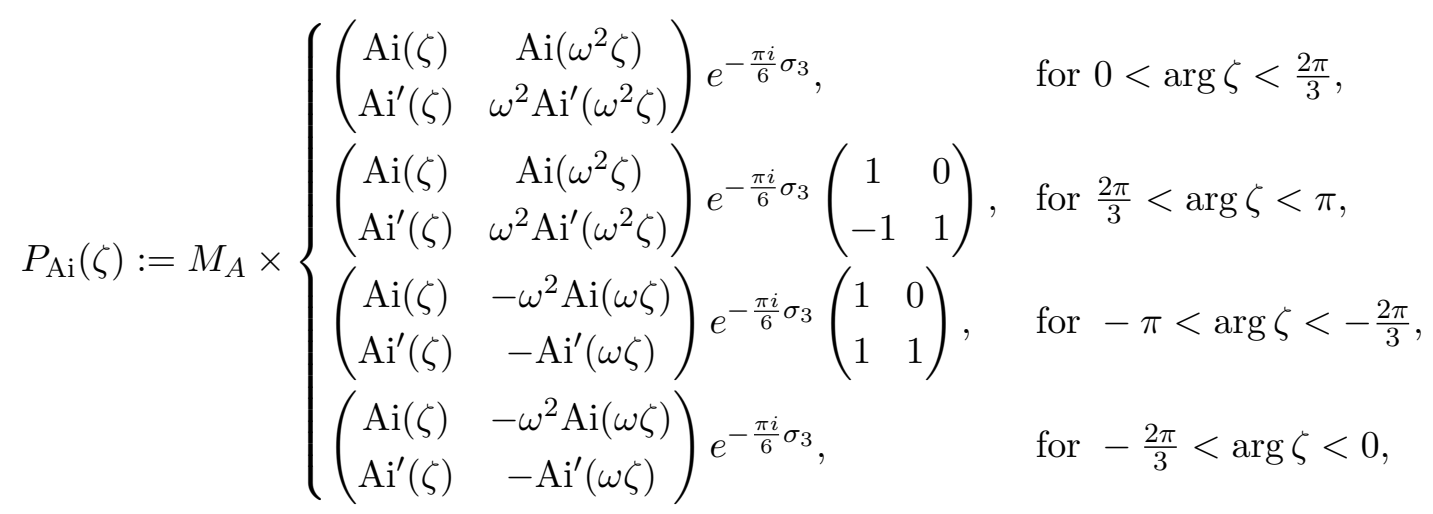

with $\omega=e^{\frac{2 \pi i}{3}}$, Ai the Airy function and

$$
M_{A}:=\sqrt{2 \pi} e^{\frac{\pi i}{6}}\left(\begin{array}{cc}
1 & 0 \\
0 & -i
\end{array}\right) .
$$

\section{B Bessel model RH problem}

We consider the following $\mathrm{RH}$ problem:

(a) $P_{\mathrm{Be}}: \mathbb{C} \backslash \Sigma_{B} \rightarrow \mathbb{C}^{2 \times 2}$ is analytic, where $\Sigma_{B}$ is shown in Fig. 7 .

(b) $P_{\mathrm{Be}}$ satisfies the jump conditions

$$
P_{\mathrm{Be},+}(\zeta)=P_{\mathrm{Be},-}(\zeta)\left(\begin{array}{cc}
0 & 1 \\
-1 & 0
\end{array}\right), \quad \zeta \in \mathbb{R}^{-}
$$




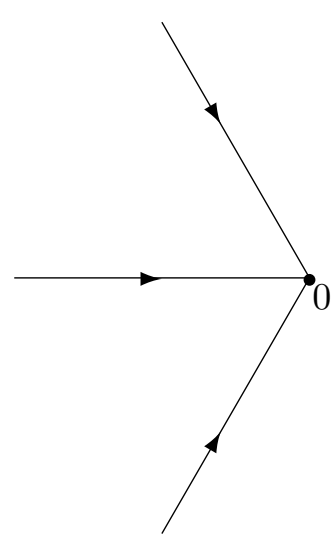

Figure 7. The jump contour $\Sigma_{B}$ for $P_{\mathrm{Be}}(\zeta)$.

$$
\begin{aligned}
& P_{\mathrm{Be},+}(\zeta)=P_{\mathrm{Be},-}(\zeta)\left(\begin{array}{cc}
1 & 0 \\
e^{\pi i \alpha} & 1
\end{array}\right), \quad \zeta \in e^{\frac{2 \pi i}{3}} \mathbb{R}^{+} \\
& P_{\mathrm{Be},+}(\zeta)=P_{\mathrm{Be},-}(\zeta)\left(\begin{array}{cc}
1 & 0 \\
e^{-\pi i \alpha} & 1
\end{array}\right), \quad \zeta \in e^{-\frac{2 \pi i}{3}} \mathbb{R}^{+}
\end{aligned}
$$

(c) As $\zeta \rightarrow \infty, \zeta \notin \Sigma_{B}$, we have

$$
P_{\mathrm{Be}}(\zeta)=\left(2 \pi \zeta^{\frac{1}{2}}\right)^{-\frac{\sigma_{3}}{2}} N\left(I+\sum_{k=1}^{\infty} B_{k} \zeta^{-k / 2}\right) e^{2 \zeta^{\frac{1}{2}} \sigma_{3}}
$$

where $N=\frac{1}{\sqrt{2}}\left(\begin{array}{cc}1 & i \\ i & 1\end{array}\right)$ and $B_{1}=\frac{1}{16}\left(\begin{array}{cc}-\left(1+4 \alpha^{2}\right) & -2 i \\ -2 i & 1+4 \alpha^{2}\end{array}\right)$.

(d) As $\zeta$ tends to 0 , the behaviour of $P_{\mathrm{Be}}(\zeta)$ is

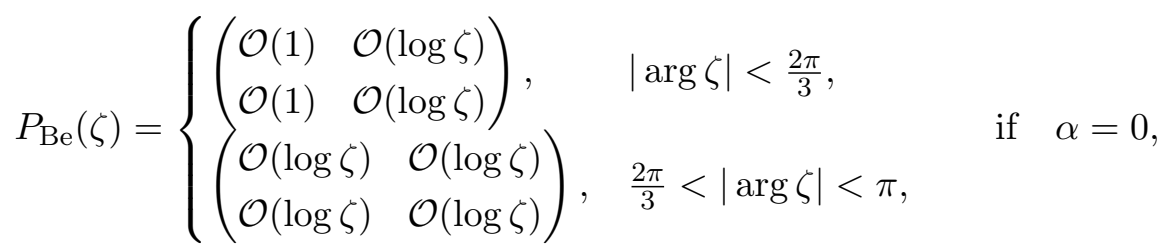

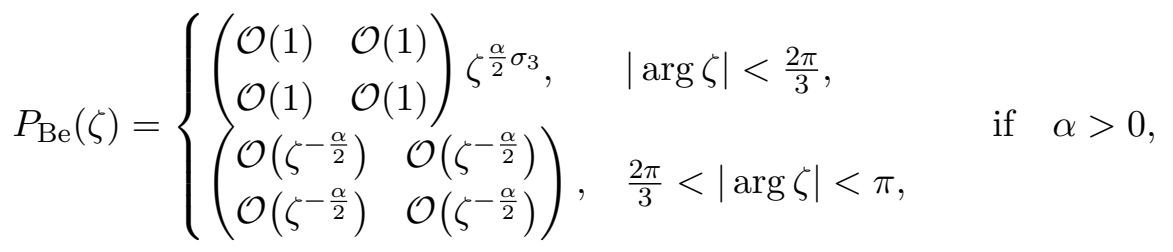

$$
\begin{aligned}
& P_{\mathrm{Be}}(\zeta)=\left(\begin{array}{ll}
\mathcal{O}\left(\zeta^{\frac{\alpha}{2}}\right) & \mathcal{O}\left(\zeta^{\frac{\alpha}{2}}\right) \\
\mathcal{O}\left(\zeta^{\frac{\alpha}{2}}\right) & \mathcal{O}\left(\zeta^{\frac{\alpha}{2}}\right)
\end{array}\right), \quad \text { if } \quad \alpha<0 .
\end{aligned}
$$

This RH problem was introduced and solved in [30]. Its unique solution is given by

$$
P_{\operatorname{Be}}(\zeta)= \begin{cases}\left(\begin{array}{cc}
I_{\alpha}\left(2 \zeta^{\frac{1}{2}}\right) & \frac{i}{\pi} K_{\alpha}\left(2 \zeta^{\frac{1}{2}}\right) \\
2 \pi i \zeta^{\frac{1}{2}} I_{\alpha}^{\prime}\left(2 \zeta^{\frac{1}{2}}\right) & -2 \zeta^{\frac{1}{2}} K_{\alpha}^{\prime}\left(2 \zeta^{\frac{1}{2}}\right)
\end{array}\right), & |\arg \zeta|<\frac{2 \pi}{3}, \\
\left(\begin{array}{cc}
\frac{1}{2} H_{\alpha}^{(1)}\left(2(-\zeta)^{\frac{1}{2}}\right) & \frac{1}{2} H_{\alpha}^{(2)}\left(2(-\zeta)^{\frac{1}{2}}\right) \\
\pi \zeta^{\frac{1}{2}}\left(H_{\alpha}^{(1)}\right)^{\prime}\left(2(-\zeta)^{\frac{1}{2}}\right) & \pi \zeta^{\frac{1}{2}}\left(H_{\alpha}^{(2)}\right)^{\prime}\left(2(-\zeta)^{\frac{1}{2}}\right)
\end{array}\right) e^{\frac{\pi i \alpha}{2} \sigma_{3}}, & \frac{2 \pi}{3}<\arg \zeta<\pi,(\mathrm{B} .4) \\
\left(\begin{array}{cc}
\frac{1}{2} H_{\alpha}^{(2)}\left(2(-\zeta)^{\frac{1}{2}}\right) & -\frac{1}{2} H_{\alpha}^{(1)}\left(2(-\zeta)^{\frac{1}{2}}\right) \\
-\pi \zeta^{\frac{1}{2}}\left(H_{\alpha}^{(2)}\right)^{\prime}\left(2(-\zeta)^{\frac{1}{2}}\right) & \pi \zeta^{\frac{1}{2}}\left(H_{\alpha}^{(1)}\right)^{\prime}\left(2(-\zeta)^{\frac{1}{2}}\right)
\end{array}\right) e^{-\frac{\pi i \alpha}{2} \sigma_{3}},-\pi<\arg \zeta<-\frac{2 \pi}{3},\end{cases}
$$


where $H_{\alpha}^{(1)}$ and $H_{\alpha}^{(2)}$ are the Hankel functions of the first and second kind, and $I_{\alpha}$ and $K_{\alpha}$ are the modified Bessel functions of the first and second kind.

\section{Acknowledgements}

C. Charlier was supported by the European Research Council under the European Union's Seventh Framework Programme (FP/2007/2013)/ ERC Grant Agreement n. 307074. A. Deaño acknowledges financial support from projects MTM2012-36732-C03-01 and MTM2015-65888C4-2-P from the Spanish Ministry of Economy and Competitivity. The authors are grateful to A.B.J. Kuijlaars for sharing a simplified proof for the first part of [11, Proposition A.1]. This inspired us to simplify the proof of Lemma 7.4. We also thank T. Claeys for a careful reading of the introduction and for useful remarks. The authors acknowledge the referees for their careful reading and useful remarks.

\section{References}

[1] Anderson G.W., Guionnet A., Zeitouni O., An introduction to random matrices, Cambridge Studies in Advanced Mathematics, Vol. 118, Cambridge University Press, Cambridge, 2010.

[2] Atkin M., Charlier C., Zohren S., On the ratio probability of the smallest eigenvalues in the Laguerre unitary ensemble, Nonlinearity 31 (2018), 1155-1196, arXiv:1611.00631.

[3] Bertola M., Bothner T., Zeros of large degree Vorob'ev-Yablonski polynomials via a Hankel determinant identity, Int. Math. Res. Not. 2015 (2015), 9330-9399, arXiv:1401.1408.

[4] Bertola M., Lee S.Y., First colonization of a hard-edge in random matrix theory, Constr. Approx. 31 (2010), 231-257, arXiv:0711.3625.

[5] Bohigas O., Pato M.P., Missing levels in correlated spectra, Phys. Lett. B 595 (2004), 171-176, nucl-th/0403006.

[6] Bothner T., Deift P., Its A., Krasovsky I., On the asymptotic behavior of a log gas in the bulk scaling limit in the presence of a varying external potential I, Comm. Math. Phys. 337 (2015), 1397-1463, arXiv:1407.2910.

[7] Brézin E., Hikami S., Characteristic polynomials of real symmetric random matrices, Comm. Math. Phys. 223 (2001), 363-382, math-ph/0103012.

[8] Buckingham R., Large-degree asymptotics of rational Painlevé-IV functions associated to generalized Hermite polynomials, arXiv:1706.09005.

[9] Charlier C., Asymptotics of Hankel determinants with a one-cut regular potential and Fisher-Hartwig singularities, Int. Math. Res. Not., to appear, arXiv:1706.03579.

[10] Charlier C., Claeys T., Asymptotics for Toeplitz determinants: perturbation of symbols with a gap, J. Math. Phys. 56 (2015), 022705, 23 pages, arXiv:1409.0435.

[11] Charlier C., Claeys T., Thinning and conditioning of the circular unitary ensemble, Random Matrices Theory Appl. 6 (2017), 1750007, 51 pages, arXiv:1604.08399.

[12] Claeys T., Birth of a cut in unitary random matrix ensembles, Int. Math. Res. Not. 2008 (2008), rnm166, 40 pages, arXiv:0711.2609.

[13] Clarkson P.A., Painlevé equations - nonlinear special functions, in Orthogonal Polynomials and Special Functions, Lecture Notes in Math., Vol. 1883, Springer, Berlin, 2006, 331-411.

[14] Clarkson P.A., Jordaan K., The relationship between semiclassical Laguerre polynomials and the fourth Painlevé equation, Constr. Approx. 39 (2014), 223-254, arXiv:1301.4134.

[15] Deaño A., Simm N.J., On the probability of positive-definiteness in the gGUE via semi-classical Laguerre polynomials, J. Approx. Theory 220 (2017), 44-59, arXiv:1610.08561.

[16] Deift P., Orthogonal polynomials and random matrices: a Riemann-Hilbert approach, Courant Lecture Notes in Mathematics, Vol. 3, New York University, Courant Institute of Mathematical Sciences, New York, Amer, Math, Soc., Providence, RI, 1999.

[17] Deift P., Its A., Krasovsky I., Asymptotics of Toeplitz, Hankel, and Toeplitz+Hankel determinants with Fisher-Hartwig singularities, Ann. of Math. 174 (2011), 1243-1299, arXiv:0905.0443. 
[18] Deift P., Kriecherbauer T., McLaughlin K.T.-R., Venakides S., Zhou X., Strong asymptotics of orthogonal polynomials with respect to exponential weights, Comm. Pure Appl. Math. 52 (1999), 1491-1552.

[19] Deift P., Kriecherbauer T., McLaughlin K.T.-R., Venakides S., Zhou X., Uniform asymptotics for polynomials orthogonal with respect to varying exponential weights and applications to universality questions in random matrix theory, Comm. Pure Appl. Math. 52 (1999), 1335-1425.

[20] Deift P., Zhou X., A steepest descent method for oscillatory Riemann-Hilbert problems, Bull. Amer. Math. Soc. (N.S.) $\mathbf{2 6}$ (1992), 119-123, math.AP/9201261.

[21] Deift P., Zhou X., A steepest descent method for oscillatory Riemann-Hilbert problems. Asymptotics for the MKdV equation, Ann. of Math. 137 (1993), 295-368.

[22] Fokas A.S., Its A.R., Kitaev A.V., The isomonodromy approach to matrix models in 2D quantum gravity, Comm. Math. Phys. 147 (1992), 395-430.

[23] Forrester P.J., Witte N.S., Application of the $\tau$-function theory of Painlevé equations to random matrices: PIV, PII and the GUE, Comm. Math. Phys. 219 (2001), 357-398, math-ph/0103025.

[24] Foulquié Moreno A., Martínez-Finkelshtein A., Sousa V.L., On a conjecture of A. Magnus concerning the asymptotic behavior of the recurrence coefficients of the generalized Jacobi polynomials, J. Approx. Theory 162 (2010), 807-831, arXiv:0905.2753.

[25] Garoni T.M., On the asymptotics of some large Hankel determinants generated by Fisher-Hartwig symbols defined on the real line, J. Math. Phys. 46 (2005), 043516, 19 pages, math-ph/0411019.

[26] Gromak V.I., Laine I., Shimomura S., Painlevé differential equations in the complex plane, De Gruyter Studies in Mathematics, Vol. 28, Walter de Gruyter \& Co., Berlin, 2002.

[27] Its A., Krasovsky I., Hankel determinant and orthogonal polynomials for the Gaussian weight with a jump, in Integrable systems and random matrices, Contemp. Math., Vol. 458, Amer. Math. Soc., Providence, RI, 2008, 215-247, arXiv:0706.3192.

[28] Kajiwara K., Ohta Y., Determinant structure of the rational solutions for the Painlevé IV equation, J. Phys. A: Math. Gen. 31 (1998), 2431-2446, solv-int/9709011.

[29] Krasovsky I., Correlations of the characteristic polynomials in the Gaussian unitary ensemble or a singular Hankel determinant, Duke Math. J. 139 (2007), 581-619, math-ph/0411016.

[30] Kuijlaars A.B.J., McLaughlin K.T.-R., Van Assche W., Vanlessen M., The Riemann-Hilbert approach to strong asymptotics for orthogonal polynomials on $[-1,1]$, Adv. Math. 188 (2004), 337-398, math.CA/0111252.

[31] Kuijlaars A.B.J., Vanlessen M., Universality for eigenvalue correlations at the origin of the spectrum, Comm. Math. Phys. 243 (2003), 163-191, math-ph/0305044.

[32] Mehta M.L., Random matrices, Pure and Applied Mathematics (Amsterdam), Vol. 142, 3rd ed., Elsevier/Academic Press, Amsterdam, 2004.

[33] Mehta M.L., Normand J.-M., Probability density of the determinant of a random Hermitian matrix, J. Phys. A: Math. Gen. 31 (1998), 5377-5391.

[34] Mo M.Y., The Riemann-Hilbert approach to double scaling limit of random matrix eigenvalues near the "birth of a cut" transition, Int. Math. Res. Not. 2008 (2008), rnn042, 51 pages, arXiv:0711.3208.

[35] Okamoto K., Studies on the Painlevé equations. III. Second and fourth Painlevé equations, $P_{\mathrm{II}}$ and $P_{\mathrm{IV}}$, Math. Ann. 275 (1986), 221-255.

[36] Olver F.W.J., Olde Daalhuis A.B., Lozier D.W., Schneider B.I., Boisvert R.F., Clark C.W., Miller B.R., Saunders B.V. (Editors), NIST digital library of mathematical functions, Release 1.0.13 of 2016-09-16, available at http://dlmf.nist.gov/.

[37] Saff E.B., Totik V., Logarithmic potentials with external fields, Grundlehren der Mathematischen Wissenschaften, Vol. 316, Springer-Verlag, Berlin, 1997.

[38] Szegő G., Orthogonal polynomials, American Mathematical Society, Colloquium Publications, Vol. 23, 4th ed., Amer. Math. Soc., Providence, R.I., 1975.

[39] Vanlessen M., Strong asymptotics of Laguerre-type orthogonal polynomials and applications in random matrix theory, Constr. Approx. 25 (2007), 125-175, math.CA/0504604.

[40] Winternitz P., Physical applications of Painlevé type equations quadratic in the highest derivatives, in Painlevé Transcendents (Sainte-Adèle, PQ, 1990), NATO Adv. Sci. Inst. Ser. B Phys., Vol. 278, Plenum, New York, 1992, 425-431.

[41] Wu X.-B., Xu S.-X., Zhao Y.-Q., Gaussian unitary ensemble with boundary spectrum singularity and $\sigma$-form of the Painlevé II equation, Stud. Appl. Math. 140 (2018), 221-251, arXiv:1706.03174. 